\title{
WestVirginiaUniversity
}

THE RESEARCH REPOSITORY @ WVU

Graduate Theses, Dissertations, and Problem Reports

2015

\section{Plasmonic Nanostructures for Biosensor Applications}

\author{
Akshitha Gadde
}

Follow this and additional works at: https://researchrepository.wvu.edu/etd

\section{Recommended Citation}

Gadde, Akshitha, "Plasmonic Nanostructures for Biosensor Applications" (2015). Graduate Theses, Dissertations, and Problem Reports. 7088.

https://researchrepository.wvu.edu/etd/7088

This Thesis is protected by copyright and/or related rights. It has been brought to you by the The Research Repository @ WVU with permission from the rights-holder(s). You are free to use this Thesis in any way that is permitted by the copyright and related rights legislation that applies to your use. For other uses you must obtain permission from the rights-holder(s) directly, unless additional rights are indicated by a Creative Commons license in the record and/ or on the work itself. This Thesis has been accepted for inclusion in WVU Graduate Theses, Dissertations, and Problem Reports collection by an authorized administrator of The Research Repository @ WVU. For more information, please contact researchrepository@mail.wvu.edu. 


\title{
Plasmonic Nanostructures for Biosensor Applications
}

\author{
Akshitha Gadde \\ Thesis submitted to the \\ Benjamin M. Statler College of Engineering and Mineral Resources \\ at West Virginia University \\ in partial fulfillment of the requirements \\ for the degree of \\ Masters in Science \\ in \\ Electrical Engineering \\ Jeremy M. Dawson, Ph.D., Chair \\ Lawrence A. Hornak, Ph.D. \\ Dimitris Korakakis, Ph.D.
}

Lane Department of Computer Science and Electrical Engineering

Morgantown, West Virginia

2015

Keywords: Plasmonics, Labeled detection, Gold, Metal Enhanced Fluorescence, Nanofabrication

Copyright 2015 Akshitha Gadde 


\title{
ABSTRACT \\ Plasmonic Nanostructures for Biosensor Applications \\ Akshitha Gadde
}

\begin{abstract}
Improving the sensitivity of existing biosensors is an active research topic that cuts across several disciplines, including engineering and biology. Optical biosensors are the one of the most diverse class of biosensors which can be broadly categorized into two types based on the detection scheme: label-based and label-free detection. In label-based detection, the target bio-molecules are labeled with dyes or tags that fluoresce upon excitation, indicating the presence of target molecules. Label-based detection is highly-sensitive, capable of single molecule detection depending on the detector type used. One method of improving the sensitivity of label-based fluorescence detection is by enhancement of the emission of the labels by coupling them with metal nanostructures. This approach is referred as plasmon-enhanced fluorescence (PEF). PEF is achieved by increasing the electric field around the nano metal structures through plasmonics. This increased electric field improves the enhancement from the fluorophores which in turn improves the photon emission from the fluorophores which, in turn, improves the limit of detection. Biosensors taking advantage of the plasmonic properties of metal films and nanostructures have emerged an alternative, low-cost, high sensitivity method for detecting labeled DNA. Localized surface plasmon resonance (LSPR) sensors employing noble metal nanostructures have recently attracted considerable attention as a new class of plasmonic nanosensors.

In this work, the design, fabrication and characterization of plasmonic nanostructures is carried out. Finite difference time domain (FDTD) simulations were performed using software from Lumerical Inc. to design a novel LSPR structure that exhibit resonance overlapping with the absorption and emission wavelengths of quantum dots (QD). Simulations of a composite $\mathrm{Au} / \mathrm{SiO} 2$ nanopillars on silicon substrate were performed using FDTD software to show peak plasmonic enhancement at QD emission wavelength (560nm). A multi-step fabrication process was developed to create plasmonic nanostructures, and the optical characterization of emission enhancement was performed.
\end{abstract}




\section{ACKNOWLEDGMENTS}

First and foremost, I would like to express my deepest gratitude to my advisor Dr. Jeremy Dawson for providing me an opportunity for research in semiconductors and for his constant encouragement and guidance throughout my masters. I was able to come this point only because of his guidance, assistance, encouragement and funding me throughout the period of my research. I would also like to thank Dr. Hornak, and Dr. Korakakis for accepting to serve on my committee.

This thesis is dedicated to my dear parents and sister, Venkateswara Rao, Indumathi and Arpitha without whom I could have never reached this point in my life. Thank you for your love, support, and sacrifices!

I would like to thank my lab mates Anand Kadiyala, Kyle smith, Sujan Kasani, Chloe Snyder, Rupa, Yassar Khouj, Christo Robinson, and Seth Leffel for their support.

I would like to thank my friends Ravindra Gadde, Krishna Teja, Usha Geddam, Sonali Moon, Saurabh Chaudhari , Chanukya Gadde, Siddhartha Gadiraju, Priyanka Reddy, Aditya, Anveeksh, Savan Suri, Maurya Srungarapu, Harika Bobba, Sneha, Raghu, Aarthi, Prathyusha who supported me and made my life is USA beautiful.

Finally, I would like to thank WVU SRF staff for their assistance. 


\section{$\underline{\text { Table of Contents }}$}

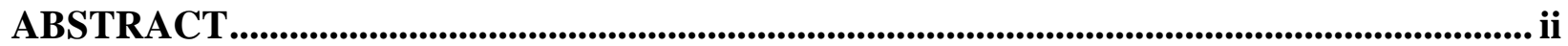

Plasmonic Nanostructures for Biosensor Applications ................................................................... ii

Akshitha Gadde....................................................................................................................................... ii

ACKNOWLEDGMENTS .................................................................................................................... iii

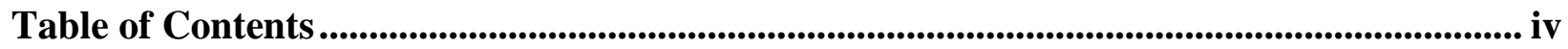

Table of Figures .............................................................................................................................................. viii

List of Tables ............................................................................................................................................ xi

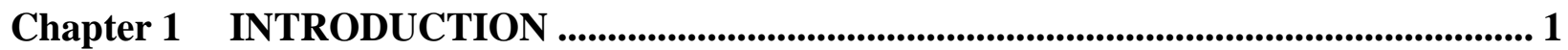

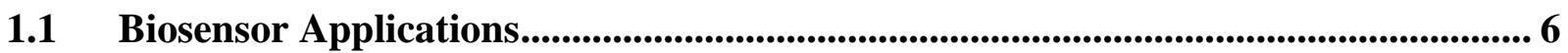

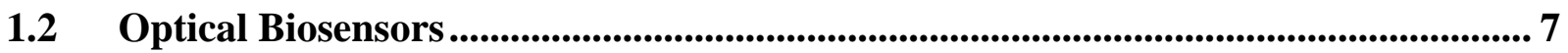

1.2.1 Label free biosensors ........................................................................................................ 7

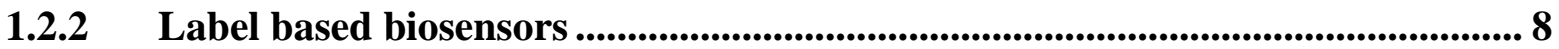

1.2.3 Fluorescence - based biosensors .......................................................................... 8

1.3 Photonic Crystals................................................................................................................. 9 
1.3.1 Photonic Crystal Biosensors......................................................................................... 10

1.4 Fluorescence Enhancement comparison ........................................................................... 11

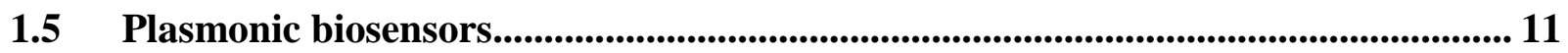

1.5.1 Metal Enhanced Fluorescence ........................................................................... 12

1.5.2 Applications of Metal Enhanced Fluorescence ........................................................ 13

1.6 Scope of this thesis....................................................................................................................... 17

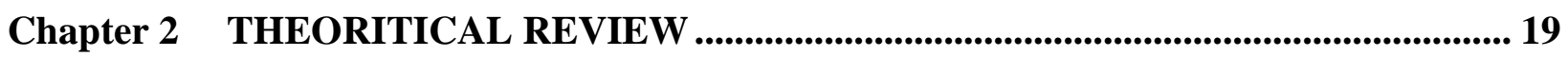

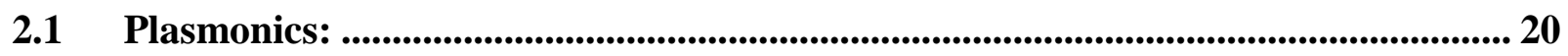

2.1.1 Surface Plasmons ............................................................................................. 20

2.1.2 Localized Surface Plasmon Resonance (LSPR) ..................................................... 21

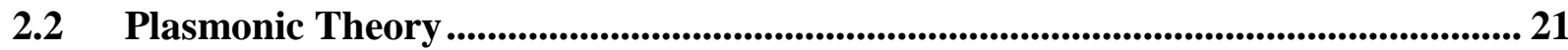

2.3 Mie theory ….............................................................................................................................. 22

2.3.1 The Radiating Dipole....................................................................................................... 22

2.4 Extinction power ..................................................................................................................... 25

$2.5 \quad$ Fluorescence Spectroscopy .................................................................................................. 25

2.5.1 Photophysics of fluorophore .......................................................................................... 26

2.6 Enhancement of Fluorescence............................................................................................ 28

2.6.1 Plasmon- Dye Interactions ............................................................................................ 28

2.6.2 Energy Transfer ................................................................................................................. 29 
2.6.3 Electric Field effects......................................................................................... 30

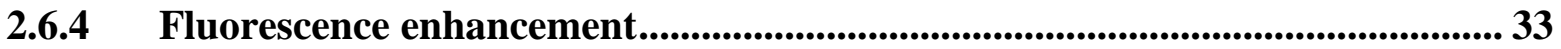

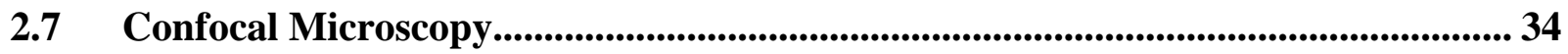

2.8 Concentration calculation for Quantum dots: .......................................................... 35

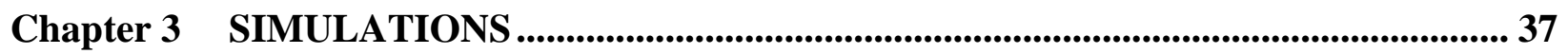

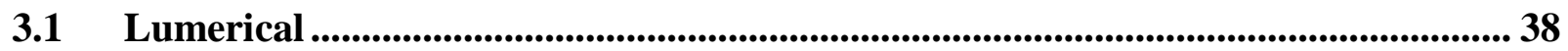

3.2 Localized surface plasmon resonance (LSPR) Design Parameters: ......................... 39

3.2.1 LSPR Design Parameters, Material Effect .................................................... 40

3.3 LSPR Design Parameters, Size Effect ............................................................. 41

3.3.1 LSPR design parameters, effect of refractive index .................................... 41

3.3.2 LSPR design parameters, spacing effect................................................... 42

3.3.3 LSPR Design Parameters, thickness of gold deposition ................................ 45

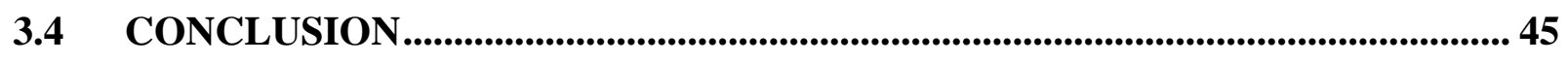

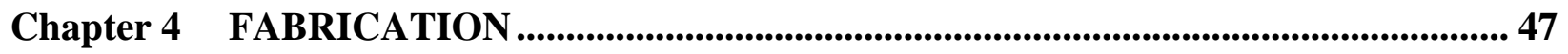

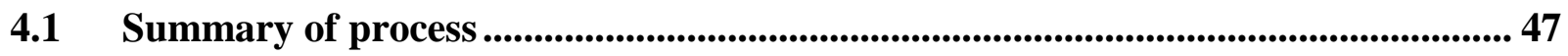

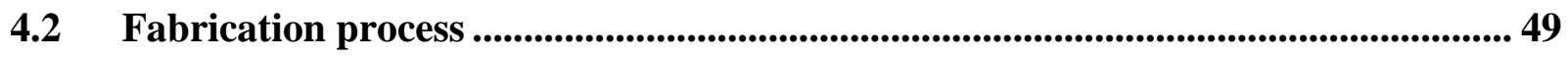

4.2.1 Sample Cleaning.............................................................................................. 50

4.3 Nanopillar Fabrication ................................................................................................ 50

4.3.2 Electron beam Lithography ................................................................................ 51 


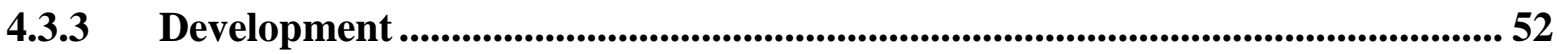

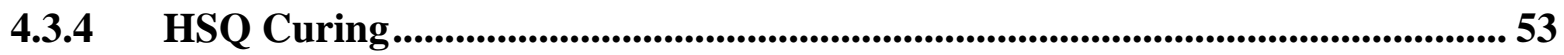

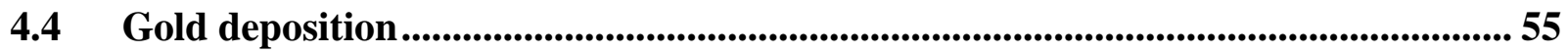

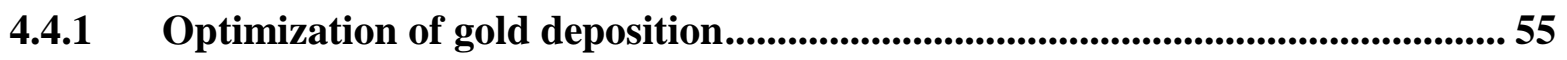

4.5 Spacer deposition..................................................................................................... 59

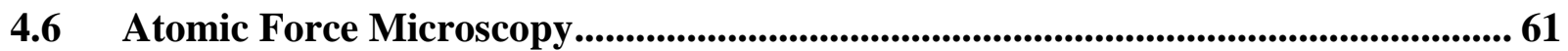

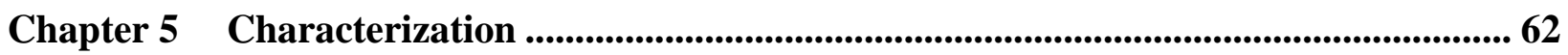

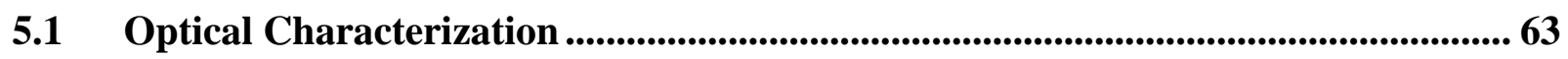

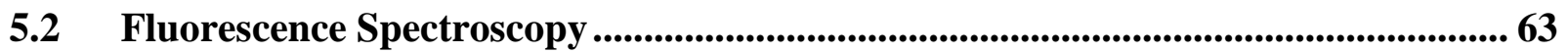

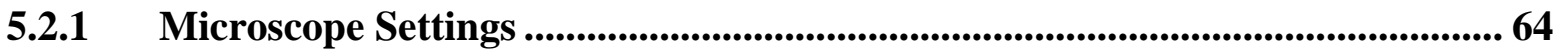

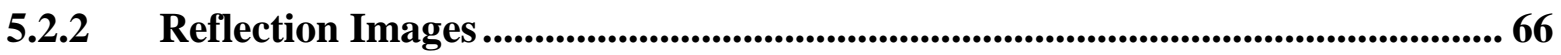

5.2.3 Fluorescent Microscope Images.............................................................. 67

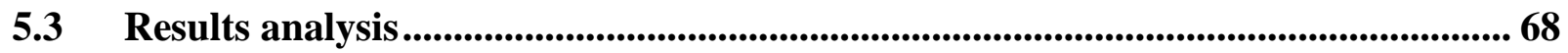

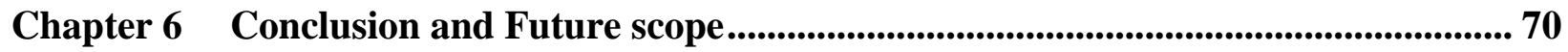

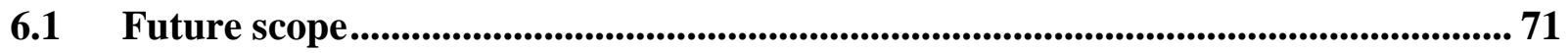

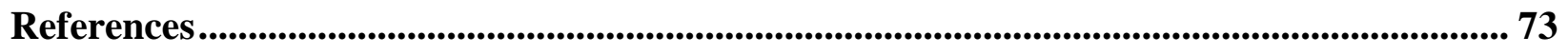

APPENDIX A: PREPROCESS PREPARATION ........................................................ 84

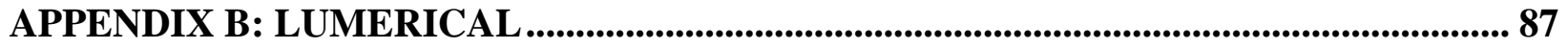

APPENDIX C: MATLAB program for image analysis ............................................................ 94 


\section{TABLE OF FIGURES}

FIGURE 1-1 ILLUSTRATION OF TYPICAL DISEASE DIAGNOSIS PIPELINE, WHERE SAMPLES MAY NEED TO BE SENT TO SPECIALIZED MEDICAL FACILITIES, RESULTING IN LONGER WAIT TIMES (COURTESY: GOOGLE IMAGES) 1

FIGURE 1-2: GLUCOSE BIOSENSOR UTILIZING A SPECK OF BLOOD FOR TEST [75] .3

FIGURE 1-3: CMOS BASED IMPLANTABLE GLUCOSE BIOSENSOR [4] .4

FIGURE 1-4: FLUORESCENCE EMISSION ENHANCEMENT FROM THE FLUOROPHORES ON TOP OF (A) A NORMAL SUBSTRATE (B) A PHOTONIC CRYSTAL (C) PLASMONIC LATTICE STRUCTURE

FIGURE 1-5: PHOTOGRAPH OF FLUORESCEIN-LABELED HSA ON QUARTZ (LEFT) AND ON SIFS (RIGHT) AS OBSERVED WITH LASER EXCITATION OF 430 NM EXCITATION LONG PASS EMISSION FILER AT 480 NM. THE EXCITATION WAS PROGRESSIVELY MOVED FROM THE QUARTZ SIDE TO THE

FIGURE 1-6: (A) EMISSION SPECTRA OF INDOCYANINE GREEN-ALBUMIN (ICG-HSA) BOUND TO UNSILVERED QUARTZ SLIDES OR SILVER ISLAND FILMS. (B) PHOTO STABILITY OF ICG-HSA ON QUARTZ AND ON SIFS .

FIGURE 1-7: EMISSION SPECTRA OF SS DNA BOUND (SOLID LINE) TO SILVER NANOPARTICLES AND IN SOLUTION (DASHED LINE).

FIGURE 1-8:ENHANCEMENT FACTOR OF 30FOLD ON THE GOLD NANOPILLARS COMPARE TO THE BACKGROUND (SILICON SUBSTRATE)

FIGURE 1-9: ENHANCEMENT OF THE QUANTUM DOTS ON THE GOLD NANOPILLARS COMPARED WITH THE SILICON SUBSTRATE

FIGURE 1-10: (A) CY5 DYE LABELED DNA SPIN COAETED ON GLASS (B) CY5 DYE LABELED DNA TETHERED TO SIFS (C) CY5 DYE LABELED DNA COUPLED TO SILVER NANOPARTICLES 
FIGURE 2-1: INDUCED DIPOLE MOMENT IN A METAL PARTICLE WITH AN EXTERNAL ELECTROMAGNETIC FIELD

FIGURE 2-2: PROCESS OF FLUORESCENCE 26

FIGURE 2-3: INTERACTION OF FLUOROPHORE WITH THE ELECTRIC FIELD GENERATED BY GOLD NANOPARTICLE.

FIGURE 2-4: SPECTRUM OF CDSE/ ZNS QUANTUM DOTS .35

FIGURE 3-1: SIMULATION SPACE FOR PLASMONIC NANOSTRUCTURE SIMULATION 37

FIGURE 3-2: ELECTRIC FIELD INTENSITY ENHANCEMENT AROUND THE NANOSTRUCTURE . .39

FIGURE 3-3: EXTINCTION PEAK VARIATION WITH REFRACTIVE INDEX. 41

FIGURE 3-4: ELECTRIC FIELD INTENSITY INDICATING HOTSPOTS IN BETWEEN THE GOLD NANOPILLARS. 42

FIGURE 3-5: EXTINCTION PEAK VARIATION THIN GOLD FILM VS PATTERNED GOLD FILM . .43

FIGURE 3-6: EXTINCTION PEAK VARIATION WITH DIAMETER OF THE NANOPARTICLES....................44

FIGURE 3-7: EXTINCTION PEAK VARIATION WITH GOLD DEPOSITION THICKNESS . .45

FIGURE 4-1: FABRICATED SQUARE PLASMONIC GOLD NANOPILLARS ON SILICON SUBSTRATE.....49 FIGURE 4-2: SIDE VIEW OF THE FABRICATED PLASMONIC NANOSTRUCTURE. .50

FIGURE 4-3: FABRICATED SQUARE NANOSTRUCTURES WITH VARYING DOSAGES .52

FIGURE 4-4: SEM IMAGE OF SQUARE NANOSTRUCTURES AT MAGNIFICATION OF 90,000X. . .54

FIGURE 4-5: FABRICATED SQUARE NANOSTRUCTURES ON SILICON SUBSTRATE USING E-BEAM LITHOGRAPHY .54

FIGURE 4-6: DESIGNED PARAMETERS FOR GOLD DEPOSITION ON TOP OF NANOPILLARS . .55

FIGURE 4-7: SEM PICTURE SHOWING GOLD DEPOSITION WITH DEPOSITION RATE OF 2.0 A/S ..... . .56

FIGURE 4-8: MEASURED VALUES OF GOLD DEPOSITION THICKNESS WITH DEPOSITION RATE OF $2.0 \mathrm{~A} / \mathrm{S}$ . .56 
FIGURE 4-9: SEM IMAGE OF GOLD DEPOSITION ON TOP OF $\mathrm{SIO}_{2} \mathrm{SQUARE}_{\text {NANOPILLARS }}$

FIGURE 4-10:SEM IMAGE OF FABRICATED STRUCTURE AT AN ANGLE OF $70^{\circ}$ .58

FIGURE 4-11: OPTIMIZED GOLD DEPOSITION ON TOP OF SQUARE NANOPILLARS WITH DEPOSITION

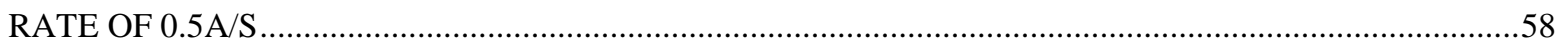

FIGURE 4-12:SEM IMAGE OF FABRICATED STRUCTURE AT AN ANGLE OF 30DEG ............................59

FIGURE 4-13:SEM IMAGE SHOWING THICKNESS OF GOLD DEPOSITION AT AN ANGLE OF 90 DEG ...60

FIGURE 4-14:SEM PICTURE SHOWING SQUARE NANOPILLARS AFTER DEPOSITION OF SIO $2 \ldots \ldots \ldots \ldots . . .60$

FIGURE 4-15:AFM IMAGE $(5 \mu \mathrm{M}$ X 5$\mu \mathrm{M})$ SIO2 DEPOSITED SI SUBSTRATE WHICH FORMED AS ISLANDS .61

FIGURE 5-1: OPTICAL IMAGE OF THE FABRICATED NANOSTRUCTURES WITH VARYING DOSAGES

FIGURE 5-2: BLOCK DIAGRAM OF MICROSCOPE .64

FIGURE 5-3: SPECTRUM OF CDSE/ZNS QUANTUM DOTS .65

FIGURE 5-4: REFLECTION IMAGE OF THE SAMPLE FOCUSED WITH A LASER .66

FIGURE 5-5: FLUORESCENT MICROSCOPE EXCITED WITH A LASER SOURCE. 67 


\section{List of Tables}

TABLE 3-1: TABLE SHOWING THE MODELED PARAMETER AND THE TOLERANCE FOR THE

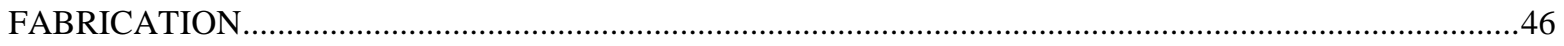

TABLE 4-1: SUMMARY OF THE PROCESS: FABRICATION OF PLASMONIC NANOPILLARS ...................47

TABLE 5-1:COMPARING PIXEL VALUES ON (LEFT) PLASMONIC NANOSTRUCTURE AND (RIGHT)

THIN GOLD FILM 


\section{Chapter 1}

\section{INTRODUCTION}

The development of nanoscale optical biosensor that enables real-time detection of target molecules with high sensitivity and selectivity is an active research topic that applies to many fields. The development of low-cost, portable and high-throughput sensing technologies that can enable point of care diagnostics and monitor various medical conditions are crucial for forensics, drug discovery, disease screening, and early detection of diseases.

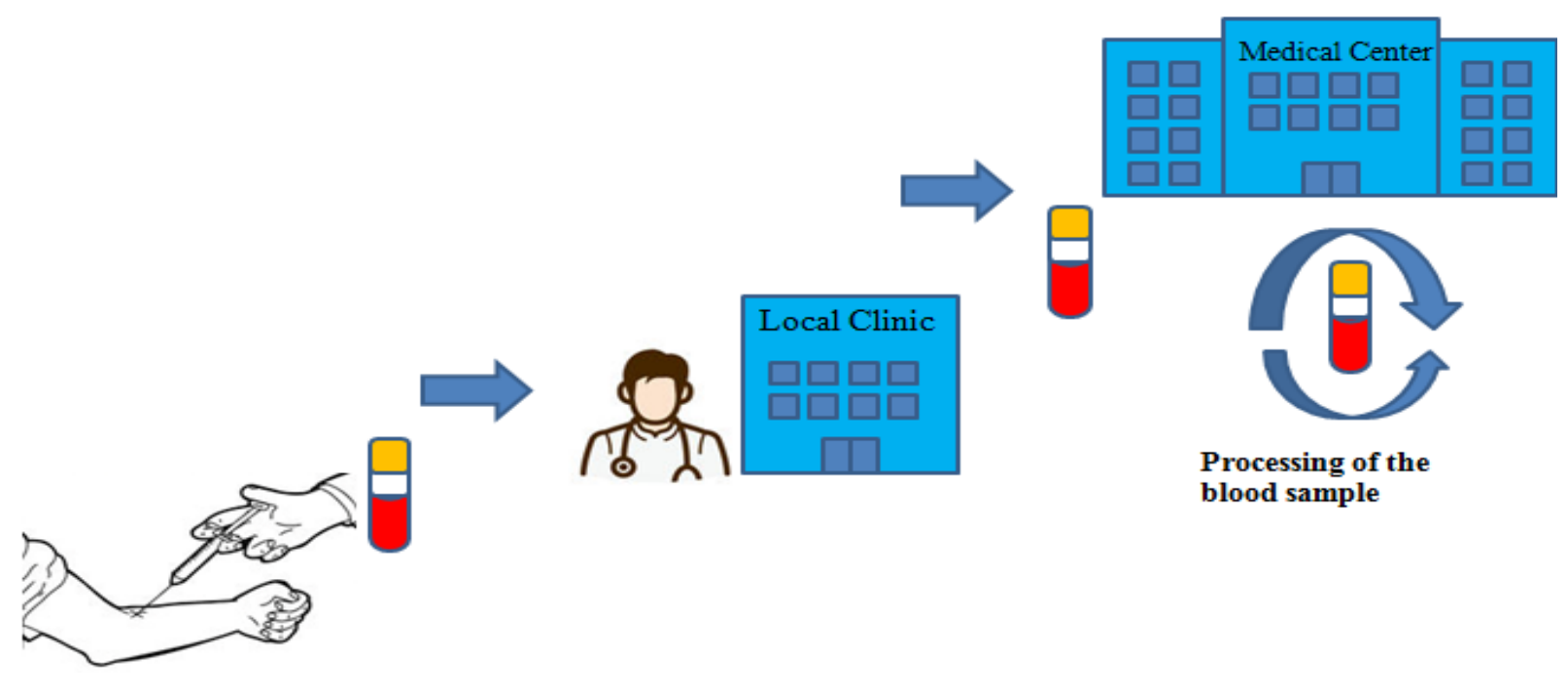

Blood from patient

Figure 1-1 Illustration of typical disease diagnosis pipeline, where samples may need to be sent to specialized medical facilities, resulting in longer wait times (Courtesy: Google Images). 
Typical process flow for the diagnosis of a disease is illustrated in Figure 1.1. Traditional diagnostic technologies are often time-consuming and require complex, costly laboratory procedures, trained laboratory technicians, and advanced bulky medical instrumentation. For example, considering traditional method for detection of diabetes, blood glucose test strips were used, where reagent pad is coated with a drop of blood and the change in color signifies the glucose level in the blood. The reagent pad is a reaction system which includes glucose oxidation, peroxidase, and chromogen system which develops color upon the oxidation of glucose present in the blood. The drop of blood is placed on the reagent, where the glucose in the blood is oxidized and it passes through the semi-permeable membrane and reacts with chromogen to give blue color. It takes a minute for reaction to occur and then it's matched with series of color blocks. But under normal lighting conditions it's hard to visualize the color blocks and variation in visual acuity of each individual. It requires perfect lighting conditions to obtain precise and accurate readings. This procedure requires a physician, laboratory and technician [1].

Biosensor technologies show promise in overcoming problems mentioned above. For example, glucose biosensor technology has been developed to supplement and replace reagent-based detection, which uses an enzyme electrode strip. It involves of oxidation of glucose in blood. The current is measured during the electro chemical reaction, which can be detected by amperometric sensor [2]. These require $0.3-1 \mu \mathrm{L}$ of blood and 10sec for result. The magnitude of the current is correlated to the concentration of glucose. This provides accurate identification in a portable and easy to use diagnostic mechanism. It doesn't require perfect lighting conditions as the amount of sugar is shown in the form of magnitude of current, which can be easily understood. Biosensor technologies have several advantages over conventional diagnostic analysis techniques. They include low cost and manufacturing and operation, as well as field portability [3]. Various technologies have been used and wide variety of different biosensors have been developed. Now the research focus is improving the sensitivity of these biosensors. 


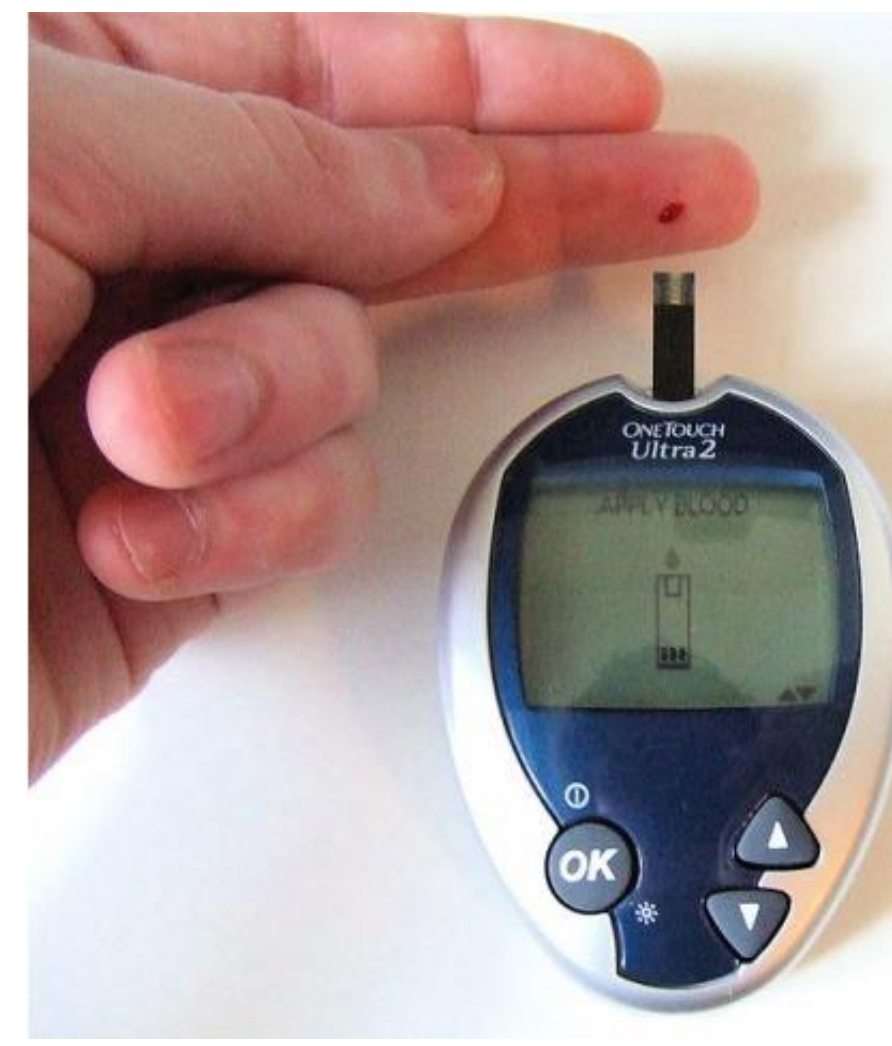

Figure 1-2: Glucose biosensor utilizing a speck of blood for test [75]

Advancements in biosensor research led to the discovery of continuous glucose monitoring system with an inbuilt sensor chip. An implantable glucose sensor with CMOS image sensor based chip which can be implanted into the body for the continuous monitoring of the blood sugar has been developed. This technology used an optical sensor system with a fluorescent hydrogel which is glucose responsive. As the glucose level in the blood increases, the fluorescence intensity from the dye increases [4]. LED is used to excite the fluorophores to obtain the fluorescence signal collected by the CMOS image sensor. This is experimentally verified by in-vivo experiments. There is continuous need for the improvement in sensitivity. 


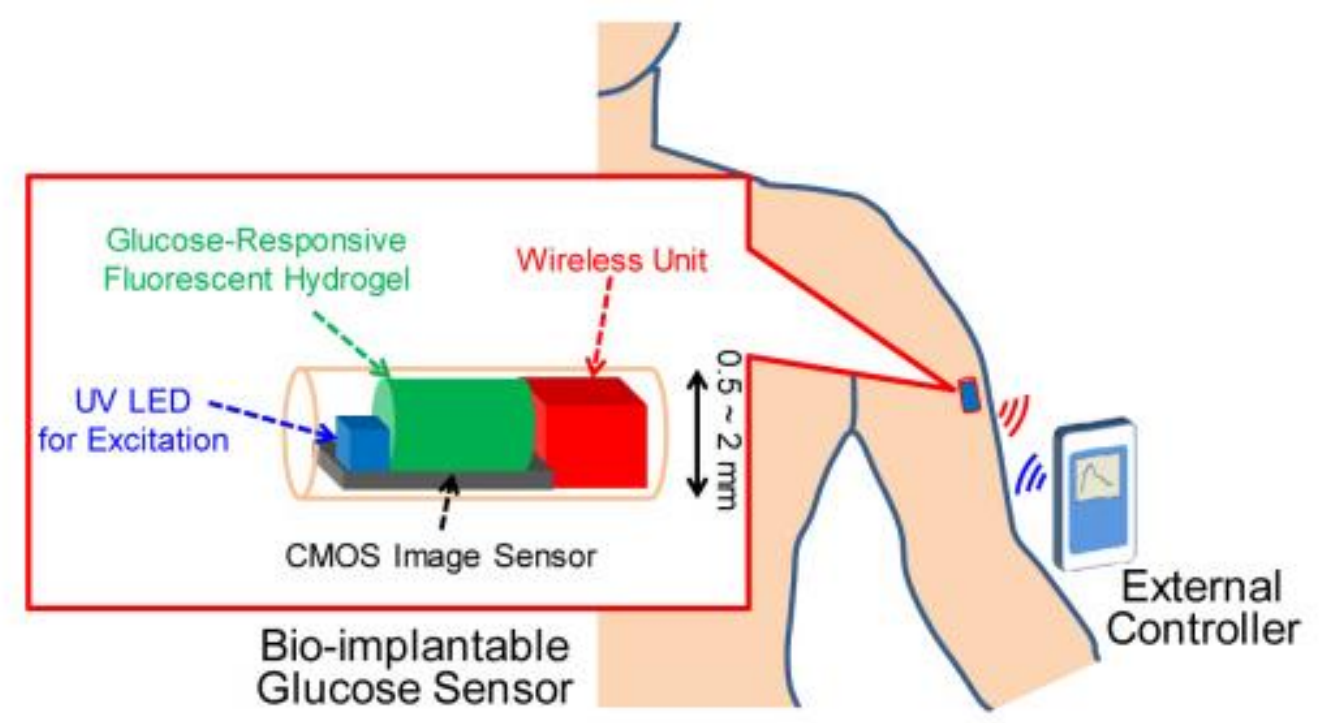

Figure 1-3: CMOS based implantable glucose biosensor [4]

The research of Interdisciplinary Research Team (IRT1) of the Nanotechnology Sensing Advances in Field and Environment (NanoSAFE) program in West Virginia University focuses on developing cost effective, portable biosensors for rapid detection of toxins and pathogens, with specific interest on DNA, heavy metals, and small molecule toxins [5]. The goal of IRT1 is creating a portable, high throughput, rapid DNA identification platform via collaborations within microbiology, pharmacy and chemistry disciplines to improve the sensitivity and selectivity of DNA-based detection methods. The isolation of various strains of pathogens is performed by Dr. Lumkomski's group (Microbiology) at WVU Health Sciences. This isolation method results in the attachment of a fluorescent dye to the polymerase chain reaction (PCR) product. Nanocapillary separation of the PCR products in a novel phospholipid gel is performed by Dr. Holland's group (Chemistry). Then separated PCR products attached with a dye flow onto the sensor designed and fabricated by Dr. Dawson's group through the microfluidic channels designed by Dr. Holland's group.

The capability of imaging and detecting labeled DNA molecules is critical in this study. Originally, photonic crystal device was employed for detection of DNA to take advantage of localization and concentration of fluorophore photon emission. However, plasmonic techniques 
that show promise for greater levels of emission enhancement due to the potential for higher levels of sensitivity that can be gained through higher levels of enhancement.

Detection of DNA is often performed by labeling DNA with a fluorescent dye, or fluorophore. Fluorophores absorb light at one particular wavelength and emit light in a different wavelength, typically longer wavelength. When employed in biosensor technologies, this kind of detection involving a label is often called label-based detection. For photonic crystal-based detection schemes, an excitation source is used to excite the fluorophore and the emission wavelength of the fluorophore is in the photonic bandgap. Within the photonic bandgap, the electric field energy becomes more concentrated in the material with high dielectric constant. By taking advantage of optical bandgap, the fluorescent tagged targets emit more light in comparison with an ordinary surface occurs by the detected by the reflecting mechanism of photonic crystals by taking advantage of optical bandgap [6,7]. Until now, using photonic crystals, fluorescence experiments were performed by considering optical energy which is radiated into far field which is several wavelengths from the fluorophore, but detection is usually done at macroscopic distances from the sample. However, there is a rapidly growing demand for increasing sensitivity to detect trace amounts of analytes with advancements in fluorescence based technologies. One prominent way to improve sensitivity of label-based detection schemes is by enhancement of signal from fluorophore labels by coupling them with metal nanostructures [8]. This approach is referred as Metal-enhance fluorescence (MEF)/ plasmon-enhanced fluorescence (PEF) [9]. Metal nanostructures alter the near field around them by electron oscillations. Near-field interactions which occur within only $200 \mathrm{~nm}$ distance of the metal nanoparticle [10]. These near-field interactions alter the spectral properties of fluorophore by interaction of fluorophore with the electron cloud present on the metal. These interactions improve the fluorescence signal by changing the emission. This technique has an advantage of: Improving the excitation rate of the fluorophore at its absorption wavelength $\left(\lambda_{\mathrm{ab}}\right)$ and it enhances the efficiency of collecting fluorescence signal at emission wavelength $\left(\lambda_{\mathrm{em}}\right)$ by using highly coupled plasmon coupled emission [9]. In addition, metal nanostructures can create unique fluorophores with high quantum yield and shorter lifetimes. This thesis explores the plasmonic phenomenon and fluorescence enhancement using plasmonics for sensitive bio-sensing. This effort included the following tasks: 
- Device Modeling and Design: Plasmonic nanostructures ware designed using Finite difference time domain (FDTD) software tool from Lumerical Inc. Critical dimensions were extracted for device fabrication.

- Fabrication: A fabrication process was developed to realize the optimal of plasmonic nanostructures. This multistep process involved application of electron beam (e-beam) lithography, thin film deposition of metals and dielectrics using physical vapor deposition technique, and optical lithography.

- Characterization: Characterization of plasmonic nanostructures is performed using various instruments: Scanning electron microscopy, Atomic force microscopy, Optical microscope, and Fluorescence microscope.

\subsection{Biosensor Applications}

The development of highly sensitive and selective biosensors is an active research topic that cuts across many disciplines and application areas. Research in the field of biosensors originated in 1960s [11]. The term biosensor is an acronym for "biological sensor", and refers to compact analytical devices that are used for detection of biological elements such as single-stranded DNA, enzymes, and receptors. Biosensors combine biological elements with transducers and detectors that can transform the signal resulting from the interaction of an analyte with a biological element into a measurable optical or electrical signal [11]. A transducer is typically physicochemical transducer that produces a physicochemical change upon detection. Physicochemical transducers can be optical, electronic, electrochemical, gravimetric and piezoelectric. Based on the type of bio-transducer, biosensors can be classified as optical biosensors, electrochemical biosensors, electronic biosensors, gravimetric biosensors and piezoelectric biosensors.

Optical biosensors are most popular in analytical sensor field. This is because they can read and generate the signal rapidly, and have an advantage of applying Visible/ Ultra Violet (UV)/ Infrared (IR) optical energy as a source for detection compared with other transducers. Optical biosensors have capability of providing multiplexed detection and are immune to electromagnetic interference [12]. 


\subsection{Optical Biosensors}

Optical biosensors are diverse class of biosensors, as they can be used for many different types of spectroscopy measurements, such as, fluorescence, phosphorescence, absorption, raman, refraction, and SERS [13, 14, 15, 16, 17, 18, 19]. Most commonly optical biosensors utilize light as source for excitation. Optical biosensors are designed to operate in UV/ Visible/ IR regions. The wavelength regimes for these region range from $100 \mathrm{~nm}$ to $400 \mathrm{~nm}$ for $\mathrm{UV}$, from $400 \mathrm{~nm}$ to $700 \mathrm{~nm}$ for visible, and then $700 \mathrm{~nm}$ to $100 \mu \mathrm{m}$ for IR region. Optical biosensors can also measure different optical properties such as energy, fluorescence, polarization, amplitude, decay time, and phase $[20,21,22,23,24,25]$. They can be used for in-vivo applications, since they are nonelectrical. They have the capability of allowing multiple analytes to be detected by using different monitoring wavelengths. Normally changes in wave propagation, time, wavelength, intensity, distribution of the spectrum, or polarity of the light are variables for detection. Devices based on fluorescence spectroscopy, interferometry, surface plasmon resonance are the most common $[19,13]$.

Generally Optical biosensing is categorized into two types: labelled detection and label free detection. Normally changes in fluorescence, and luminescence are used as physical variables for detection in labelled optical sensor technologies and refractive index changes for label free detection.

\subsubsection{Label free biosensors}

In label-free detection protocol, the biomolecules are not labeled or modified, they are detected in their natural forms. In this methodology the changes in peak with change in refractive index are used as sensing transduction. In label free detection, with the presence of the biological matter, it displaced the background fluid and changes the refractive index of the surrounding environment. This change in refractive index leads to the change in optical properties of the sensor. By observing the shift in spectral properties like change in reflectance, transmission, the presence of the biomolecule can be identified. There are various label free optical biosensing platforms like using photonic crystals [26], interferometers [27], surface plasmon resonance [28], waveguides [29] were developed. This has various advantages as it is very cheap and easy to 
perform, this also allows kinetic and quantitative analysis of molecular interaction. Label free detection mechanisms usually measure refractive index changes induced by molecular interaction [12].

\subsubsection{Label based biosensors}

In label based optical detection/ fluorescence based detection, bio recognition molecules/ target molecules are labeled with fluorescent dyes/ tags where intensity of fluorescence indicates the presence of target molecules. Labeled detection scheme is very sensitive type of detection which can detect even a single molecule [30]. But, it has a disadvantage of complex labeling process and attachment of label can change the behavior of the molecule to be detected [31]. Traditionally for DNA assays are based on measuring the emission of labeled target DNA, with the level of fluorescence emission (in relative fluorescence units (RFU)) proportional to the amount of material present.

\subsubsection{Fluorescence - based biosensors}

Fluorescence represents a widely used optical detection technique in current medical diagnostic technologies due to its sensitivity and selectivity. In fluorescence, a molecule is excited and detected to produce a bright signal that can be measured easily even at the single cell level. A molecule that is capable to fluoresce is called a fluorophore.

For DNA labeling, fluorophores that emit light in visible range $(400 \mathrm{~nm}-600 \mathrm{~nm})$ are used. After photons form a laser or other type of light source connected to am emission filter excites the molecule, the molecule descends back to ground state. Photon emitted from the laser has less energy in comparison with the input photon energy. Therefore, the wavelength of a photon emitted from a fluorophore has less energy than the wavelength of the photon delivered. Optical filters fitted onto the microscope so that the emitted light passes through and measured so that all the excitation light is filtered out. Generally, fluorescence detection is used to detect a specific molecule before and after reaction takes place. For medical applications, dye is attached to the target molecule which is to be detected, the dye will optically transduce to indicate the presence 
of the target molecule. Normally fluorescent tag, a fluorescent protein (FP) enables researchers to study the location, structure and dynamics of the molecular events in living cells [32].

\subsection{Photonic Crystals}

Photonic crystals are periodically varying dielectric nanostructures. This periodically varying dielectric potential of photonic crystals allows the existence of forbidden gaps which are called as photonic bandgaps. Varying the photonic crystal parameters; refractive index of the material, lattice constant, and periodicity, the frequency range of the light to be allowed, confined and blocked can be controlled. Physically, the periodic arrangement of varying dielectric constants can be in one/ two/ three dimensions. The refractive index and arrangement is engineered that only certain wavelengths can pass through the lattice of this arrangement. Photonic crystals can are described and modeled by Maxwell's equations.

One-dimensional photonic crystals have a dielectric material varying periodically in one direction. These have a smooth surface and reflect a specific wavelength. These are typically fabricated by spin coating, layer by layer deposition and photolithography [33, 34].

2 dimensional photonic crystals have periodically varying dielectric constant in two directions [35]. The fabrication process of $2 \mathrm{D}$ photonic crystals is a top to down process and it is generally fabricated by electron beam lithography, etching and nanosphere lithography [36].

3 dimensional periodic structures have the dielectric constant varying periodically in 3 dimensions. They can provide light confinement in all the 3 directions. 3D photonic crystals are periodic $3 \mathrm{D}$ arrangement of nanospheres. Typically, the spheres material used are zinc oxide, silica, polystyrene beads, titanium oxide, and polymethyl methacrylate [37].

Photonic crystals are used for various physical and chemical applications [38] including physical, biological, and chemical sensing. Photonic crystals can be used to design biosensors for label based and label free detection. 


\subsubsection{Photonic Crystal Biosensors}

To improve the limit of detection, sensitivity and selectivity taking advantage of specific optical bandgap by photonic crystals is used for biosensing. There are two types of detection modalities in photonic crystals: Active detection and passive detection.

\subsubsection{Active photonic crystal biosensors}

Active detection of biomolecules by photonic crystal is based on the principle, reflected light from the photonic crystals can be altered by change in refractive index or the change in the angle of incident light. In active detection, refractive index changes as the targets replace the low dielectric region of the photonic crystal. This change in refractive index leads to red/ blue shift in wavelengths. The shift in wavelength depends on the type of analyte. The analyte is detected by the shift in the peak refractive index wavelength [39].

\subsubsection{Passive photonic crystal biosensors}

In passive detection using photonic crystals, the label attached to the biomolecule is detected by the PhC. The light emitted from the label is controlled and guided to direct the photons towards the detector leading to the enhancement of the light emitted from the label by creating excess number of photons per unit time [40]. The fluorescence molecules attached to the biomolecules are detected even at low concentrations [41]. Various 2D photonic crystals have been modeled and fabricated for passive detection [42] [43] [44] [45]. A 2D photonic crystal structure was engineered by introducing holes of low dielectric material in silicon substrate and it is used for labeled detection of DNA. The periodic photonic crystal is designed to coincide the resonant reflection peak with the excitation wavelength of a fluorophore. 


\subsection{Fluorescence Enhancement comparison}
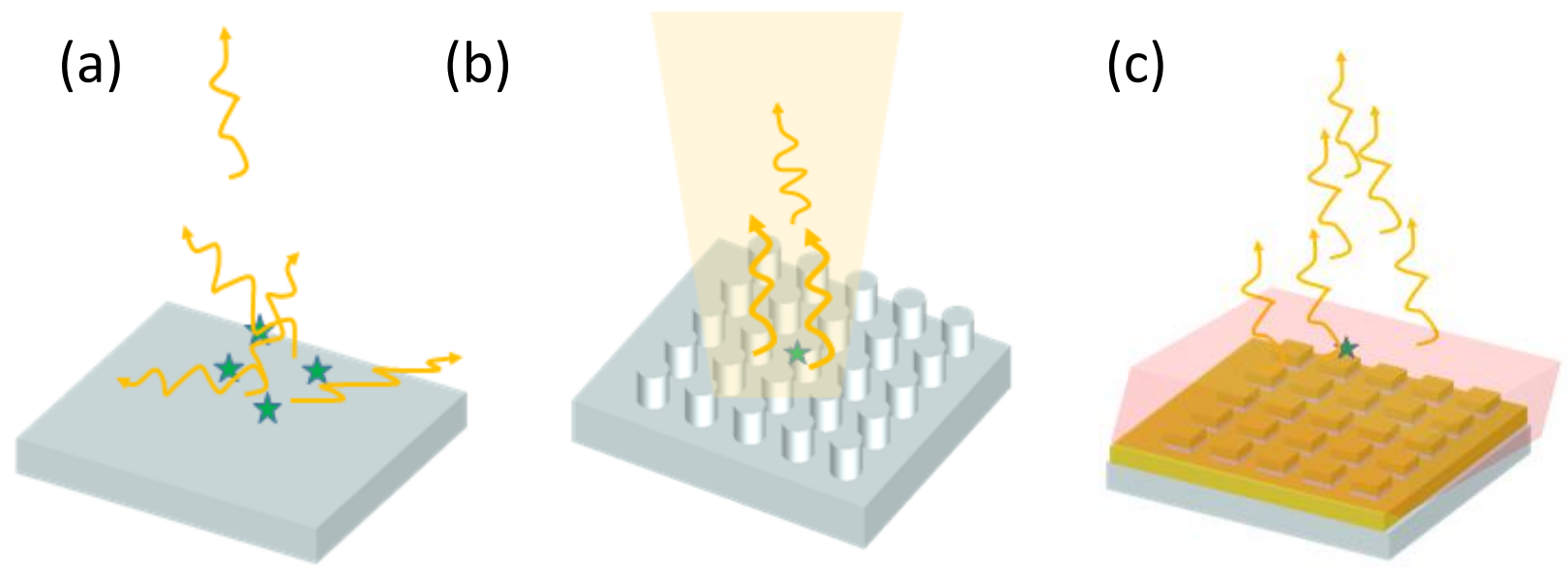

Figure 1-4: Fluorescence emission enhancement from the fluorophores on top of (a) a normal substrate (b) a photonic crystal (c) Plasmonic lattice structure

The Figure 1.4 shows the behavior of the fluorophores placed on the substrate. Figure (a) shows the fluorophores placed on a bare glass slide which emits photons in random directions, so number of photons reaching the detector are low. Figure (b) shows the fluorophores placed on the photonic crystal, the photons emitted from the fluorophores are directed towards the detector improving the number of photons detected per unit area. Figure (c) shows the fluorescent molecules placed on the top of a plasmonic lattice structure. Plasmonic lattice structure increases the electric field in its vicinity. The number of photons are emitted by the fluorescent molecule due to the electric field in the vicinity of the metal nanoparticle.

\subsection{Plasmonic biosensors}

The field of plasmonics is the study of the interaction between free electrons in a metal and incident light energy (i.e. photons). Plasmonics have applications in a variety of fields due to the advantage of light confinement. They are used in research areas like solar cells, PEC cells, etc [42]. For biosensing, plasmonic biosensors can be used for label based detection and label free detection schemes. The localized surface plasmon resonance peak is used as reference for label 
free biosensing, where the shift in the peak is notification for the presence of molecules. The presence of sensing molecules leads to the change in refractive index of the environment, which causes the shift in plasmonic resonance peak [43]. For label-based detection, biosensors taking advantage of the plasmonic properties of metal films and nanostructures have emerged as the alternative method for detection of DNA with lower cost and high sensitivity. There is a demand for compact, simple, low cost devices that can effectively detect very low concentration of biological molecules [44].

\subsubsection{Metal Enhanced Fluorescence}

One of the strategies to improve the detection sensitivity of fluorescence-based biosensors is by placing the fluorophore in the high electric field generated by confining the light with metal nanoparticles. This improves the detection sensitivity by enhancing the light emitted from the fluorophores. This whole process of enhancement in fluorescence due to metal nanostructures is called as metal enhanced fluorescence (MEF) [8].

Metal enhanced fluorescence is a special case of plasmonic biosensors, which involves the study of fluorophores in the vicinity of metal nanostructures. The increased electric field around the nano-patterned metal improves the enhancement from the fluorophores, which in turn improves the sensitivity of detection. LSPR sensors employ noble metal nanostructures for sensing applications they are defined as plasmonic nanosensors. The localized surface plasmon resonance in gold nanoparticles can be used as the basis for high-sensitivity label-based detection. The fluorescence enhancement is due to the localized surface plasmon polaritons associated with metal nanostructures, which increase the electric field in the vicinity of the nanostructure. The fluorescent molecules are modified by the increased electric field around the nanostructures. The increased electric field creates an additional radiative decay source when the fluorescent molecule is in the vicinity of metal nanostructures. However, there are various other factors on which the enhancement factor depends on like, quantum yield of the dye, concentration in solution [9]. Additionally, metal nanostructures are known for their ability to interact with organic compounds to improve significantly the photo stability and fluorescence intensity of many fluorescent dye molecules [45]. Hence metal enhanced fluorescence is widely 
investigated for labeled biosensor applications particularly for fluorescent dyes with low quantum yield.

\subsubsection{Applications of Metal Enhanced Fluorescence}

\subsubsection{MEF in labeling proteins}

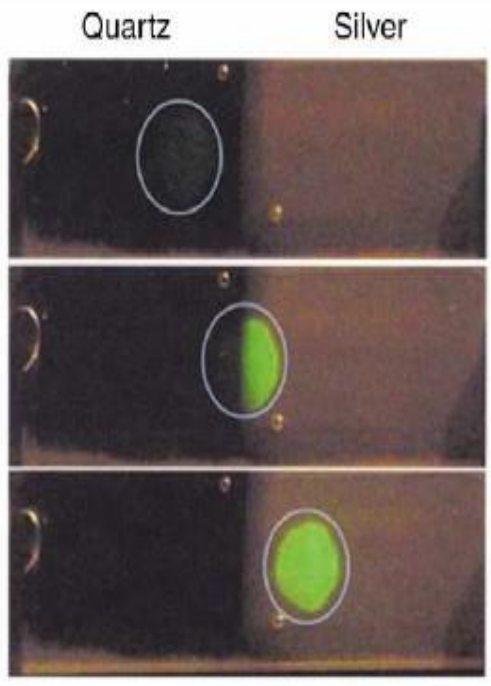

Figure 1-5: Photograph of fluorescein-labeled HSA on quartz (left) and on SIFs (right) as observed with laser excitation of $430 \mathrm{~nm}$ excitation long pass emission filer at $480 \mathrm{~nm}$. The excitation was progressively moved from the quartz side to the

Fluorophores have been prominently used as probes in biological research to identify protein location and detect protein complex formation for in vivo monitoring of biological processes. The most commonly used fluorophore for this application is fluorescein dye. But this dye is often subjected to quenching due to foster resonance energy transfer between neighboring fluorescent molecules [46]. This problem limits the labeling density, which in turn decreases the intensity of the labeling protein. It is proved that self-quenching is eliminated by placing the dye in proximity to the silver island films. This reduction in self-quenching is due to the increase in radiative 
decay rate of the fluorophores in the vicinity of the metal. The experimental investigations were performed with fluoroescein-5-isocyanate-labeled human serum albumin (FITC-HSA). As part of experiment, FITC-HSA was examined when it is bound to glass (quartz) and to Silver Island Films (SIF's). It is proven that the emission intensity is about 17 times higher in the presence of SIFs than the glass alone as shown in the Figure 1.1 [46]. The increase in radiative decay and decrease in self-quenching is due to metal enhanced fluorescence caused by colloidal silver. Hence this proves that metal enhanced fluorescence can produce ultra-bright fluorescent probes for use in biological detection [46].

\subsubsection{Metal Enhanced Fluorescence for Medical Imaging}
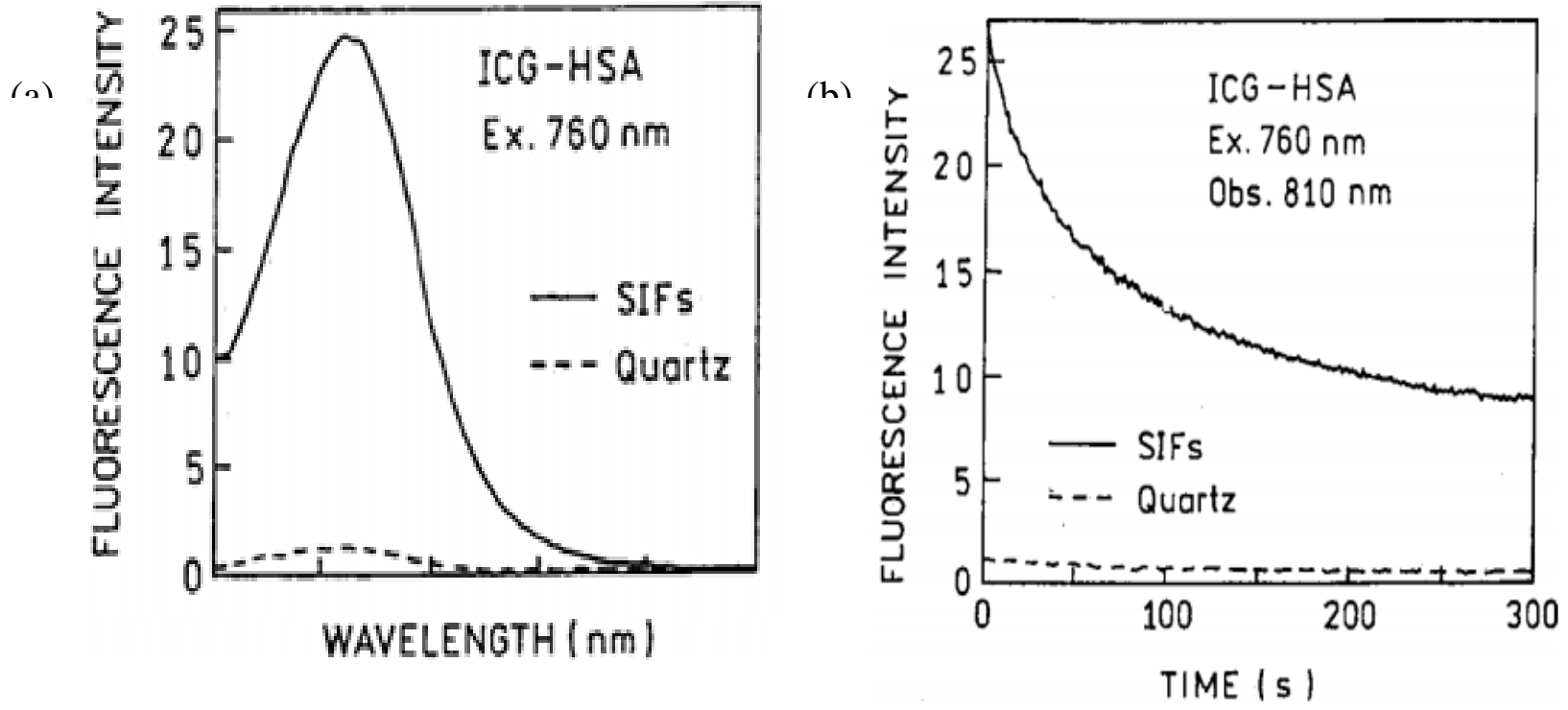

Figure 1-6: (a) Emission spectra of indocyanine green-albumin (ICG-HSA) bound to unsilvered quartz slides or silver island films. (b) Photo stability of ICG-HSA on quartz and on SIFs

Indocyanine green (ICG) is a non-toxic dye approved by Food and Drug Administration (FDA) for medical imaging applications like optical detection of tumors [47], retinal angiography [48], optical tomography [49]. The dye absorbs and emits the light in the near infrared spectrum. The ICG dye bound to human serum was tested on silvered and unsilvered surfaces [44]. The silver islands were deposited by chemical reduction of silver nitrate on the glass substrate. The 
diameter ranged of the silver islands ranged from $100 \mathrm{~nm}$ to $500 \mathrm{~nm}$ and about $60 \mathrm{~nm}$ in height. The emission intensity of ICG dye in the proximity of silver island films (SIFs) is 20 times higher when compared to the bare quartz surface as shown in Figure 1.2(a). The fluorescence enhancement is due to the higher rate of excitation near the silver particles. Moreover, the increase of photo stability is observed, due to the reduction in photo bleaching is observed with time near the silver particles as shown in the Figure 1.2(b). Metal colloids have good scattering and they can enhance the fluorescence, so they have dual effect that could dramatically improve the detection in tissues [44].

\subsubsection{Metal Enhanced Fluorescence in DNA hybridization}

In biotech and diagnostic applications, detection of DNA hybridization is important for polymerase chain reactions (PCR). The general approach is to change the fluorescence intensity upon hybridization. Thiolated oligonucleotide SS DNA bound to silver nanoparticles on glass substrate were investigated and are compared with SS DNA in solution. A complementary labeled fluorescein oligonucleotide was added to solution containing silver-bound DNA.

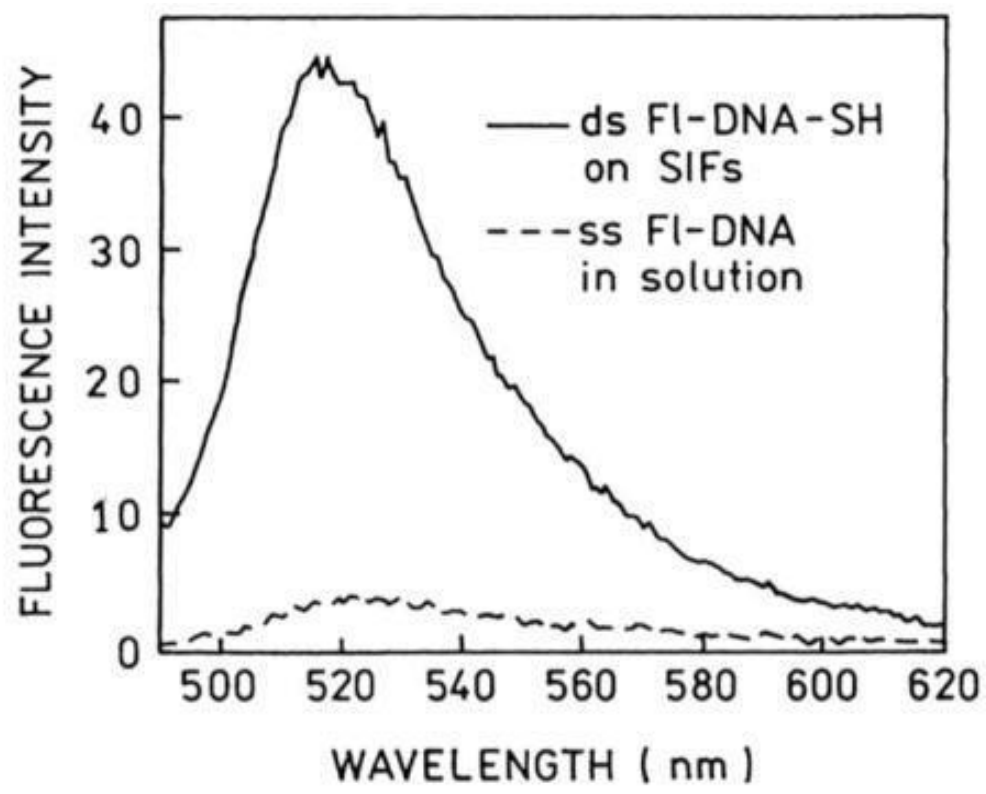

Figure 1-7: Emission spectra of SS DNA bound (solid line) to silver nanoparticles and in solution (dashed line). 
The proximity of F1 DNA to silver nanoparticles during hybridization with the capture resulted in 12-fold increase in fluorescence intensity as shown in the Figure 1.3 and decrease in fluorescence life-time [50]. The increase fluorescence intensity is observed, hence improving the sensitivity of detection [50]

\subsubsection{Metal enhanced fluorescence for luminescence enhancement quantum dots:}

Metal enhanced fluorescence can be used to improve the luminescence of the colloidal nanocrystals with the nanoscale gold triangular nanopillars [51]. A square array of triangular gold nanopillars of height $40 \mathrm{~nm}$ and lateral dimension of $200 \mathrm{~nm}$ and period of $400 \mathrm{~nm}$ is

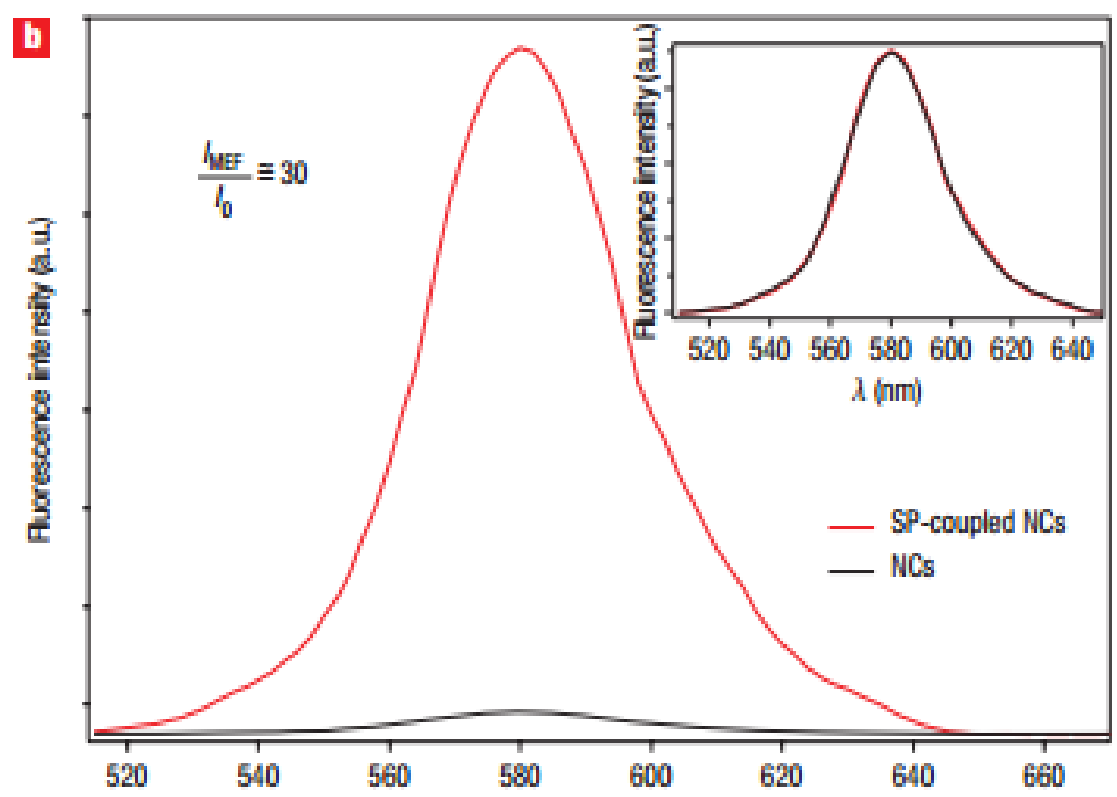

Figure 1-8:Enhancement factor of 30fold on the gold nanopillars compare to the background (silicon substrate)

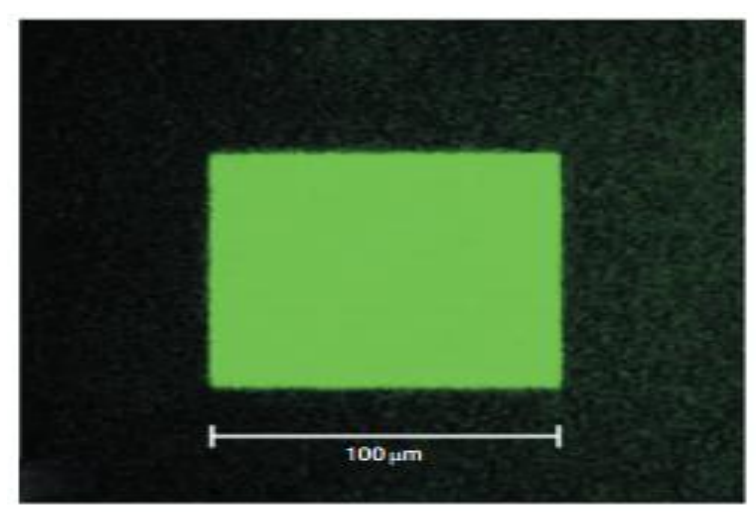

Figure 1-9: Enhancement of the quantum dots on the gold nanopillars compared with the silicon substrate 
fabricated. A strong enhancement in luminescence of the quantum dots mixed in polymer PMMA spun over the gold nanopillars is observed. An enhancement factor of 30fold is observed onthe gold nanopillars compared to the silicon substrate is observed.

\subsubsection{Metal enhanced fluorescence for reduction in photobleaching}
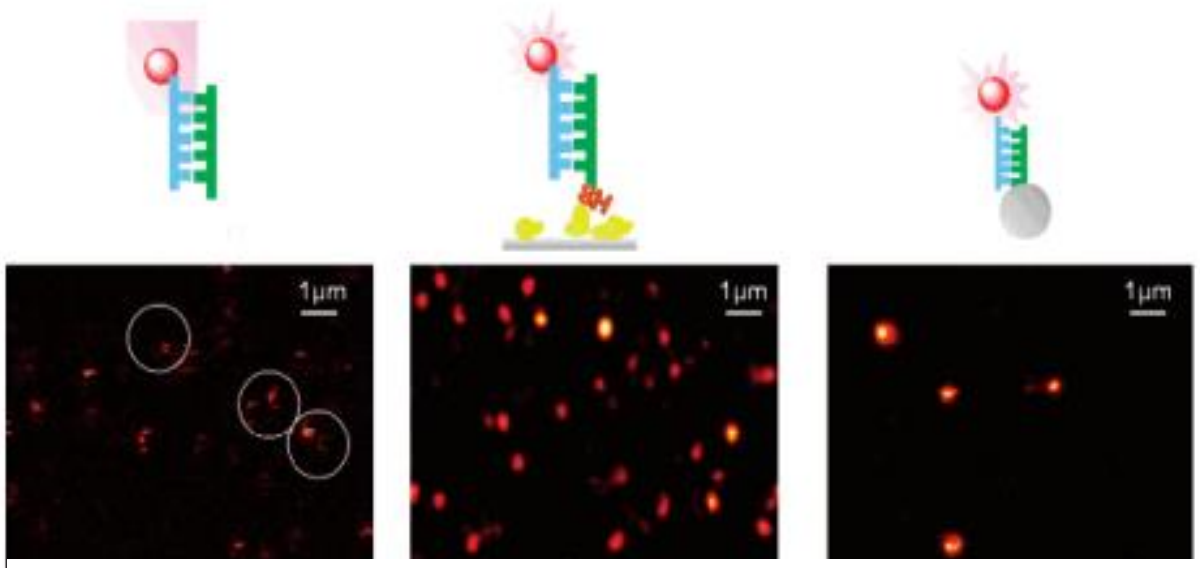

Figure 1-10: (a) Cy5 dye labeled DNA spin coaeted on glass (b) Cy5 dye labeled DNA tethered to SIFs (c) Cy5 dye labeled DNA coupled to silver nanoparticles

Metal enhanced fluorescence showed applications in reduction of photobleaching and random blinking in fluorophores. The organic fluorophore Cy5 dye has random blinking behavior and irreversible photobleaching. Cy5 dye placed in close proximity to the silver islands and when coupled to the slver nanopartciles showed reduction in photobleaching. The metal- fluorophore interaction greatly suppressed the blinking of $\mathrm{Cy} 5$ dye and also reduced the photobleaching and enhanced the photostability. The surface plasmon resonances from the silver nanoparticles modified the spectral properties of Cy5 dye.

\subsection{Scope of this thesis}

The work presented herein, a new SEF (surface enhanced fluorescence) substrate fabricated by ebeam lithography is demonstrated which utilizes a 2 dimensional array of gold square 
nanopillars. An average fluorescence enhancement of 2.01 fold is achieved on the plasmonic nanostructures compared to the flat gold surface.

Simple DNA detection equipment requires a microscope to visualize DNA under a microscope. DNA applications require DNA labeled with dyes emitting in visible spectrum. Plasmonic enhancement is directly proportional to the wavelength of the emission.

The array is engineered to achieve a high enhancement factor due to plasmonic resonance, which is influenced by the physical parameters of the plasmonic lattice. Tailoring of the resonant optical properties of plasmonic nanostructures requires an understanding of the physical parameters that influence the plasmonic resonances. A finite difference time domain (FDTD) software tool developed by Lumerical was used to understand the dependence of electric field enhancement on the peak resonant wavelength, spacing between the nanostructures, and thickness of gold deposition. Simulations of the plasmonic crystal designed to provide the enhancement peak at 560nm are described in Chapter 3. The critical dimensions of the simulated device were extracted and used in fabrication processes, which are presented in Chapter 4. Electron-beam lithography was used to realize 2D plasmonic square nanopillars on a semiconductor (silicon) substrate.

The plasmonic lattice was created using gold deposition. The fabricated nanostructures were inspected and analyzed by using high-resolution scanning electron microscope to achieve desired geometrical parameters, required by the design.

Chapter 5, presents the results of fluorescence enhancement characterization. The fabricated substrates were used to enhance the fluorescence of the $\mathrm{CdSe} / \mathrm{ZnS}$ quantum dots with an excitation wavelength of $565 \mathrm{~nm}$. The fluorescence enhancement of the fabricated samples are tested by drop coating the quantum dot solution on the top of fabricated plasmonic nanostructure using a confocal microscope with laser excitation of $543 \mathrm{~nm}$

Finally, overall conclusions of the thesis and future work are discussed in chapter 6. 


\section{Chapter 2}

\section{THEORITICAL REVIEW}

This chapter explains the theory upon which this thesis project is based, specifically, the exploitation of light interaction with metallic nanostructures and the effect of metal nanostructures on fluorescent dye molecules.

For centuries, metal colloids were used to make colored glass [52]. In fact, colloidal gold was responsible for origin of color in medieval cathedral windows. The experimental investigation of interaction of light with metal nanostructures was first recognized by Faraday in 1857 using a beaten gold leaf with $\frac{1}{282000}$ th of an inch in thickness, where the gold leaf is translucent, reflecting yellow, transmitting green light and absorbing a portion [53]. Localized surface plasmons (LSPs) are collective charge density oscillations which are excited when a light at resonance wavelength is incident on the metal nanoparticle [54]. These are confined to surface of metallic nanostructures. This phenomenon causes strong light scattering and local electromagnetic field enhancement. The localized surface plasmon resonance wavelength and surface plasmon extinction peak intensity depend on the type of material, size and shape of the nanostructures $[55,56]$. These properties of metallic nanostructures have been used for creating optically sensitive biosensors, and as molecular recognition elements, sensors, solar cells, and photo electro chemical (PEC) cells [42]. This thesis emphasizes on the interaction of metallic nanostructures with fluorophores to create unique optical labelled biosensors [57].

Metal enhanced fluorescence (MEF) or plasmon enhanced fluorescence (PEF) describes the interaction of light with metallic nanoparticles which in turn effect the fluorescent dyes. This 
theory can be categorized into two parts: the resulting effect of the light incident on metallic nanostructures and interaction of the nanostructures with fluorescent dye molecules [13].

The phenomenon of increase in electric field in the proximity of metal plays an important role in the enhancement of fluorescence of a dye molecule. The increase in electric field depends on the shape of the nanoparticle. Normally this increase is about 2 orders of magnitude of electric field higher at the proximity of the nanoparticle compared to magnitude of electric field of incident photons. But the magnitude of electric field decays as the distance from the metal nanostructure increases.

\subsection{Plasmonics:}

The field of plasmonics is the study of interaction between the free electrons in the metal and electromagnetic field produced by light.

Light is an electromagnetic wave which has transverse electric field and magnetic fields. On applying an incident light to the metal nanoparticle, it will displace the free electrons from their corresponding nuclei - their equilibrium position. This displacements of charges lead to the formation of an electronic and an ionic cluster on the surface of the particle: a dipole, induced by the incident light. The charges will follow the harmonically oscillating external field. Therefore the dipole will oscillate and hence will radiate electromagnetic waves. Localized surface plasmon resonance occurs when the oscillating plasmon resonance equals the wavelength of the incident electromagnetic wave [58]. The radiated electromagnetic waves will increase the electric field in the vicinity of the metal nanostructures.

\subsubsection{Surface Plasmons}

Solid state physics defines surface plasmons are the electromagnetic oscillations that propagate along the metal-dielectric interface. As part of electrodynamic theory, surface plasmons are defined as a particular case of surface wave. As per as optics, surface plasmons are modes of an interface [59]. When noble metal nanoparticles are incident with electromagnetic radiation, the surface electron cloud on the top of the metal oscillates coherently relative to the incident electromagnetic radiation, which causes the displacement of charge cloud from the nanoparticle. 
This coherent motion of electrons on the surface causes a wave. Hence this wave is called a surface plasmon wave.

The electromagnetic waves oscillate with the frequency of the incident light. A dipole approximation is used to describe the optical properties of sub wavelength metal nanoparticles. Mie theory is used to describe the optical properties of spherical metal nanoparticles.

\subsubsection{Localized Surface Plasmon Resonance (LSPR)}

Localization of the plasmon resonances between the nanostructures is by patterning which results in the higher surface area causing scattering to be higher in a patterned structure. LSPR depends on size, shape, material, refractive index of the environment. Change in size and shape and material can result in tunable plasmonic peak which can be controlled. The electric field around the nanoparticle is highly charged compare to the incident electric field. The light scattering by the metal nanoparticle increases the optical path length which, enhances the probability of light absorption.

\subsection{Plasmonic Theory}

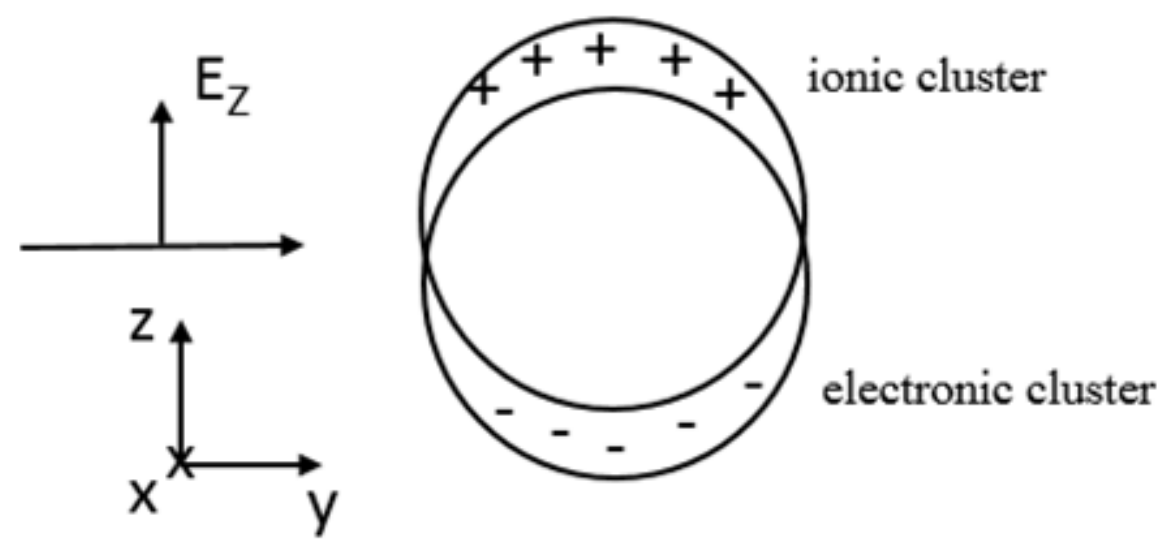

Figure 2-1: Induced dipole moment in a metal particle with an external electromagnetic field 
The plasmonic phenomenon occurs only under specific conditions:

a) The material permittivity of the metal $\varepsilon_{m}$ is a complex function $\left(\varepsilon_{m}=\varepsilon_{r e}+i \varepsilon_{i m}\right)$ of frequency, surface plasmons occurs only when the real part is negative in the frequency range.

b) The exciting freespace wavelength is large compared to the particle dimensions

The extinction energy which is the sum of scattering and absorption energy can be evaluated by mie theory for spherical nanoparticles. The theory of plasmonics can be split up by means of two different aspects: electromagnetic theory and theory of material properties of metals. The complex dielectric constant is the connection between these two parts.

\subsection{Mie theory}

The metallic sphere when irradiated by an incident electromagnetic field generates electric field around it which was solved and published in 1908 by Gustav Mie [60]. Mie theory is the rigorous mathematical solution for 'the scattering by a dielectric sphere' problem by means of solving Maxwell's equations. Scattering, excitation of surface plasmon polaritons, and penetration into the material, etc are completely covered by Maxwell's equations. Mie theory describes a small metal nanoparticle can be approximated by a radiating dipole, as shown in Figure 2.1. Free electron gas model is used to describe the behavior of electrons in a metal. The interaction of spherical nanoparticles and electromagnetic waves is explained by Mie's theory [10].

\subsubsection{The Radiating Dipole}

A result of the Mie theory is, that a small particle can be approximated by a radiating dipole. In Figure 2.1 this is illustrated. An external electric field will displace the free electrons from their corresponding nuclei - their equilibrium position. This displacements of charges lead to the formation of an electronic and an ionic cluster on the surface of the dipole. The charges will follow the harmonically oscillating external field. Therefore the dipole will oscillate and hence will radiate electromagnetic waves. The charges will follow the harmonically oscillating external field. Therefore the dipole will oscillate and hence will radiate electromagnetic waves. For small 
particles, i.e. $l<<\lambda$, the incident electric field penetrates considerably through the particle. The particle is placed in a homogeneous electric field E-. The particle gets polarized and generates a field, which modifies the total field. The induced dipole moment is described by

$$
\vec{p}=\alpha \vec{E}
$$

Equation

\section{1}

where, $\vec{p}$ Induced dipole moment, $\alpha$ is polarizability, $\vec{E}$ is Electric field strength of the incident electromagnetic wave

Actually, this relation defines the polarizability of a particle. The polarizability of a sphere can be obtained by solving the Laplace equation and is given by the Clausius-Mosotti relation

$$
\alpha_{\text {sphere }}=4 \pi a^{3} \varepsilon_{o} \frac{\varepsilon_{1}-\varepsilon_{m}}{\varepsilon_{1}+2 \varepsilon_{m}}
$$

Equation

where a is the radius of the nanoparticle, $\varepsilon_{i}$ complex electric permittivity of the metal, $\varepsilon_{S}$ complex electric permitivity of the surrounding medium, $\varepsilon_{0}$ Electric permittivity of the vacuum.

The absorption cross-section of a spherical metal nanoparticle placed in a transparent dielectric matrix, where the imaginary part of the relative complex electric permittivity approaches zero $\left(\operatorname{Im}\left[\varepsilon_{s} \perp->0\right)\right.$, is then given as:

$$
\sigma(\omega)=12 \pi R^{3} \frac{\omega}{C} \varepsilon_{s}^{\frac{3}{2}} \frac{\varepsilon_{i}^{i i}(\omega)}{\left[\varepsilon_{i}^{i}(\omega)+\varepsilon_{s}\right]^{2}+\varepsilon_{i}^{i i}(\omega)^{2}}
$$

$\varepsilon_{i}{ }^{i}(\omega)$ real part of electric permittivity of the metal, $\varepsilon_{i}{ }^{i i}(\omega)$ imaginary part of electric permittivity of the metal, $\varepsilon_{S}$ complex electric permitivity of the surrounding medium. 
The complex electric permittivity can be described by Drude-Sommerfeld formula in the Equation 2.4

$$
\varepsilon_{i}(\omega)=\varepsilon_{b}+1-\frac{\omega_{p}}{\omega^{2}+i \gamma \omega}
$$

$\gamma$ is the damping constant of electron oscillation, $\varepsilon_{b}$ complex electric permittivity associated with interband transitions of the core electrons of the atom, $\omega_{p}$ is the free electron plasma frequency. Equation 2.5 gives equation of plasma frequency

$$
\omega_{p}=\sqrt{\frac{N e^{2}}{m \varepsilon_{0}}}
$$

Where $\mathrm{N}$ is the density of free electrons, $\mathrm{M}$ is the effective mass of the electron

Mie resonances occur at surface plasmon frequency $\omega_{s p}$ only under the following conditions

$$
\left[\varepsilon_{i}^{i}(\omega)+2 \varepsilon_{s}\right]^{2}+\varepsilon_{i}^{i i}(\omega)^{2} \rightarrow \text { Minimum }
$$

Equation

If the condition in the Equation 2.6 is fulfilled, then the dipole moment is produced and the local electric field in the vicinity of the nanoparticle grows. The magnitude of the electric field enhancement can be many orders more than the incident wave.

$$
\varepsilon_{i}^{i}\left(\omega_{s p}\right)=-2 \varepsilon_{s}
$$

The above condition requires the equation to be minimum, if the imaginary part of metal electric permittivity is small in comparison with the real part. Equation 2.7 requires the real part of dielectric function of metals to be negative. 


\subsection{Extinction power}

Extinction is the sum of absorption and scattering power. If the scattering power dominates, then the metal enhanced fluorescence occurs. If absorption dominates, it results in quenching of fluorescence. According to literature, for nanoparticles greater than $40 \mathrm{~nm}$, scattering dominates. And for nanoparticles less than 40nm, absorption dominates.

\subsection{Fluorescence Spectroscopy}

Fluorescent spectroscopy is widely used in applications related to chemistry, photonics and material science, and molecular biology. Fluorescence is defined as the process of absorption of energy of light at one wavelength and nearly instantaneous re-emission at a longer wavelength. Surface enhanced fluorescence consists of modification of fluorescence intensities and lifetimes of the molecules on the metal surface [61] [62]. This is due to the metal particles resulting in strong electromagnetic (EM) response.

Fluorescence biosensors work based on the emitted wave produced by the dyes attached to the analytes or biomolecules. The emission intensity of the dye is the limitation for the detection in labeled biosensor detection. Metal nanoparticles can be used to improve the fluorescence intensity of the dye in their close proximity. When a fluorophore is brought in close proximity to the metallic nanostructure, the intensity of the fluorescence of the fluorophore has been shown to increase dramatically and the fluorescence lifetime of the fluorophore has been shown to decrease, resulting in an increased photostability. This phenomenon is known as Metal Enhanced Fluorescence (MEF). This phenomenon was first invented and named by Geddes and Lakowicz in 2002 [63]. MEF is caused by the non-radiative coupling of the fluorophore dipole with the electron cloud of the metal (surface plasmons), there by altering the characteristics of the fluorophore. MEF is a near-field phenomenon which occurs only when a fluorophore is within $10-100 \mathrm{~nm}$ of a metal which supports surface plasmons [45].

Fluorescence involves two processes, adsorption of photon, followed by emission. This process is similar to scattering. The enhanced local electric field leads to the change in absorption cross section, but the modification in the decay rates can be only modified by radiative enhancement. 
Surface Enhanced Fluorescence is the enhancement of the fluorescence molecules in the vicinity of the nanoparticles. The presence of the nanoparticles reduces the lifetime of the molecule in the excited state. The theoretical explanation for fluorescence enhancement and the experimental results defines that the enhancement can occur only for fluorophore - metal enhancement can occur for distances greater than $10 \mathrm{~nm}$. The spacer layer leads to increase in relaxation rate and it helps to undergo more excitation and emission cycles before photo bleaching.

\subsubsection{Photophysics of fluorophore}

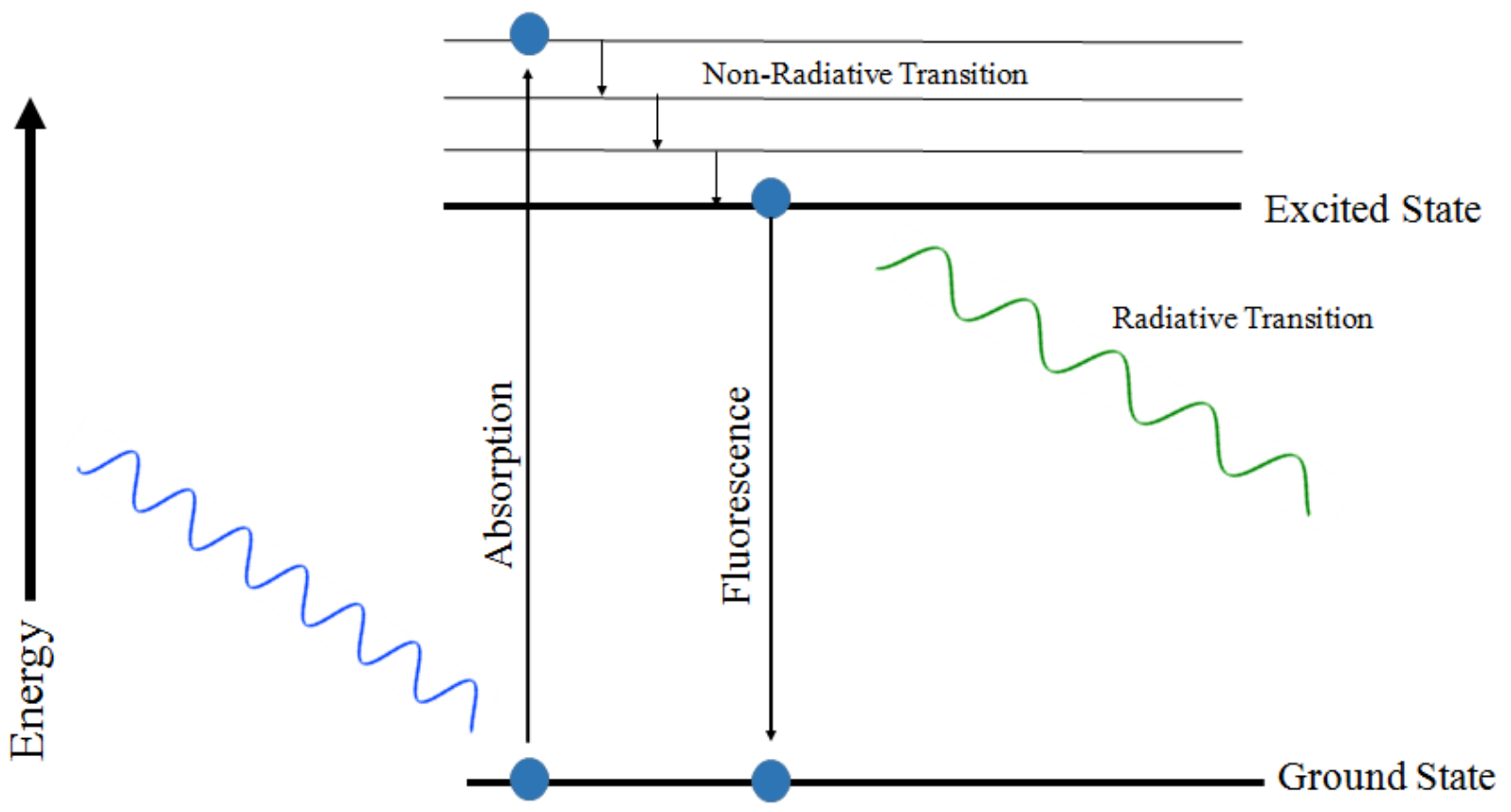

Figure 2-2: Process of fluorescence

Fluorescence is a property of some molecules or atoms to absorb light at a particular wavelength and emit the light at a longer wavelength than the incident light. When the fluorescence molecule absorbs light, the electron is excited form ground state to the higher energy state. In the higher energy state, the electron loses some of the energy in the form of vibrational energy which is 
non-radiative transition, vibrational relaxation is the emission of infrared radiation (lower energy) the high energy electron returns to the ground state by emission of photon which is a radiative transition. The shift in the wavelength between the absorption and the emission spectra is called as the strokes shift. The extinction coefficient of the fluorophore is the intrinsic ability of the fluorophore to absorb the light at the given wavelength. Quantum yield of the fluorophore is the ratio of the radiative decay rate to the total decay rate (radiative and non-radiative decay) rate of the fluorophore as in Equation 2.8.

$$
Q=\frac{k_{r}}{k_{r}+k_{n r}}
$$

Equation 2.8

where $\mathrm{Q}$ is quantum yield, $k_{n r}$ is non radiative decay rate, $k_{r}$ is radiative decay rate

Lifetime of the fluorophore is the average value of time spent in the excited state as in Equation 2.9.

$$
\tau=\frac{1}{k_{r}+k_{n r}}
$$

where $\tau$ is Lifetime of the fluorophore, $k_{n r}$ is non radiative decay rate, $k_{r}$ is radiative decay rate

Laser excited fluorescence is widely used for biological applications as the fluorescence signal can be significantly red-shifted (strokes shift) from the excitation wavelength, resulting in rendering a background free signal. The intensity of fluorescence depends on the spontaneous emission of photons, isotropically in all directions. As the emission of photons is isotropic, the efficiency does not depend on controlling the observation direction and the collection optics. The information of the sample is obtained by the radiative decay rate $k_{r}$ and non-radiative decay rate $\left(k_{n r}\right)$. The changes in $k_{r}$ and $k_{n r}$ result in change of life time and intensity. Introducing a fluorophore in the vicinity of metal adds an additional radiative decay rate, $k_{m}$. The fluorescence rate from a fluorophore $\Gamma_{f l}$ is determined by the intrinsic quantum yield $Q_{i}$ and the excitation rate $\Gamma_{\text {exc }}[64]$. 


\subsection{Enhancement of Fluorescence}

\subsubsection{Plasmon- Dye Interactions}

Metal nanoparticles increase the fluorescence intensity of the fluorophores located in their proximity. To explain this phenomenon, two mechanisms have been proposed. The first mechanism, Energy transfer between fluorophore and the metal nanoparticle, resulting in surface

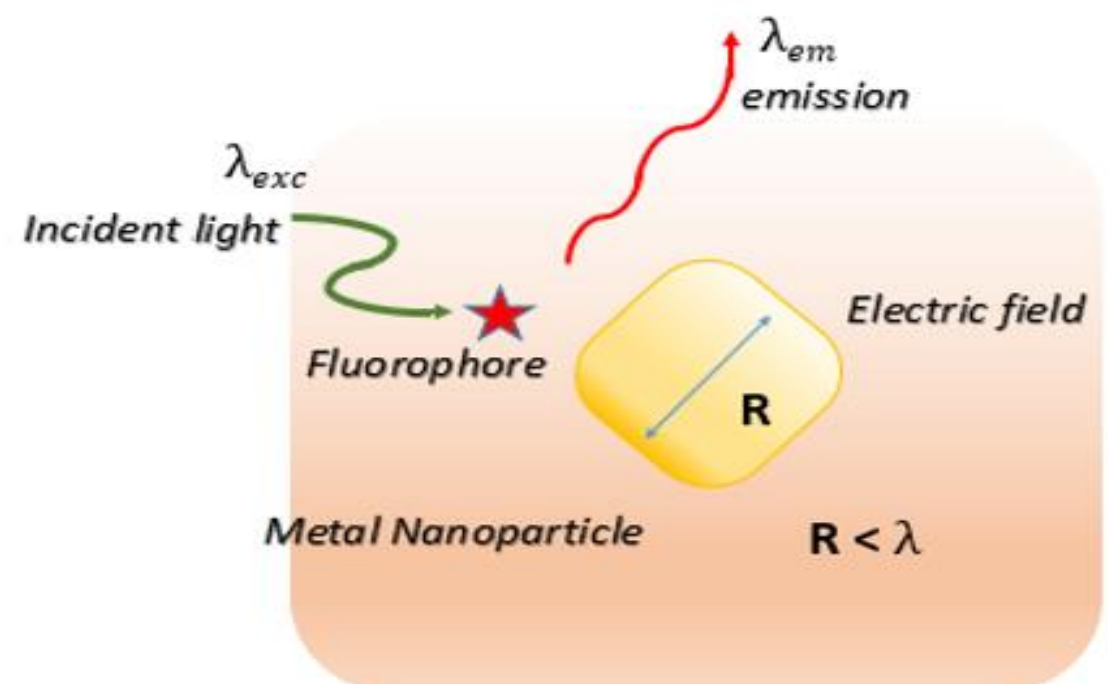

Figure 2-3: Interaction of fluorophore with the electric field generated by gold nanoparticle

enhanced fluorescence. The effective energy transfer occurs if the absorption wavelength of the fluorophore matches with the surface plasmon resonance wavelength of the metal nanostructure. In order to obtain an effective transfer, the surface plasmon resonance peak of the nanoparticles can be tuned by monitoring the physical dimensions and the material composition of the particles.

The second mechanism, the enhancement of the fluorophore molecules results in the decrease of the radiative and non-radiative decay lifetimes due to coupling of the dye dipole moment with the electric field induced by surface plasmon resonance [64]. The light coupled as surface plasmon in a metal nanostructure, induces an electric field around the nanoparticle which decays 
with the distance away from the nanoparticle. The distance between the fluorophore and the nanoparticle is crucial in both the cases.

\subsubsection{Energy Transfer}

When a fluorophore is located in the vicinity of the metal nanostructure, the energy levels of the fluorophore and the surface plasmons may couple, resulting in the energy transfer between them as shown in Figure 2.3. The energy transfer may be observed as the increase in the radiative decay rate of the fluorophore with an additional radiative decay channel added due to the metal nanostructure.

There are two different scenarios occurring between dyes and nanoparticles with the interaction of the light (shown in the figures)

a) When the excited electron in the dye molecule relaxes to the ground state, it releases some energy. The energy from the dye molecule is received by the metal nanoparticle which acts as the reservoir. So, rather than the far field radiation of the energy emitted, most of the energy is transferred to the metal nanoparticle which results in quenching. This energy is transferred to metal nanoparticle to excite surface plasmons. Hence very few dye molecules generate far field radiation by returning to the ground state by radiative decay path. But this decreases the lifetime of the molecules in the excited state and the molecules rapidly drop to the ground state. So this increases the population of electrons in the ground state. So more electrons are available for excitation. Hence the dye molecules in ground state absorb additional photons is increased, which results in the enhancement of the absorption of the dye.

b) The dye molecule is normally excited by the incident light, but in metal enhanced fluorescence there is an additional energy transfer from the optically excited localized surface plasmon polaritons. Metal nanoparticle has higher absorption cross section compared to the dye molecule, hence when the metal transfers energy to the dye molecule, an enhancement of fluorescence is expected. For the transfer of energy from metal to fluorophore, tow resonance conditions exist:

1. Energy level of surface plasmon is equal to upper energy level of the dye molecule 
2. Energy level of surface plasmon is equal to radiative transition of the dye molecule

In the first scenario, the transfer of energy can either happen from excited surface plasmons to the ground state of the dye molecule, leading to enhancement in fluorescence or the energy from the relaxed dye molecule in the excited state, excited the surface plasmons in the metal nanoparticle, which leads to surface enhanced absorption or quenching. Enhancement or quenching depends on the excited molecule and the life time of surface plasmon polaritons. The lifetime of the fluorophore decreases in the presence of metal nanoparticles. The reduction in lifetime depends on the material composition of the nanostructure and its dimensions and the separation between the nanoparticle and dye molecule [65]. Lifetime of the surface plasmon polaritons in gold and silver nanostructures is between 25fs and 800fs depending upon the structure [66].

The second scenario, leads to the quenching of the fluorescence as the energy transfer from the excited dye molecules in the intermediate energy level to the energy level of the surface plasmon occurs rather than relaxing to the ground state of the fluorophore. This energy transfer from the dye molecule to the metal nanoparticle decreases as the separation of the spatial distance increases. Quenching decreases with cube of the distance between the dye molecule and the metal surface. So the distance between metal nanoparticle and dye molecule needs to be between 10 and $30 \mathrm{~nm}$.

\subsubsection{Electric Field effects}

As discussed earlier, localized surface plasmons are produced by coupling of electromagnetic radiation with the metal nanoparticle. This surface plasmons which are localized to the surface of metal nanoparticle induces the electric field near the surface of nanoparticle and the electric field intensity decreases away from the surface of the metal nanoparticle. Maximum electric field enhancement is achieved at the localized surface plasmon resonance frequency. The manipulation of radiative decay rate of the dye molecule in the vicinity of the metal nanostructures is referred as Radiative decay engineering

The quantum yield $Q_{0}$ and lifetime $\tau_{0}$ of a fluorophore in the absence of the metal surface. 


$$
\begin{gathered}
Q_{0}=\frac{\Gamma}{\Gamma+k_{n r}} \\
\tau_{0}=\frac{1}{\Gamma+k_{n r}}
\end{gathered}
$$

$Q_{0}$ quantum yield of the fluorophore in the absence of metal surface, $\tau_{0}$ life time of the fluorophore in the absence of metal surface $\Gamma$ - Radiative decay rate of fluorophore, $k_{n r}$ - Non radiative decay rate of the fluorophore

The natural life time of the fluorophore is the inverse of radiative decay rate $\tau_{N}=\frac{1}{\Gamma}$. This is the life time that is observed when the non-radiative decay rate is equal to $0\left(k_{n r}=0\right)$. From the above equations, we can evaluate that the dye molecules with high radiative decay rate has high quantum yield and very short life times. The radiative decay rates are constant for a fluorophore, internally. But externally it depends upon the surrounding medium. Hence, for a fluorophore in a particular medium the radiative decay rate of it is considered to be constant. Hence quantum efficiency may be increased by reducing the non-radiative decay rate $\left(k_{n r}\right)$.

In the vicinity of the metal, due to the near field enhancement, the radiative decay rate is increased by the addition of one more radiative decay channel $\left(\Gamma_{m}\right)$. Hence quantum yield and the life time of the dye are modified by

$$
\begin{gathered}
Q_{m}=\frac{\Gamma+\Gamma_{m}}{\Gamma+\Gamma_{m}+k_{n r}} \\
\tau_{m}=\frac{1}{\Gamma+\Gamma_{m}+k_{n r}}
\end{gathered}
$$

$Q_{m}$ is the quantum yield of the fluorophore in the presence of the metal, $\tau_{m}$ is the lifetime of the fluorophore in the presence of the metal 
$\Gamma$ - Radiative decay rate of fluorophore, $k_{n r}$ - Non radiative decay rate of the fluorophore, $\Gamma_{m}-$ Radiative decay rate of fluorophore in the presence of the metal

The increase in the radiative decay rate of the fluorophore, the quantum yield increases and the life time decreases. As we can see the quantum yield fraction has highest value of one, if a fluorophore has highest quantum yield, the additional radiative decay rate induced by metal cannot result in much change of the quantum yield. If a dye has low quantum yield, then the additional radiative decay rate induced by the metal will increase the quantum yield enormously [67].

Microscopic theory of radiative transition explains gives further understanding of radiative decay rate . In this theory, a metal nanoparticle in the proximity of a fluorophore acts as a mirror and it causes the emitted photon to bounce back from the dye. This bouncing of photons increases the interaction of photons with dye molecules. This forces them to drop to ground state by simulated emission. The quantum mechanical transition probability $W_{i j}$ describes the probability that dye molecules will make a simulated jump to ground state. This is given by Fermi's Golden rule:

$$
W_{i j}=\frac{2 \pi}{h}\left|\mu_{i j}\right|^{2} \rho\left(v_{i j}\right)
$$

$\mu_{i j}$ - Transition dipole moment, $h$ - Planck's constant, $\rho\left(v_{i j}\right)$ - Photon mode density, $i$ - Initial state, $j$ - Finial state, $v_{i j}$ - Transition density

A fluorophore located in the close proximity to the metal nanostructure experiences an enhancement in its fluorescence intensity. The fluorescence enhancement occurs due to the increase in population in the excited state and increase in quantum yield of the fluorophore. Increased electric field causes enhanced causes excitation in the fluorescent dye and its directly proportional to the square of intensity of electric field enhancement [58]. The maximum fluorescent enhancement occurs when the wavelength of the dye absorption equals the fluorescent dye absorption wavelength [58]. 
In this case, when the absorption wavelength of the fluorescent dye equals the resonance wavelength at which localized surface plasmon resonance occurs then the maximum fluorescence enhancement occurs. If the dye molecule has very low quantum yield $Q_{0}$; the fluorescence enhancement is very effective. However it's not significant for a dye with high quantum yield [58]. This phenomenon actually increases the photostability and decreases the fluorescence lifetime [67]. However, if the metal-dye distance is within 5nm, a quenching effect occurs [58].

Electric fields can be increased by creating an array of metal nanoparticles, where the electric field is highest between the metal nanoparticles. Hence fluorophores located in between the metal nanoparticles where the electric field is larger, can contribute significantly for metal enhanced fluorescence [9]

\subsubsection{Fluorescence enhancement}

Surface enhanced fluorescence is directly proportional to the field enhancement factor and the modified quantum yield.

$$
\operatorname{SEF} \propto M\left(\lambda_{o}\right) \phi
$$

Equation 2.14

SEF - Surface enhanced fluorescence, $M\left(\lambda_{o}\right)$ - Electric field enhancement factor, $\phi$ - Modified quantum yield of the dye.

The electric field is confined to the surface of the nanoparticle, this is called as the near field enhancement. The electric field enhancement is dependent on the wavelength. Upon excitation of the nanostructure with the resonance wavelength, produces highest near field enhancement. The electric field enhancement factor is directly proportional to the Extinction cross section. The extinction cross section is directly proportional to the wavelength [67].

When the fluorophore is located within the near field enhanced electric field of the surface plasmon, the radiative decay rate of the fluorophore increases. This improves the enhancement of the fluorophore in the vicinity of the metal nanoparticle. 


\subsection{Confocal Microscopy}

Confocal microscopy is a very sensitive method for recording fluorescence images. It consists of a laser which is focused onto a diffraction-limited spot and the surface is raster scanned with respect to $\mathrm{z}$-axis with each $\mathrm{z}$-slice of about 1 micron in size.

The emitted fluorescence signal from the sample is focused through a pinhole then to the detector. The fluorescent image is built from each spot pixel-by-pixel.

Confocal microscope consists of a laser excitation source, which is directed towards pinhole and then towards the beam splitter, where the light is reflected back and focused back on to the sample through objective. The laser is scanned over the surface in the focal plane and the fluorescence signal is emitted back to the detector through the objective and it passes through the emission filter which allows only the wavelengths greater than the particular wavelength [68]. The microscope consists of photomultiplier detector (PMT) which blocks the fluorescence light that is out of focus. The data is later collected by the computer for recording, processing, display and analysis of images. Zen Lite software is used for this purpose. Laser scanning confocal microscope is used to scan the sample and build the fluorescent images.

Optical biosensing techniques have flexibility to be portable, sensitive, simple and easy to use. To test plasmonic nanostructures, $\mathrm{CdSe} / \mathrm{ZnS}$ Quantum dots with wavelength $560 \mathrm{~nm}$ is chosen. Optical biosensor with visible wavelength dye will require less instrumentation to test the biomolecules and hence can be easily portable. It have advantage of multiple detection of biomolecules by attaching labels with different wavelengths to detect various biomolecules.

To test the plasmonic nanostructures $\mathrm{CdSe} / \mathrm{ZnS}$ Quantum dots from nanomaterials and nanofabrication laboratories were used which has the peak excitation wavelength at $560 \mathrm{~nm}$ and peak emission wavelength at $585 \mathrm{~nm}$ as shown in the Figure 2.4. The spectrum below shows the excitation and emission spectra of the $\mathrm{CdSe} / \mathrm{ZnS}$ quantum dots. 


\subsection{Concentration calculation for Quantum dots:}

The quantum dots used for the characterization are $\mathrm{CdSe} / \mathrm{ZnS}$ quantum dots from Nanomaterials and Nanofabrication labs. The molecular weight of the CdSe/ZnS quantum dots is 970,000 . The concentration of quantum dots is $25 \mathrm{mg}$ quantum dots in $5 \mathrm{ml}$ of toluene. The number of dots per

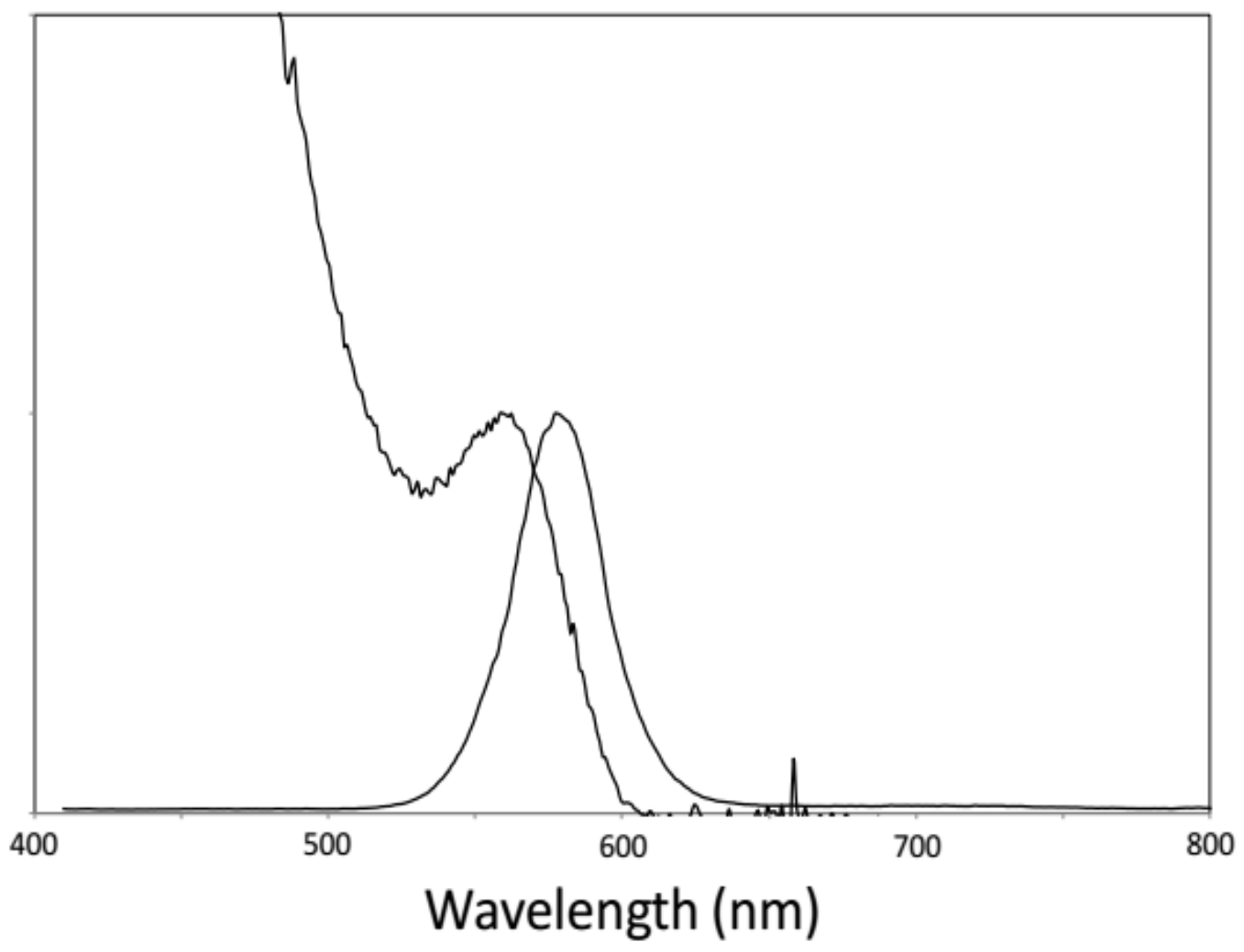

Figure 2-4: Spectrum of CdSe/ ZnS Quantum dots

$\mathrm{ml}$ is $10^{17}$.

Calculation of concentration of quantum dots,

$$
\text { Molar Concentration }=\frac{\text { Number of Moles }}{\text { Volume }}
$$

Equation 2.14 
$\frac{\text { Moles of quantum dots }}{\text { Volume of solution }}=\frac{25 \mathrm{mg}}{970,000} / 5 \mathrm{~mL}=5 \times 10^{-6} \frac{\text { Moles }}{\mathrm{L}}=5 \times 10^{-6} \mathrm{M}$

Original Molar concentration of QD's $=5 \times 10^{-6} \mathrm{M}$

The Original solution is diluted 3 times mixed with toluene for the experiment

$0.5 \mathrm{~mL}$ of the original concentration solution with molar concentration of $5 \times 10^{-6} \mathrm{M}$

is taken and mixed with $1.5 \mathrm{~mL}$ of toluene to make the total volume of the solution to $2 \mathrm{~mL}$,

Calculation of the concentration after dilution from Equation 2.16,

$$
\mathrm{C}_{1} \mathrm{~V}_{1}=\mathrm{C}_{2} \mathrm{~V}_{2}
$$

where $C_{1}$ is the original concentration, $V_{1}$ original volume, $C_{2}$ concentration after dilution, $V_{2}$ volume after dilution

$\left(5 \times 10^{-6} \mathrm{M}\right)(0.5 \mathrm{~mL})=\mathrm{C}_{2}(2 \mathrm{~mL})$

$\mathrm{C}_{2}=1.24 \times 10^{-6} \mathrm{M}$

Hence, the concentration of $1.24 \times 10^{-6} \mathrm{M}$ is used for the experiment (discussed in chapter 5). 


\section{Chapter 3}

\section{SIMULATIONS}

The optical properties of metallic nanoparticles are a subject of considerable interest experimentally in the fields of sensing and medical and solar fields. The optical response of metallic nanoparticles is dominated by their plasmon resonances, which can be defined as collective motion of the conduction electrons. The plasmon resonances occur at specific wavelngths and can be easily identified experimentally as pronounced peaks in the optical absorption spectrum. In general, the plasmon energies depend on the density of conduction

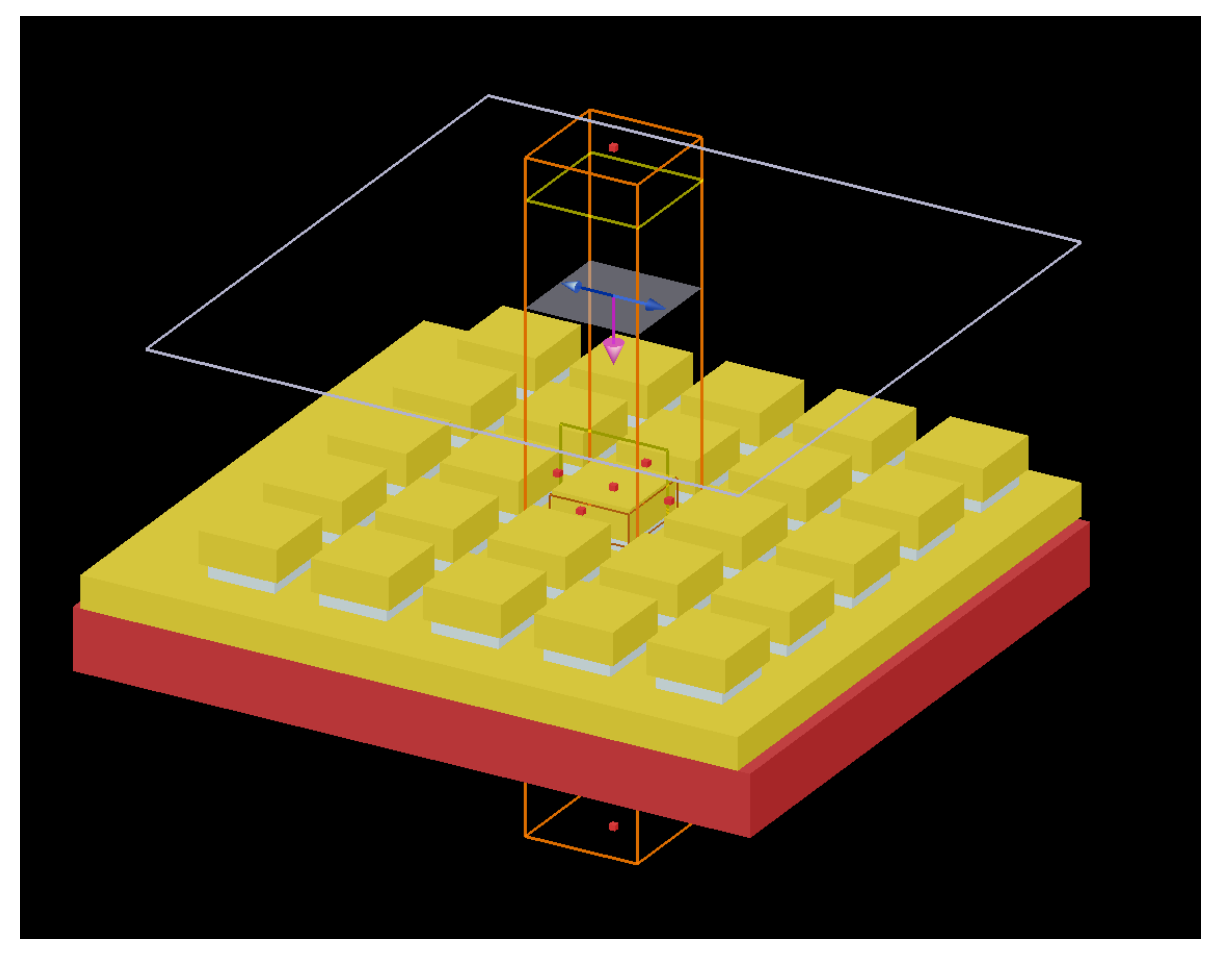

Figure 3-14:

Figure 3-1: Simulation space for plasmonic nanostructure simulation 
electrons and the geometry of the nanoparticle. If the dielectric function of the metallic phase is known, the optical response of the particle can be calculated using the equations of classical electromagnetism.

Finite difference time domain algorithms analyze structures by solving the differential form of coupled Maxwell's equations in time domain. This method involves discretization of the Maxwell's equations in both the time and the space domains in order to find the $\mathrm{E}$ and $\mathrm{H}$ fields at different positions and at different time-steps. They can be used for the analysis (electric and magnetic fields distribution and determination of energy and power distributions, etc.) of metallic nanoparticles, nanorods, nano-apertures substrates and other nanostructures on planar, in liquid media, in or on the surface of waveguides, etc.

The nano-plasmonic phenomenon happens when the permittivity of the particle is a complex function of frequency and its real part of the permittivity is negative in some frequencies, and the free-space wavelength is large in comparison with the particle dimensions; these two conditions only occur in nanoscale [8]. In plasmonic nanosensors, t Lorentz-Mie theory is used to evaluate the extinction energy for small spherical particles.

\subsection{Lumerical}

In this work, FDTD software called Lumerical is used to carry out FDTD analysis for the plasmonic nanostructures. This software enables FDTD analysis of the metallic media using the dispersion model - Johnson and Christy model for determining the dielectric constant for gold and palik reference for silicon. The more detail steps of the working of Lumerical are discussed in Appendix B.

Electromagnetic fields (i.e., $\mathrm{E}$ and $\mathrm{H}$ fields in the $\mathrm{x}, \mathrm{y}$, and $\mathrm{z}$ directions) in the vicinity of the metallic nanostructures were calculated assuming plane wave illumination, with wavelengths varying between $400 \mathrm{~nm}$ and $700 \mathrm{~nm}$. The magnitude of the incident electric fields was taken to be unity and the enhancement of electromagnetic fields, around the gold nanostructures is evaluated. Tuning the plasmon or extinction spectrum is the main concern in designing nanoplasmoinc devices. Nano-particle geometries and incident wave attributes are important for 
careful design of extinction spectrum. The goal of this simulation effort was to understand the different parameters that influence the LSPR extinction spectrum. The simulated nanostructure is shown in the Figure 3.1. The calculated electric field intensity around the nanostructure is shown in the Figure 3.2.
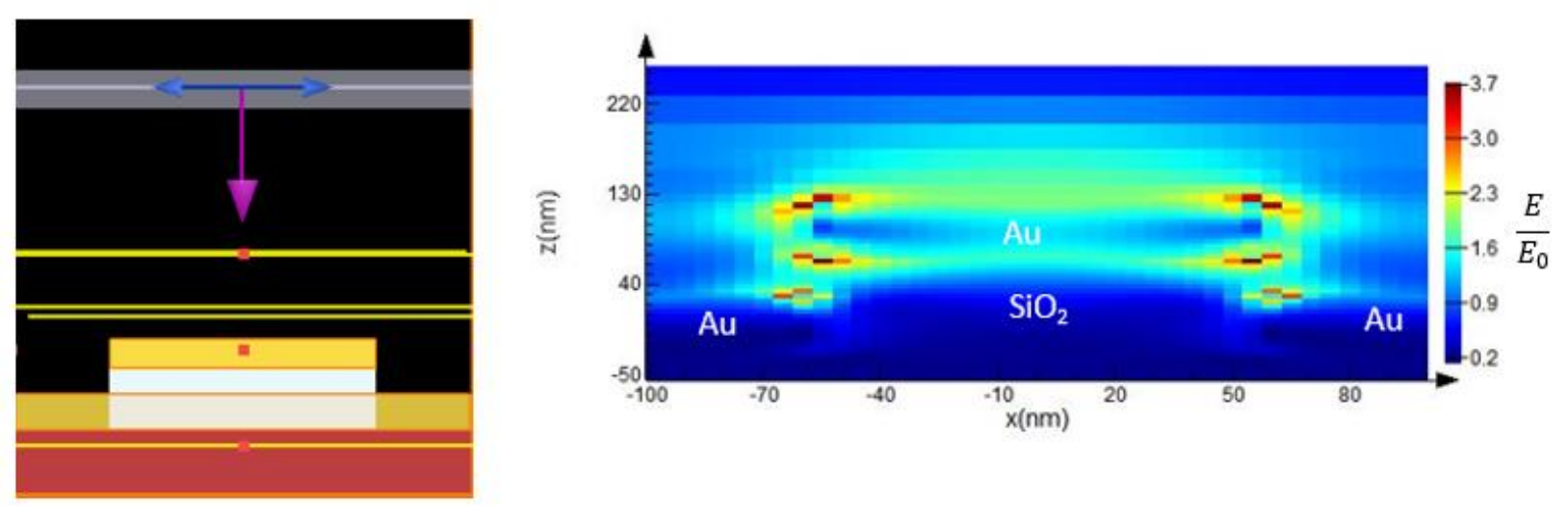

Figure 3-2: Electric field intensity enhancement around the nanostructure

\subsection{Localized surface plasmon resonance (LSPR) Design Parameters:}

Tuning LSPR parameters is important for maximum sensitivity and accuracy. In this section, the most important parameters affecting the plasmon spectrum are described, providing knowledge required to design and fabricate a LSPR device suitable for biosensing applications.

LSPR nanosensors work based on the fact that the metal nanoparticles have strong extinction spectrum. Extinction spectrum is the sum of rayeligh scattering and absorption. The collective oscillation of conduction band electrons results in extinction peak. The extinction peak depends on the physical parameters like type of the material, shape and size of the material. The extinction peak defines the electric field enhancement around the nanostructure. 


\subsubsection{LSPR Design Parameters, Material Effect}

Typically a metal is considered as a solid material with many free outer band electrons, which respond to the incident electric field, so the incident electric field inside the metal is considered to be zero. This actually makes the metal to be perfectly reflective to the incident light (electromagnetic field). Typically from this, the conductivity of the metal is assumed to be infinite. But upon observation of the frequency response of the metal to the incident electromagnetic radiation, some of the waves become attenuated. The waves become attenuated above a particular angular frequency which is referred as plasma frequency $\left(\omega_{\mathrm{p}}\right)$, above which the electron gas is transparent to the incident field allowing it to pass through the metal. Above the plasma frequency, the fields oscillate too fast that the metal loses its reflectivity. Typically for the metals the plasma frequency is in deep UV region except for gold and silver. Light below the plasma frequency is reflected and light frequencies above the plasma frequency are transmitted. As the electrons cannot screen so fast above the plasma frequency, hence they are transmitted. Gold and copper have inter-band transitions in the visible region, where specific wavelengths of light is absorbed which gives out a different color.

Every metal cannot exhibit plasmonic properties but exhibit metallic behavior. Most of the times, the plasmonic behavior, and plasma frequency is observed in ultraviolet region. Only few metals exhibit plasmonic frequency in visible region like silver, gold and copper. The most common metals used in LSPR applications are silver $(\mathrm{Ag})$, gold $(\mathrm{Au})$, copper $(\mathrm{Cu})$, and aluminum $(\mathrm{Al})$. Silver and gold are more common than the others because of their high enhancement capability they are mostly used for plasmonic application. Copper is typically suitable for longer wavelengths usually greater than 600nm. But copper and silver have probability of high oxidization. Gold has good stability in atmospheric conditions and good biocompatibility.

The plasmonic phenomenon occurs only in the reflection mode, where the nanostructures rather than reflecting the light they scatter the light. Silver is reflective in visible region, whereas gold is reflective at wavelengths greater than $520 \mathrm{~nm}$. 
The LSPR resonance frequency depends on the real part of the permittivity constant of the metal, and resonance line width. Thus, the smaller the real part of the permittivity is, the higher the plasmon frequency becomes.

\subsection{LSPR Design Parameters, Size Effect}

For higher scattering rate, the metal feature size must be much smaller than the excitation wavelength of light. Typically the excitation wavelength is $500 \mathrm{~nm}$ and the size of the nanostructures typically vary from 5nm - 100nm [69].

The nanoparticle size has two different impacts on the generation of plasmons

(i) If the nanoparticle is small enough (less than $20 \mathrm{~nm}$ for gold), the intrinsic effect of the particle directly affects the metal permittivity.

(ii) Extinction peak variation with refractive index If the particle is not small enough (larger than $25 \mathrm{~nm}$ for gold), the extrinsic effect is enabled, where the extinction coefficient is dependent on the size $r$.

\subsubsection{LSPR design parameters, effect of refractive index}

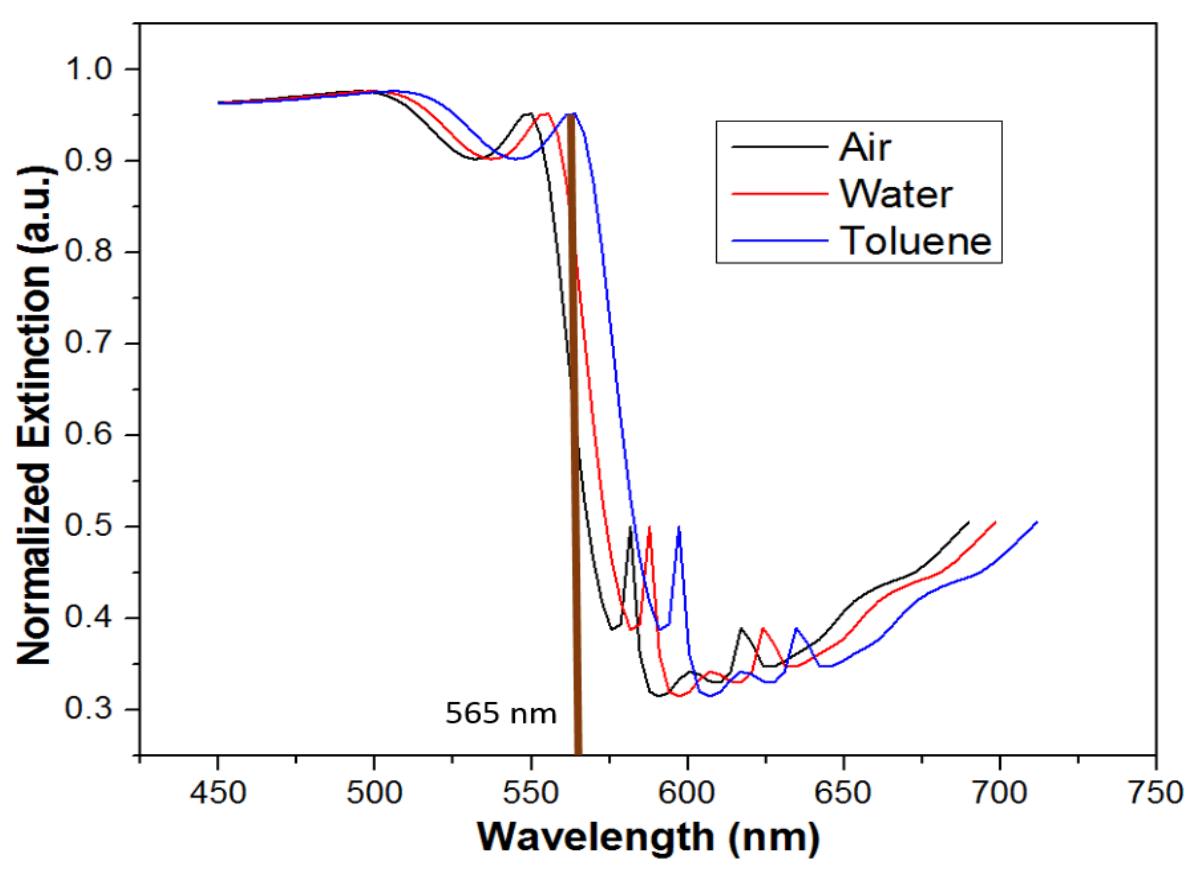

Figure 3-3: Extinction peak variation with refractive index 
The extinction peak is shifted towards right with increase in refractive index of the background. This sensitivity of plasmon resonance peak to the refractive index is sued as the basis for label free detection of biomolecules.

\subsubsection{LSPR design parameters, spacing effect}

The potential application of electron beam lithography is that an array of nanoparticles can be fabricated. As part of the design, upon gold deposition on top of the dielectric nanopillars, creates an array of gold nanoparticles. This array creates a sum of the potential energies of the generated single plasmons of each nanoparticle. This gap produces "hot spots", where surface
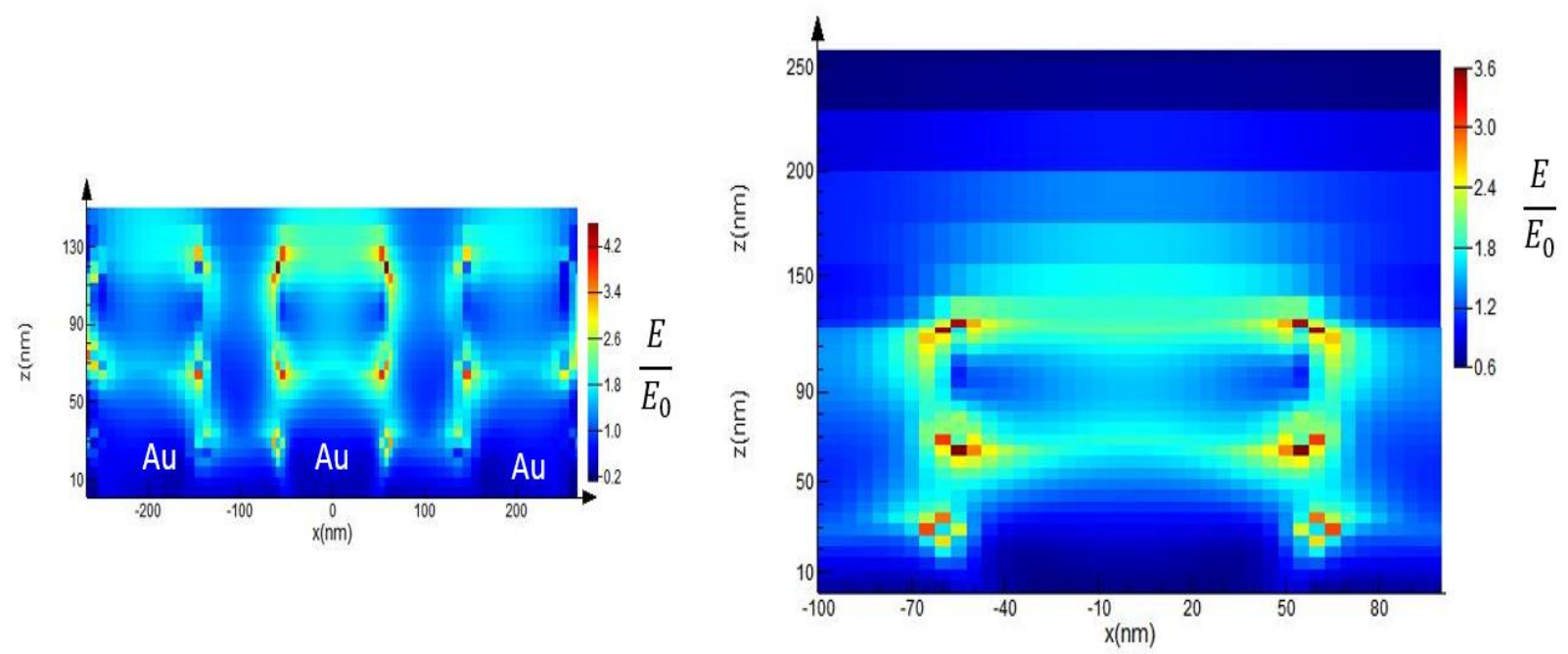

Figure 3-4: Electric field intensity indicating hotspots in between the gold nanopillars

plasmons interact mutually, and thus, provide the hottest point of the plasmon signal. Therefore, if a target molecule trapped in these hot spots, it will generate the highest fluorescence enhancement signal. 
The single plane gold surface, the extinction spectrum peak has been increased 3 fold compared to the plain gold layer as shown in the Figure 3.5. The main reason for increase in extinction coefficient in multiple particles gold nanoparticles as compared with the plain thin film of gold is due to two factors, firstly due to localizing the potential to a confined region due to the presence of multiple nanoparticles and secondly, the coupling of the singlet resonances in each particle, and large interparticle coupling effects can be achieved in LSPR substrates fabricated with electron-beam lithography.

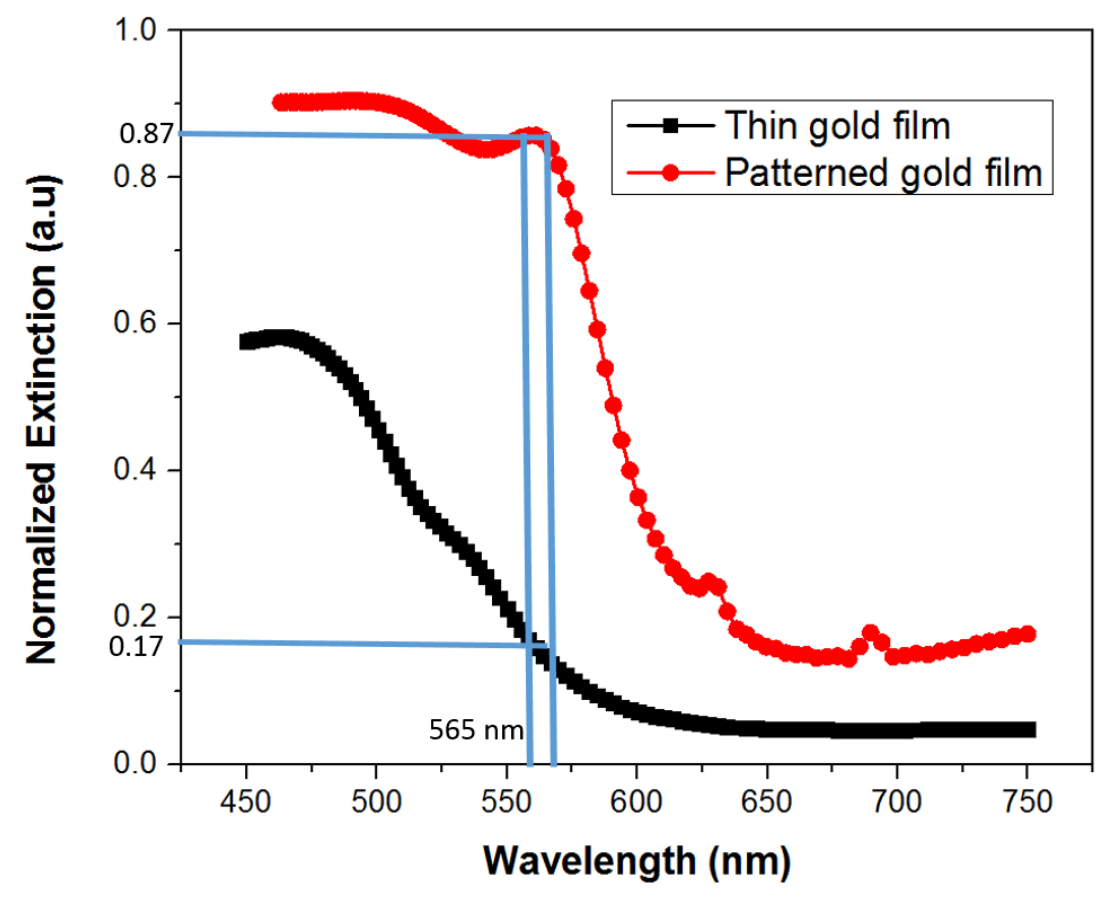

Figure 3-5: Extinction peak variation thin gold film vs patterned gold film 
It can be seen that the maximum enhancement occurring between wavelengths $546 \mathrm{~nm}-560 \mathrm{~nm}$, increases with decreasing the spacing as shown in Figure 3.6.

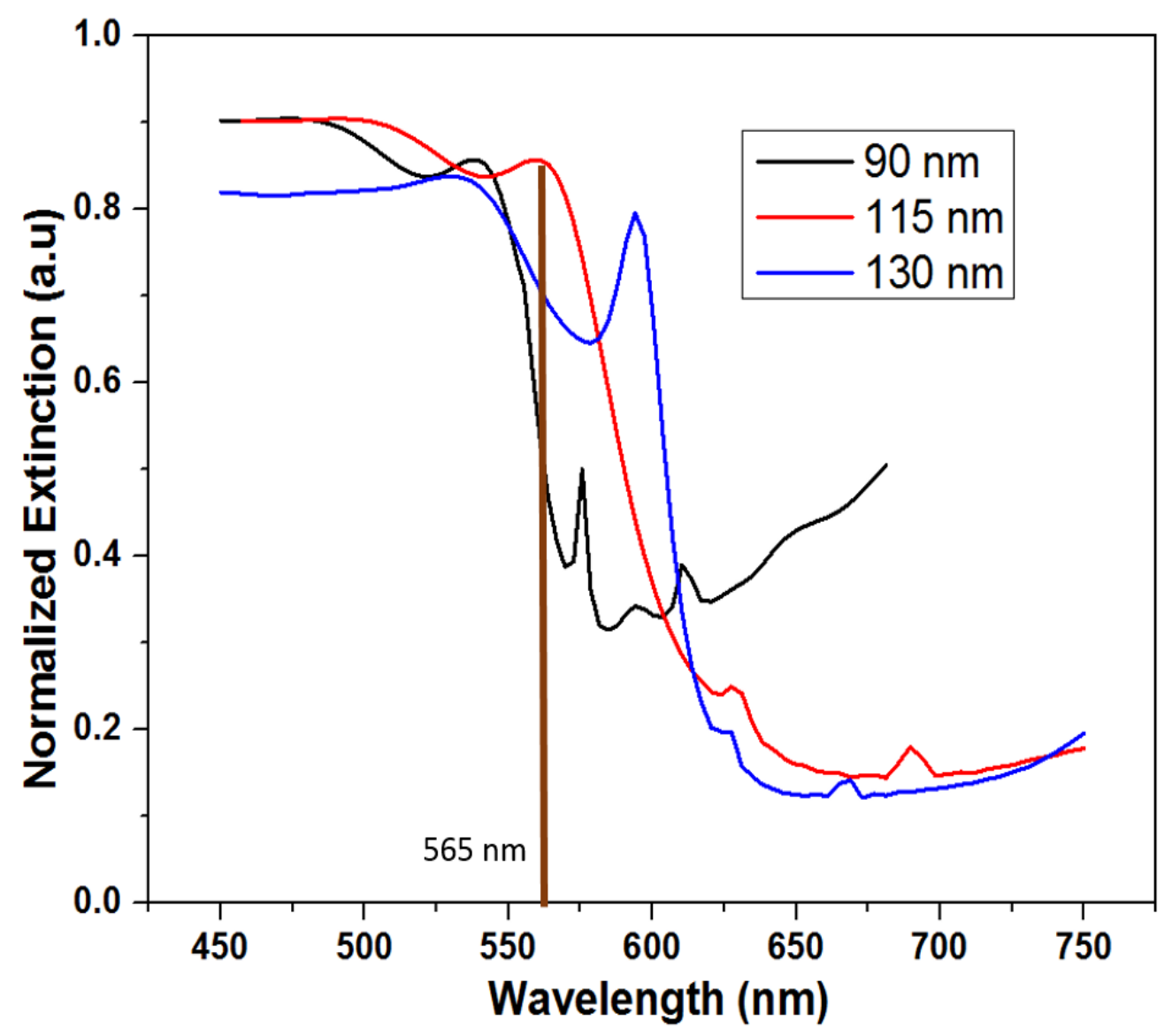

Figure 3-6: Extinction peak variation with diameter of the nanoparticles 


\subsubsection{LSPR Design Parameters, thickness of gold deposition}

The simulations shows that the increase in gold deposition leads to red shift in the localized surface plasmon peak as shown in the Figure 3.7, this is due to the increase in lateral size of the pillars which red shifts the surface plasmon peak. Gold deposition of $60 \mathrm{~nm}$ is having highest electric field enhancement at $565 \mathrm{~nm}$ wavelength. So, gold deposition of $60 \mathrm{~nm}$ is chosen for fabrication.

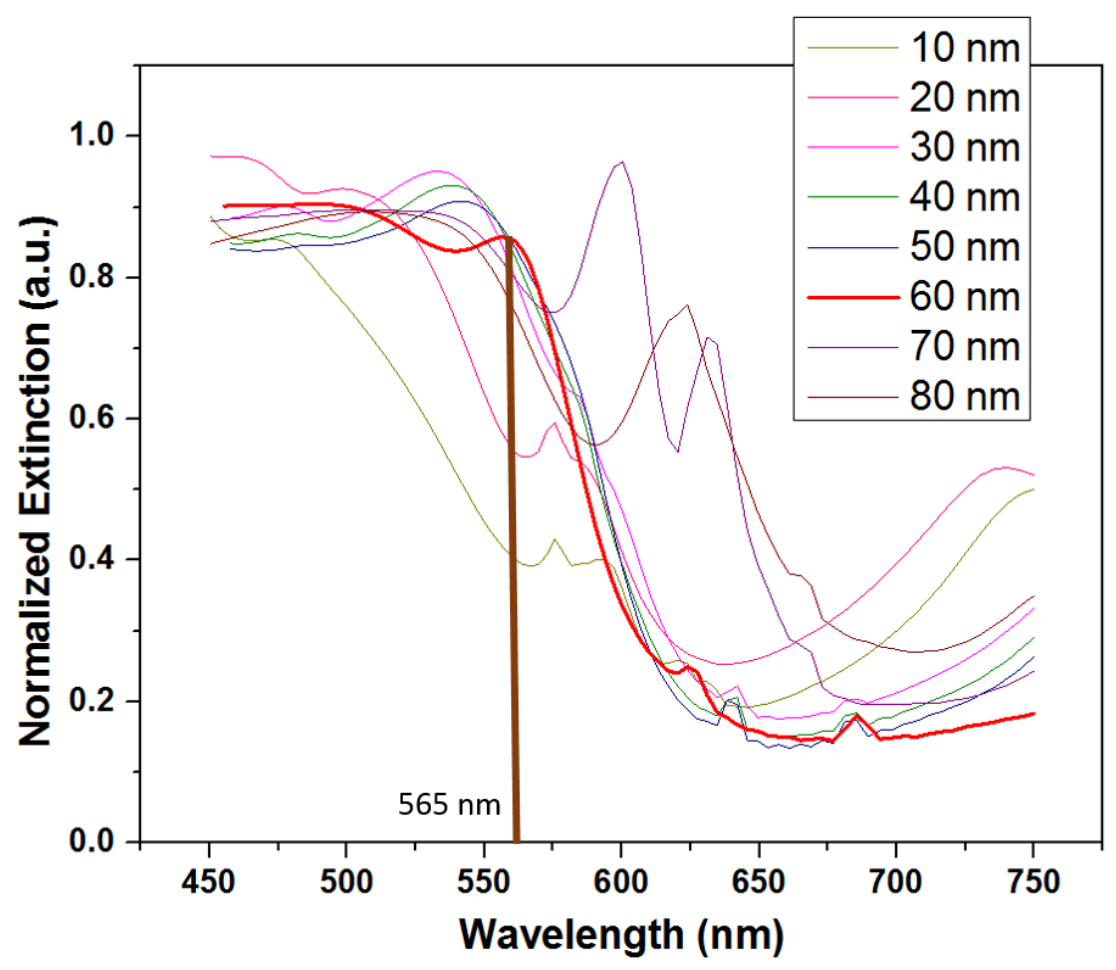

Figure 3-7: Extinction peak variation with gold deposition thickness

\subsection{CONCLUSION}

In this chapter, the effects of morphological and electromagnetic parameters on the LSPR extinction spectrum are formulated based the simulation using FDTD software. The required sensitivity and electromagnetic field enhancement variation with the spacing and thickness of 
gold deposition were modeled. Finally the paramters were modeled for high enhancement in electric field around the nanostructures

The following table gives the list of parameters modeled crystals and the tolerance to the fabrication. The parameters taken form the simulation are used for real time fabrication and testing of plasmonic nanostructures.

Refractive index maintained at 1.4 for toluene, Table 3.1 gives the list of modeled parameters and tolerance to the fabrication.

Table 3-1: Table showing the modeled parameter and the tolerance for the fabrication

\begin{tabular}{|l|l|l|}
\hline Parameter & Modeled Value & Tolerance \\
\hline Lattice Constant & $200 \mathrm{~nm}$ & $+/-5 \mathrm{~nm}$ \\
\hline Radius of $\mathrm{SiO}_{2}$ Nanopillars & $115 \mathrm{~nm}$ & $+/-5 \mathrm{~nm}$ \\
\hline Thickness of gold deposition & $60 \mathrm{~nm}$ & $+/-2 \mathrm{~nm}$ \\
\hline
\end{tabular}




\section{Chapter 4}

\section{FABRICATION}

This chapter includes the fabrication process of plasmonic nanostructures. The fabricated structure includes 2D periodic square $\mathrm{SiO}_{2}$ nanopillars fabricated by e-beam lithography then followed by gold deposition and thin layer of dielectric spacer layer. This chapter also includes high resolution scanning electron microscopy images of the nanostructures.

\subsection{Summary of process}

Table 4-1: Summary of the process: Fabrication of plasmonic nanopillars

\begin{tabular}{|l|l|l|}
\hline Process step & Pictorial version & Process \\
\hline 1. Cleaning & & $\begin{array}{l}\text { Cleaning process is carried out } \\
\text { using Sonication for: 5min in } \\
\text { Acetone, 5min in Methonol, } \\
\text { min rinsing with flowing water } \\
\text { Drying out with Nitrogen gun }\end{array}$ \\
& Silicon Substrate & \\
\hline
\end{tabular}




\begin{tabular}{|l|l|l|}
\hline Process step & Pictorial version & Process \\
\hline $\begin{array}{l}\text { 2. Spinning of e- } \\
\text { beam resist }\end{array}$ & $\begin{array}{l}\text { Silicon Substrate } \\
\text { 3. Electron beam }\end{array}$ & $\begin{array}{l}\text { lithography } \\
\text { of 1200rpm, acl of 13(1729/ } \\
1728) \text { for 1 min. }\end{array}$ \\
\hline $\begin{array}{l}\text { Oxygen } \\
\text { plasma Asher }\end{array}$ & Silicon Substrate & $\begin{array}{l}\text { Spinned XR1541 resist is } \\
\text { subjected to electron beam } \\
\text { lithography using the pattern } \\
\text { generated using design CAD file }\end{array}$ \\
\hline $\begin{array}{l}\text { 5.Gold } \\
\text { deposition }\end{array}$ & Silicon Substrate & $\begin{array}{l}\text { Oxygen plasma ashing is done } \\
\text { at power of 300milli watts, time } \\
\text { for 10 min and @ pressure of } \\
300 \text { torr }\end{array}$ \\
\hline
\end{tabular}




\begin{tabular}{|l|l|l|}
\hline Process step & Pictorial version & Process \\
\hline $\begin{array}{l}\text { 6. Spacer layer } \\
\text { deposition }\end{array}$ & Silicon Substrate & $\begin{array}{l}\text { Using Temescal Ebeam } \\
\text { evaporator, thin 10nm layer of } \\
\mathrm{SiO}_{2} \text { is deposited with } \\
\text { deposition rate of } 0.2 \mathrm{~A} / \mathrm{s}\end{array}$ \\
\hline
\end{tabular}

\subsection{Fabrication process}

The structure of the fabricated plasmonic nanostructure is shown in Figure 4.1. The fabrication

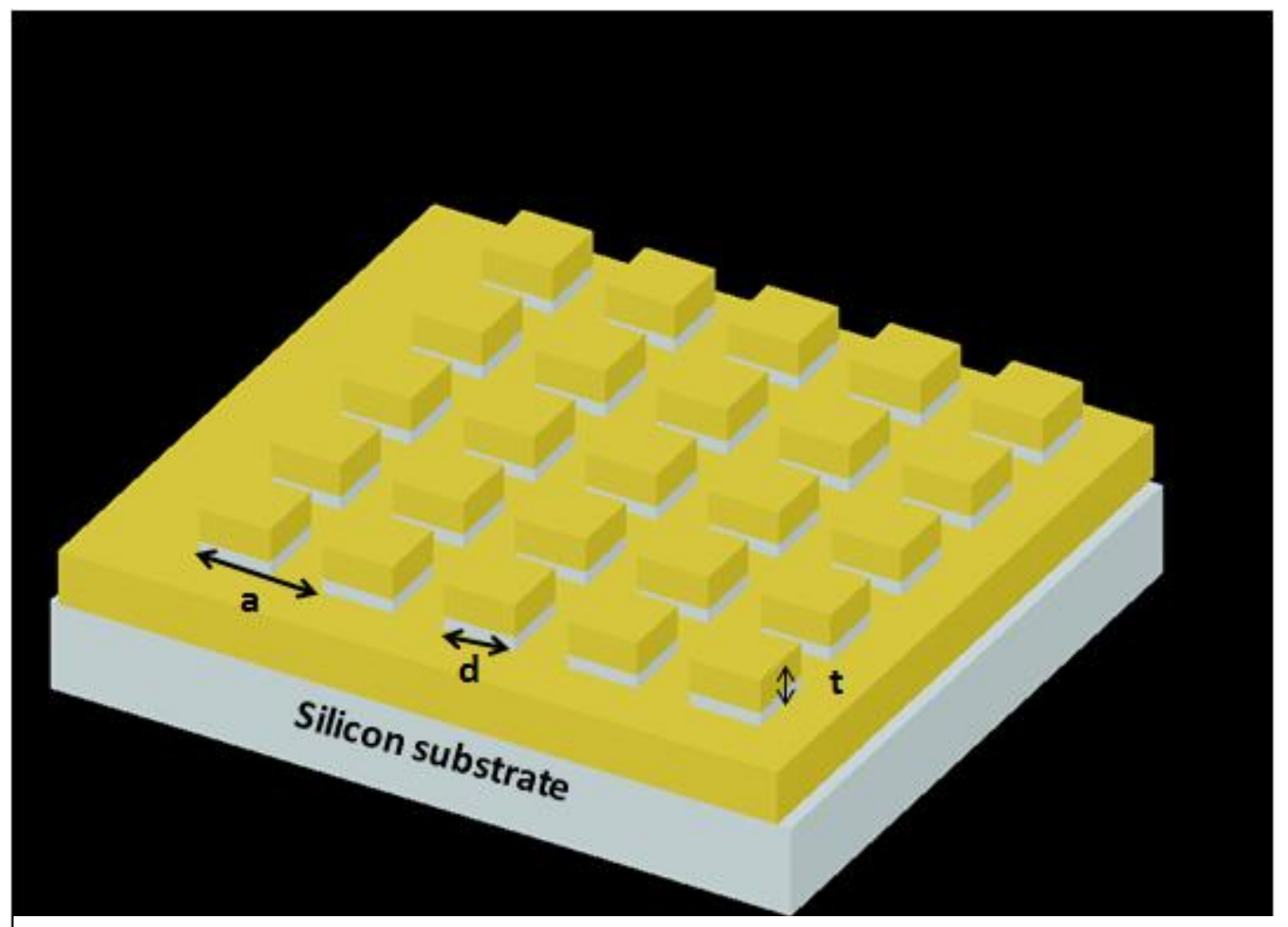

Figure 4-1: Fabricated square plasmonic gold nanopillars on silicon substrate 
of plasmonic nanostructures is carried out in class 100 and class 1000 cleanroom environments. A 6 inch silicon wafer (100) is cut into small $(1 \mathrm{~cm} \times 1 \mathrm{~cm})$ square samples and these samples are used for fabrication.

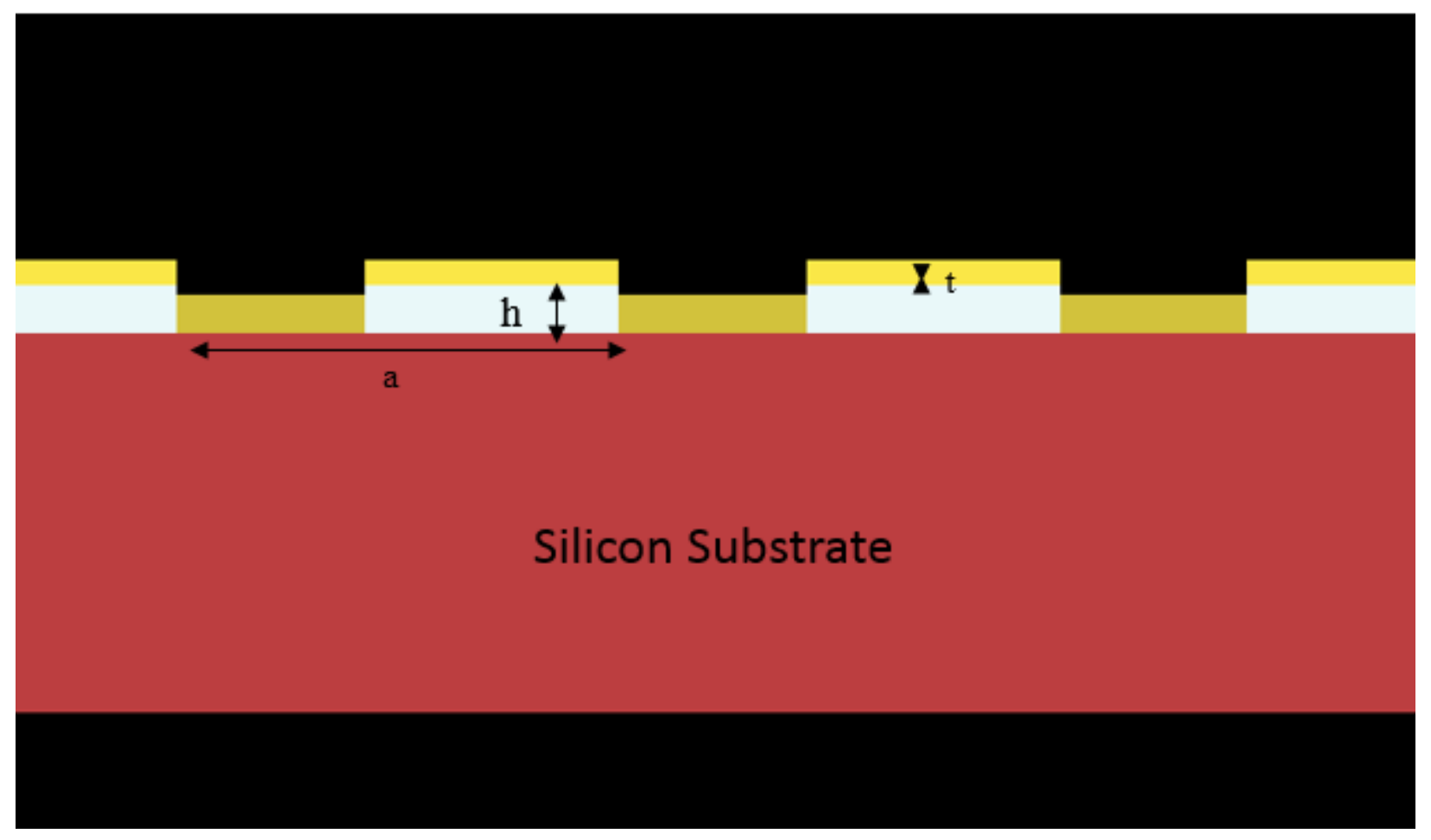

Figure 4-2: Side view of the fabricated plasmonic nanostructure

\subsubsection{Sample Cleaning}

The sample is cleaned by sonication for $5 \mathrm{~min}$ in acetone, followed by $5 \mathrm{~min}$ sonication for $5 \mathrm{~min}$ in methanol. This process removes organic impurities and dirt from the silicon samples and then the samples are then cleaned with flowing water for 2 min then dried with the nitrogen gun.

\subsection{Nanopillar Fabrication}

The Figure 4.1 and Figure 4.2 shows fabricated square plasmonic gold nanopillars, where a, d, h and $t$ indicate the periodicity, diameter of pillars, height of pillars and the metal deposition thickness, respectively. The parameters of the nanostructure are properly chosen to design a 
plasmonic resonance peak at $\sim 560 \mathrm{~nm}$. The fabrication process of $2 \mathrm{D}$ arrangement of gold square nanopillars in square lattice involved three main processing steps and each one required an optimization and check process.

The first step is the optimization of the e-beam writing process on silicon substrate. To define the square geometry for the 2D pattern, e-beam resist XR1541 is used which is a negative resist. The fabrication of plasmonic nanostructures is carried out using EBL (electron beam lithography). Electron beam lithography allows fabrication of nanostructures with high resolution, and reproducibility. High density nanopattern features are required in order for plasmonic nanostructure exhibiting the desired functions.

\subsubsection{Sample preparation - Spinning of resist}

100nm thickness of XR 1541 is spin coated on silicon substrate to fabricate nanopillars, the resist is spun at speed 1200rpm for 60sec acceleration of 13 (1728/1729) to achieve the thickness of about $100 \mathrm{~nm}$.

\subsubsection{Electron beam Lithography}

Top to down fabrication process is implemented with electron beam lithography through which excellent control of dimensions and the shape of the nanostructures with high resolution can be achieved by electron beam lithography [70, 71]. The high density $2 \mathrm{D}$ periodic $\mathrm{SiO}_{2}$ square nanopillars on silicon substrate are fabricated by electron beam lithography. The electron beam lithography is based on the principle that the e-beam resist polymers are sensitive to the electrons and it can be patterned by the beam of electrons. In this process, e-beam resist XR1541 is used for writing 2D periodic nanostructures using JEOL 2600 SEM equipped with nanopattern generating system (NPGS) software with acceleration voltage of $30 \mathrm{KV}$ and beam current of 40pA. 


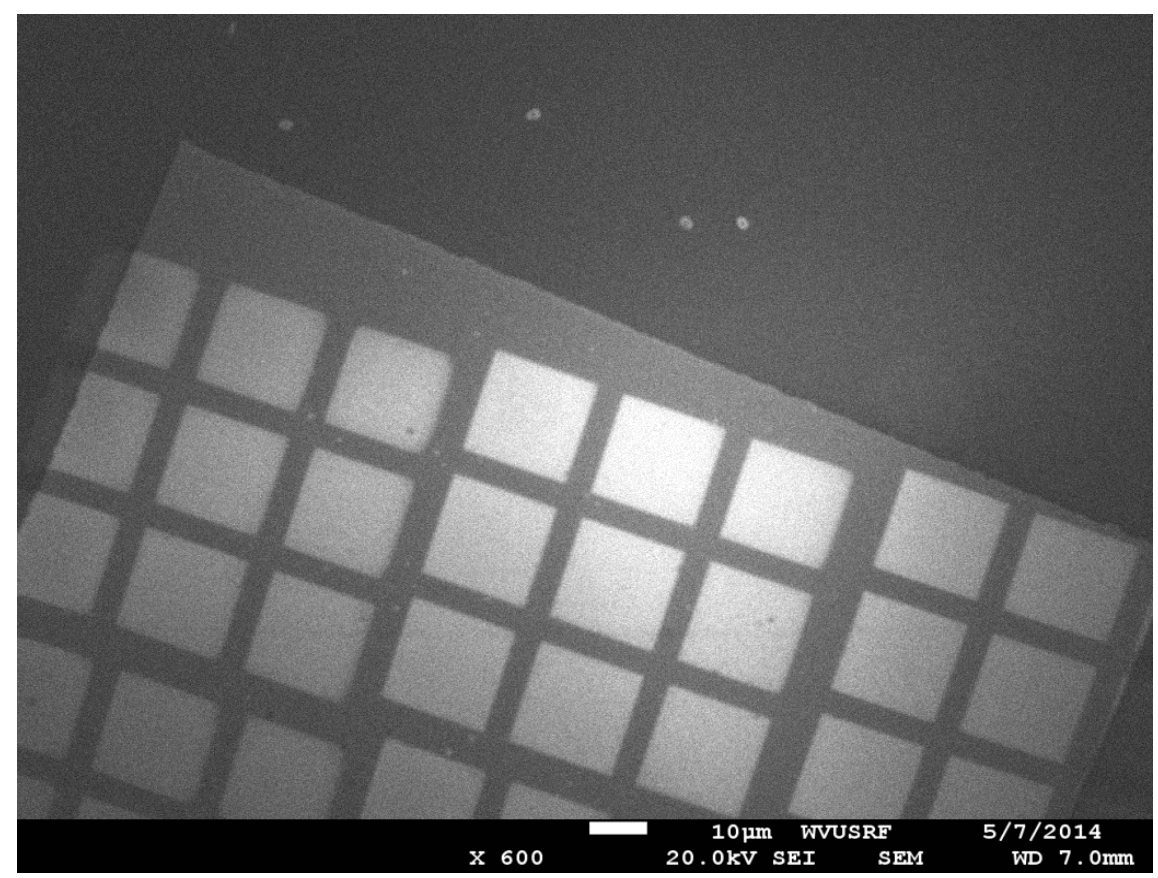

Figure 4-3: Fabricated square nanostructures with varying dosages

In this work, $100 \mathrm{~nm}$ nanopillars are fabricated in a total area of $20 \mu \mathrm{m} \times 20 \mu \mathrm{m}$ with varying 9 different dosages from $295 \mu \mathrm{C}$ to $340 \mu \mathrm{C}$, with each dosage area of $5 \mu \mathrm{m}$ as shown in the Figure 4-3. An array of 4 similar patterns with $5 \mu \mathrm{m}$ spacing between them are fabricated on silicon substrate. Leading to the total fabricated area of $100 \mu \mathrm{m} \mathrm{X} 100 \mu \mathrm{m}$.

The e-beam resist, XR1541 is comprised of hydrogen silsesquioxane resin in a methylisobutylketone (MIBK) solvent. It's a negative tone resist. The electron beam resist which is spun-on the silicon substrate and is used to fabricate dense $100 \mathrm{~nm} \mathrm{SiO}_{2}$ nanopillars. Subsequently the sample was developed in MFCD26A solution and then rinsed with water and dried with nitrogen gun. The fabricated nanostructures are analyzed using scanning electron microscopy (SEM).

\subsubsection{Development}

Trimethyl ammonium hydroxide (MFCD 26A) is used as developer. Development is carried out at $80^{\circ} \mathrm{C}$ in a petri dish for $2 \frac{1}{2} \mathrm{~min}$. 


\subsubsection{HSQ Curing}

XR 1541 upon oxygen plasma ashing converts into $\mathrm{SiO}_{\mathrm{x}}$. HSQ curing is carried under the following conditions.

Figures 4.4 and 4.5 show the fabricated square $\mathrm{SiO}_{2}$ nanopillars on silicon substrate. These pictures are taken using high resolution SEM at the magnification of $25000 \mathrm{X}$ and $90000 \mathrm{X}$ respectively.

\begin{tabular}{|c|c|}
\hline Parameter & Value \\
\hline Pressure & 300 millitorr \\
\hline RF power & 300 Watts \\
\hline Time & 60 sec \\
\hline DC bias & 5 \\
\hline Base pressure & 80 \\
\hline Gas flow & $\sim 47$ (Depends on the time and gas flow \\
& pressure) \\
\hline
\end{tabular}




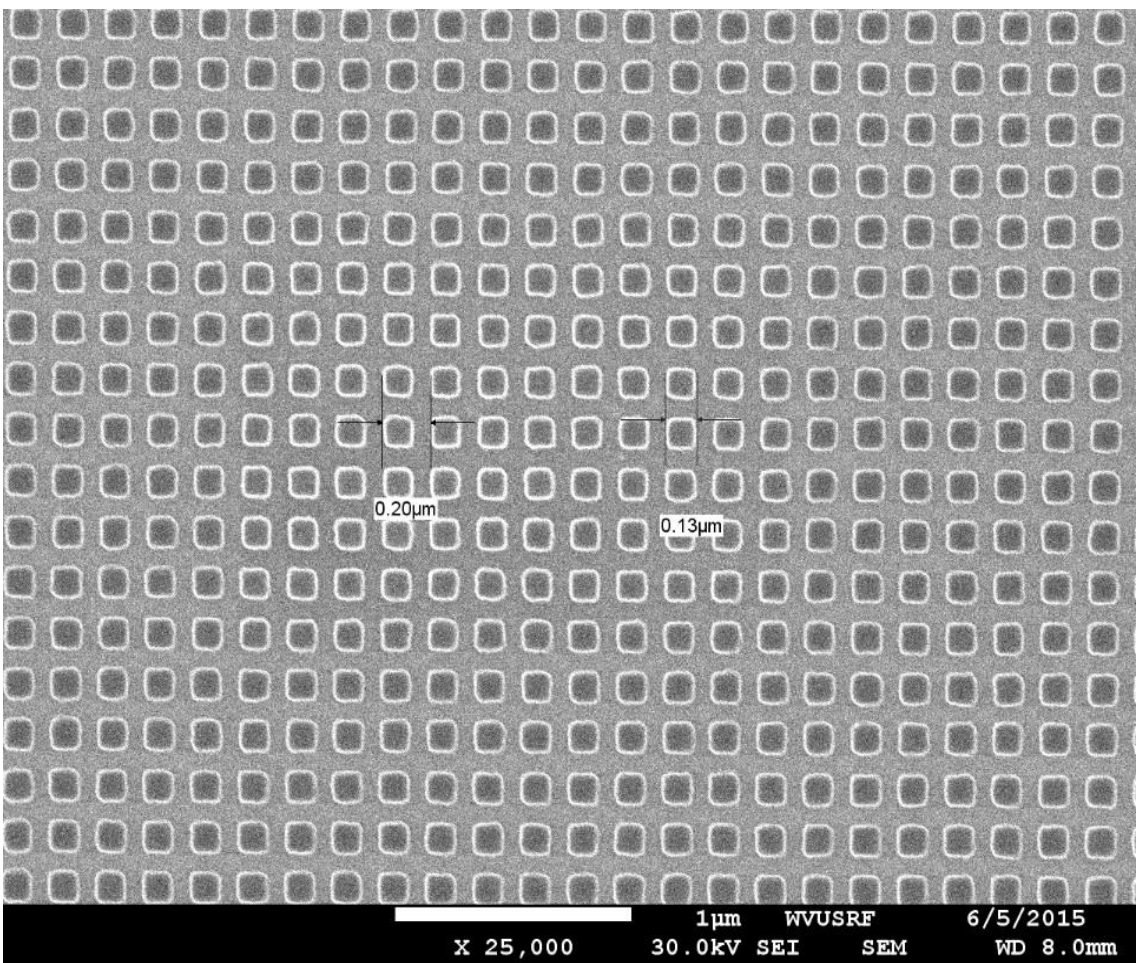

Figure 4-5: Fabricated square nanostructures on silicon substrate using e-beam lithography

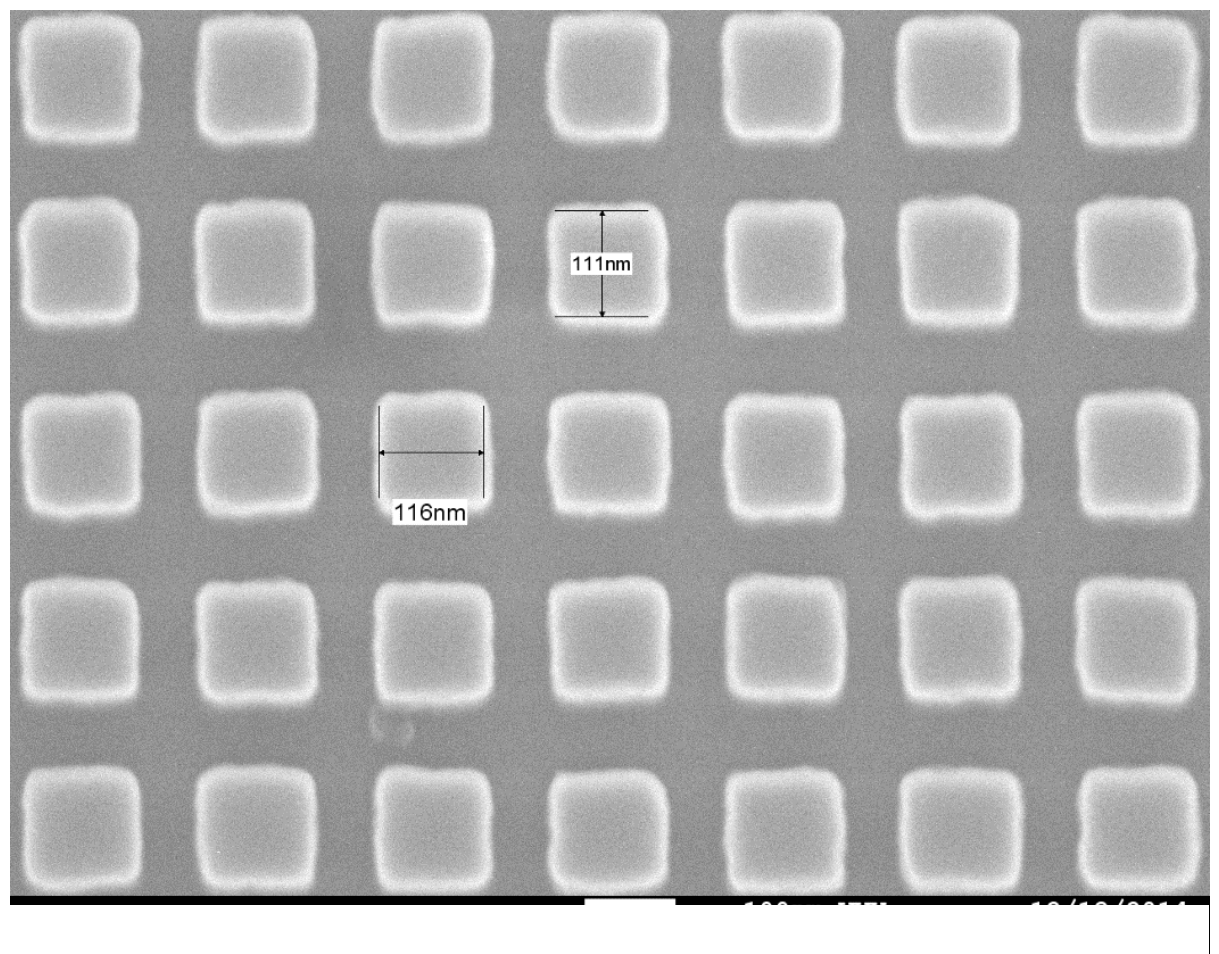

Figure 4-4: SEM image of square nanostructures at magnification of $90,000 \mathrm{X}$ 


\subsection{Gold deposition}

Metal deposition is carried out using physical vapor deposition method, e- beam evaporation using Kurt J Leskar e-beam evaporator in class 1000 cleanroom environment. Gold is deposited on top of thin titanium layer, which acts as adhesion layer. Titanium is deposited at $0.2 \mathrm{~A} / \mathrm{s}$ deposition rate and gold is deposited at $0.5 \mathrm{~A} / \mathrm{s}$ and to achieve thin smooth film. E-beam is used as heat source and the deposition power percentage of $27.4 \%$ for deposition of gold and $6.7 \%$ deposition power percentage for deposition of titanium. The process is carried out under the vacuum of $8 \times 10^{-8}$ Torr. $5 \mathrm{~nm}$ thick titanium is used as adhesion layer between $\mathrm{SiO}_{2}$ and gold. $60 \mathrm{~nm}$ gold is evaporated by using e-beam evaporator. It took about an hour to deposit the metals gold and titanium at the deposition rate of $0.5 \mathrm{~A} / \mathrm{s}$ and $0.2 \mathrm{~A} / \mathrm{s}$.

\subsubsection{Optimization of gold deposition}

Gold is deposited at the deposition rate of 2.0A/s. But, gold deposited with the high deposition rate had a problem of non uniform distribution and stability on the top of pillar. As shown in Figure 4.7. The picture is high resolution SEM image of the fabricated plasmonic structure taken at angle of $70^{\circ}$

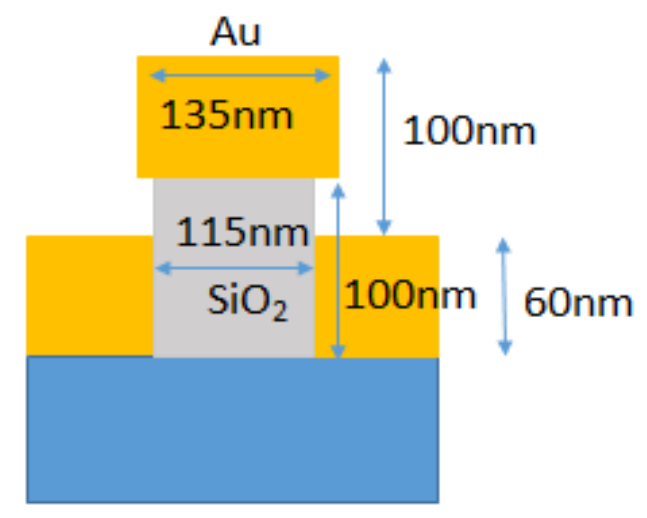

Figure 4-6: Designed parameters for gold deposition on top of nanopillars 


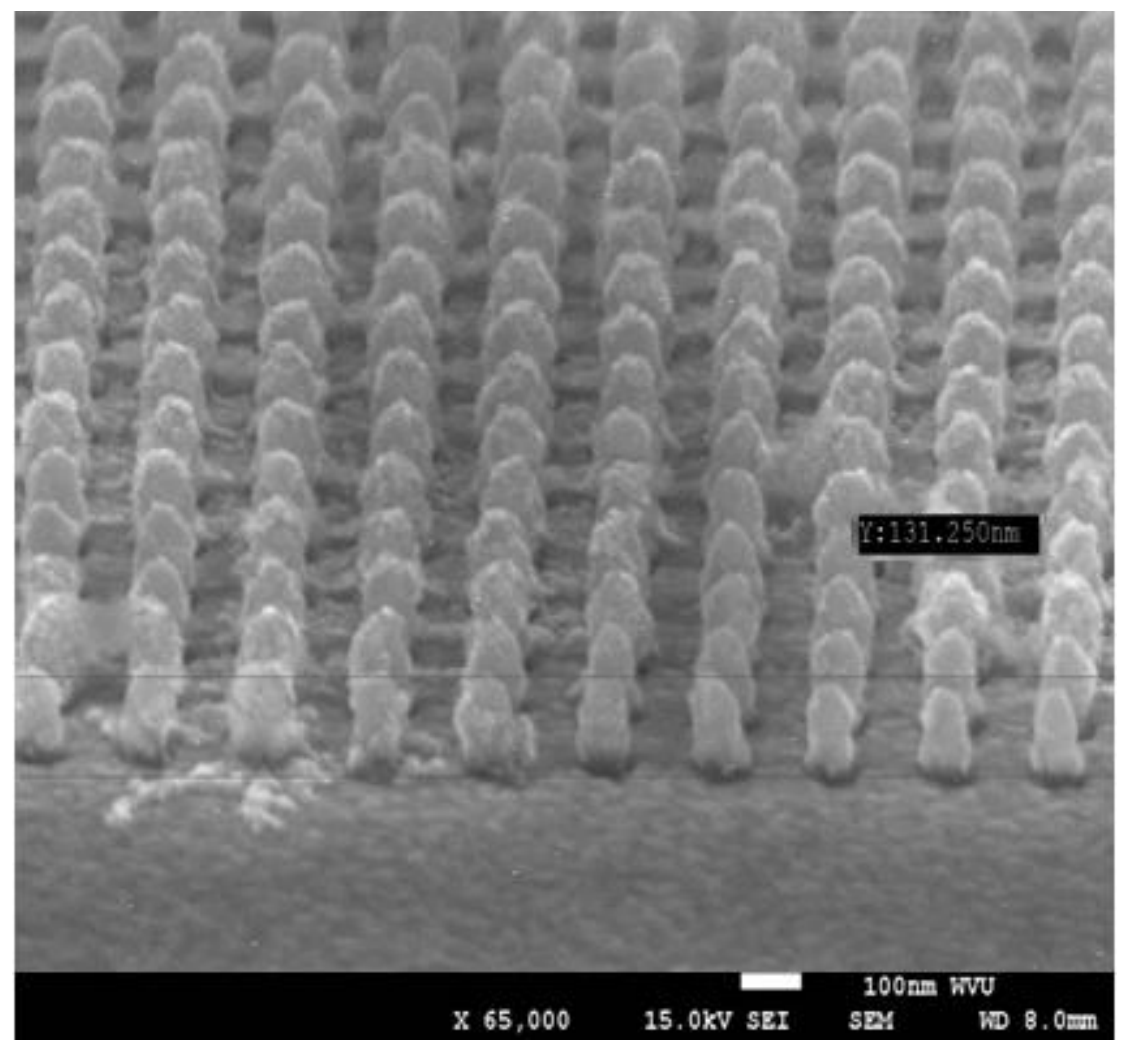

Figure 4-7: SEM picture showing gold deposition with deposition rate of $2.0 \mathrm{~A} / \mathrm{s}$

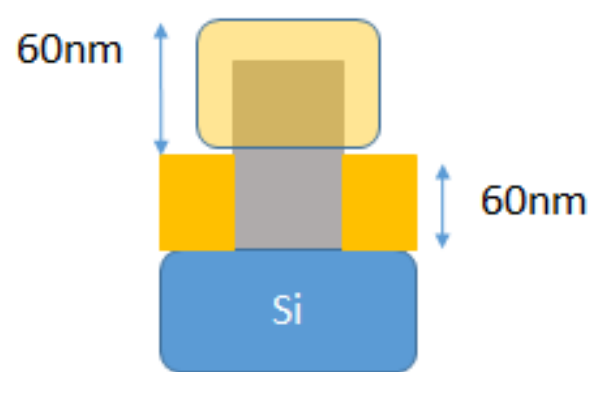

Figure 4-8: Measured values of gold deposition thickness with deposition rate of $2.0 \mathrm{~A} / \mathrm{s}$ 
Upon calculations, as shown in Figure 4.8, the deposited gold thickness measurements on the top of nanopillars did not match the designed simulated parameters as shown in Figure 4.6.

To optimize the gold deposition according to the simulated parameters, deposition rate is decreased and is maintained at $0.5 \mathrm{~A} / \mathrm{s}$. The Figure 4.9 is the top view SEM image of the gold deposited on the top on square nanopillars.

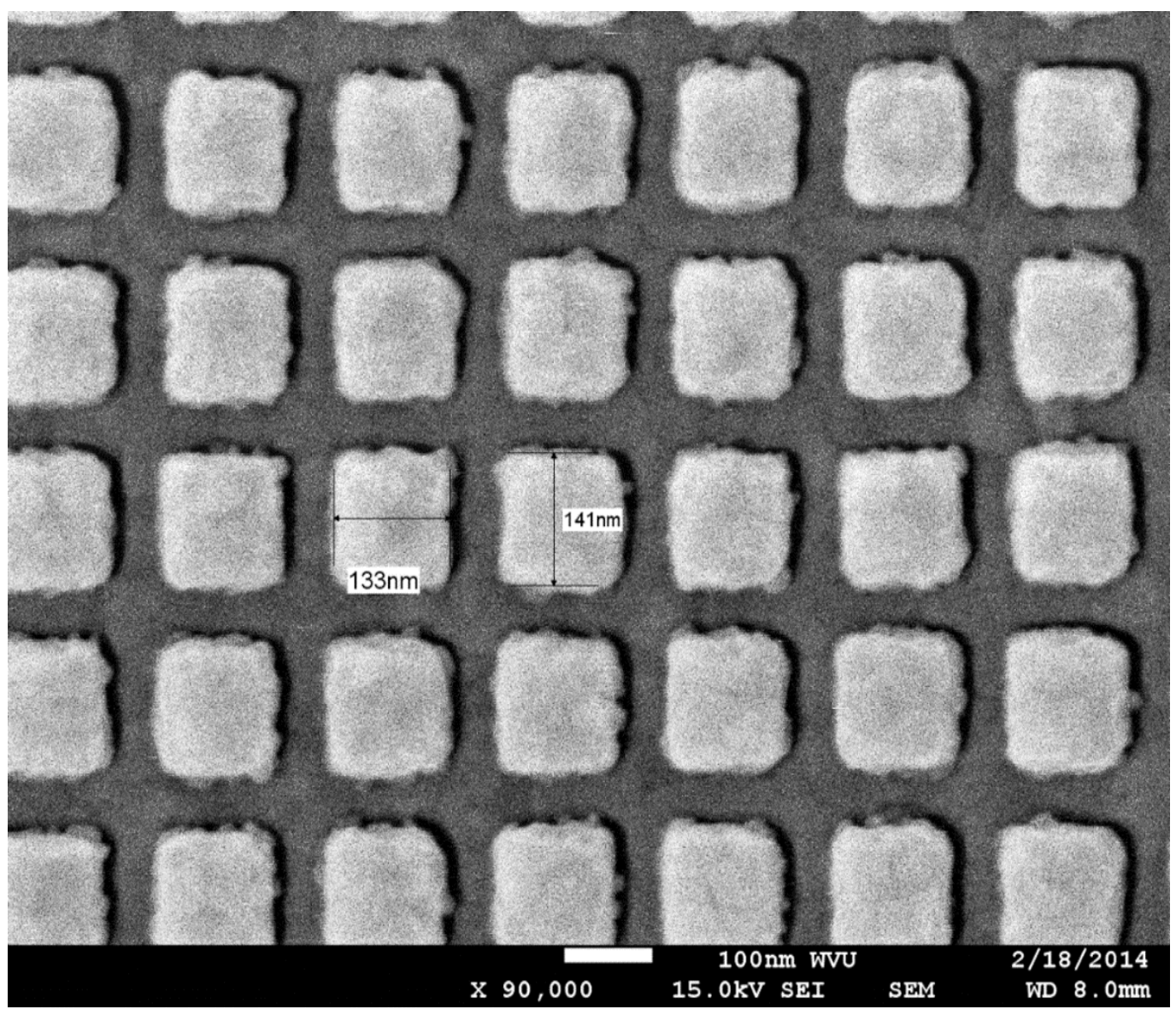

Figure 4-9: SEM image of Gold deposition on top of $\mathrm{SiO}_{2}$ square nanopillars 
Upon calculation of the thickness, shows that about $80 \mathrm{~nm}$ of gold stayed on the top of nanopillars matching the simulated model. As shown in the Figure 4.10.

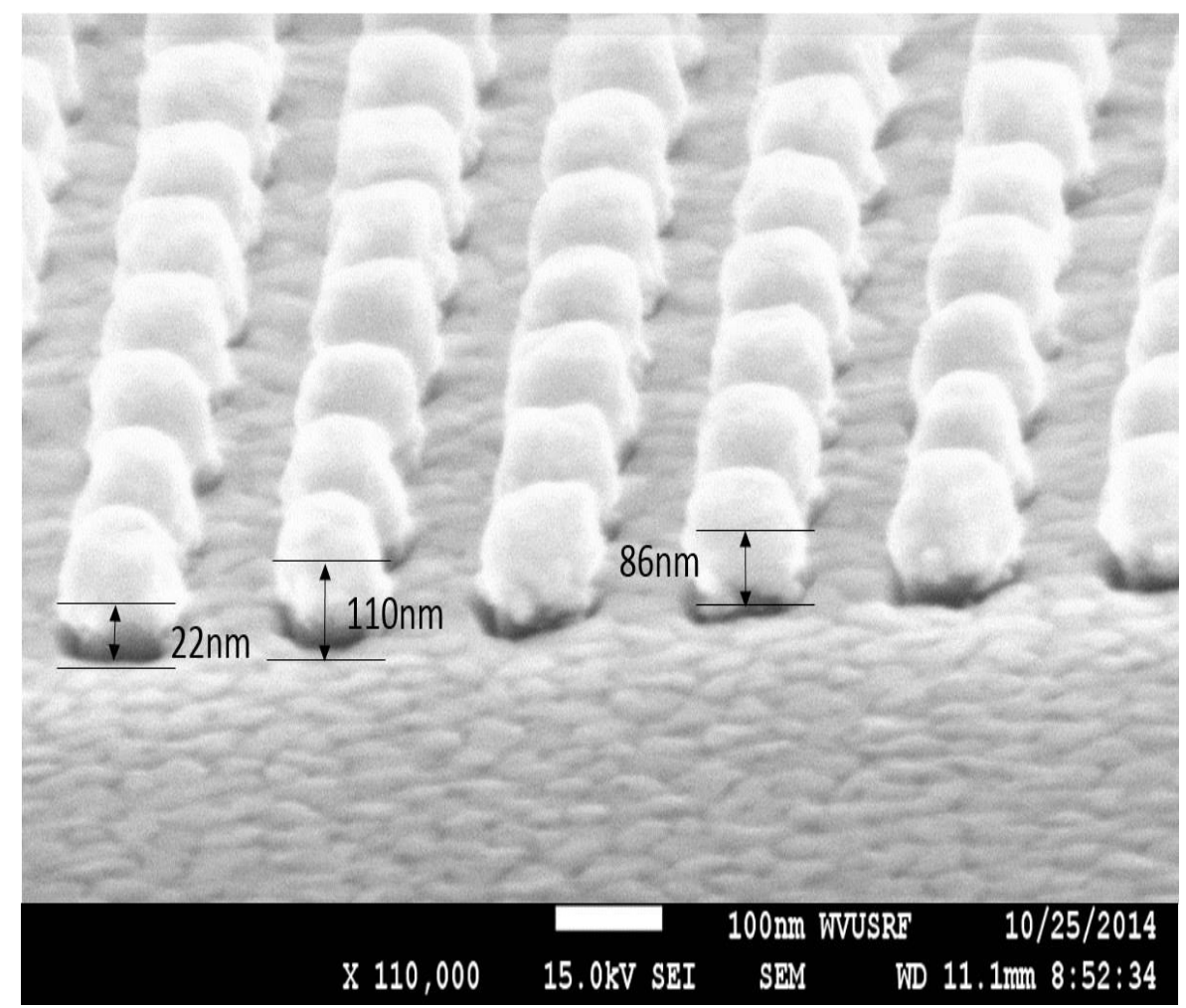

Figure 4-10:SEM image of fabricated structure at an angle of $70^{\circ}$

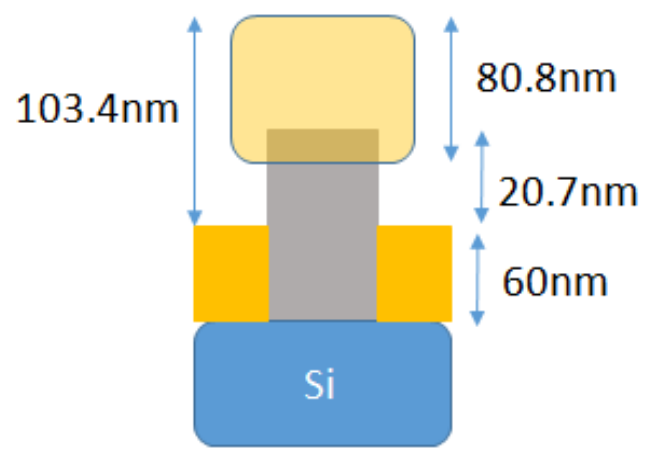

Figure 4-11: Optimized gold deposition on top of square nanopillars with deposition rate of $0.5 \mathrm{~A} / \mathrm{s}$ 


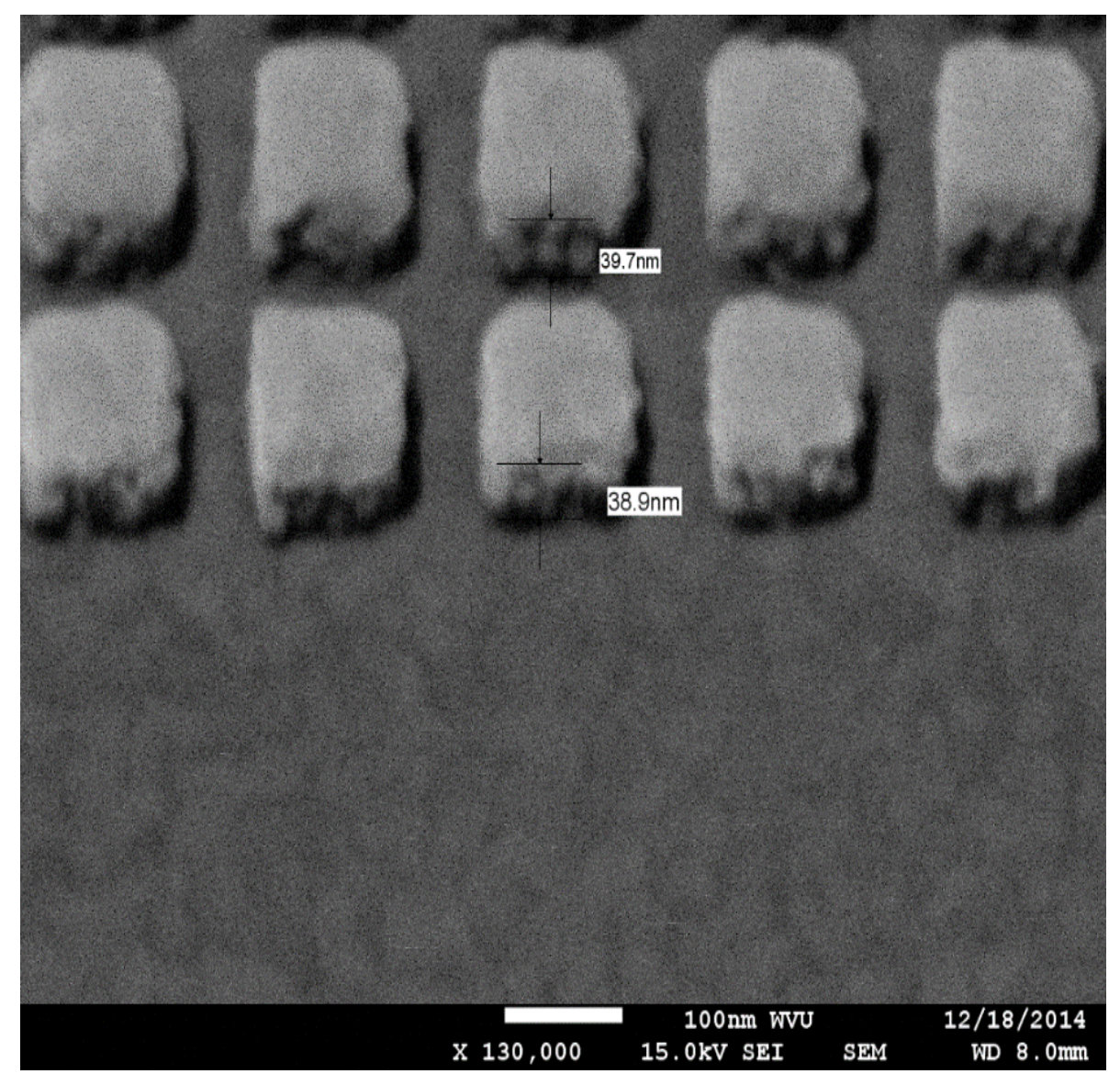

Figure 4-12:SEM image of fabricated structure at an angle of 30deg

\subsection{Spacer deposition}

The process of reduction in fluorescence is referred as quenching. Direct contact of the labels with the metal causes quenching of the dye. A thin layer of $\mathrm{SiO}_{2}$ is deposited on the gold nanopattern to avoid direct contact of the labels with the gold surface. To avoid quenching of quantum dots, $10 \mathrm{~nm}$ of $\mathrm{SiO}_{2}$ is deposited on top of gold using e-beam evaporator at the deposition rate of $0.2 \mathrm{~A} / \mathrm{s}$. The process is carried out in the vacuum of $8 \times 10^{-7}$ Torr. To deposit $\mathrm{SiO}_{2}$, the oxygen gas flow is maintained at $20 \mathrm{sccm}$ and to break the $\mathrm{O}_{2}$ molecules, the current of $1.31 \mathrm{~mA}$ and voltage of $165 \mathrm{~V}$ is applied. 


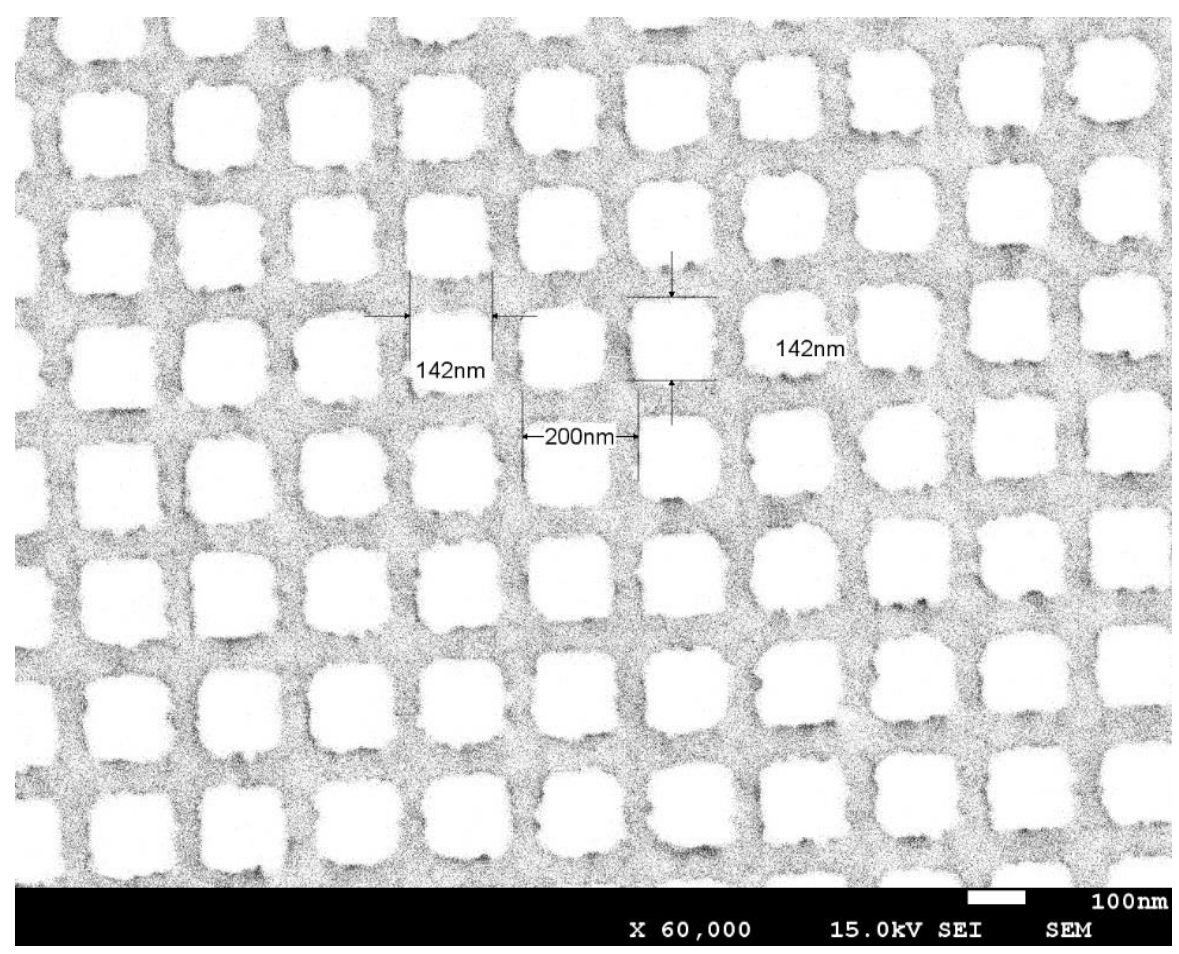

Figure 4-14:SEM picture showing square nanopillars after deposition of $\mathrm{SiO}_{2}$

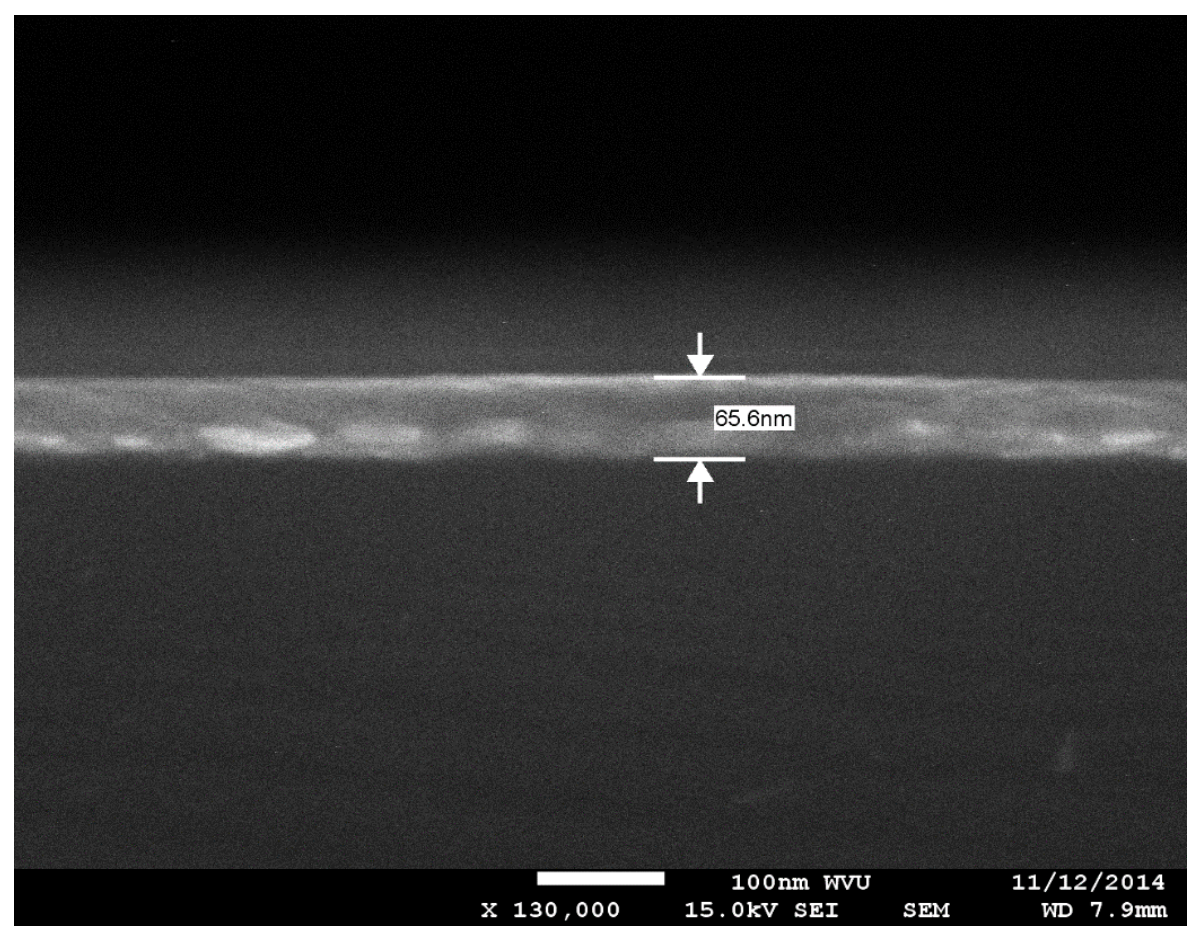

Figure 4-13:SEM image showing thickness of gold deposition at an angle of 90deg 


\subsection{Atomic Force Microscopy}

Atomic force microscopy is used to generate topographical images of the surface. It has capability of generating images with atomic resolution. AFM images are captured with Asylum MFP-3D Atomic Force Microscope (AFM). Images are processed using imaging software Gwyddion. Using tapping mode AFM, the deposition of 10nm dielectric layer is tested on the silicon substrate. The image showed the formation of $\mathrm{SiO}_{2}$ islands at the top of the sample.

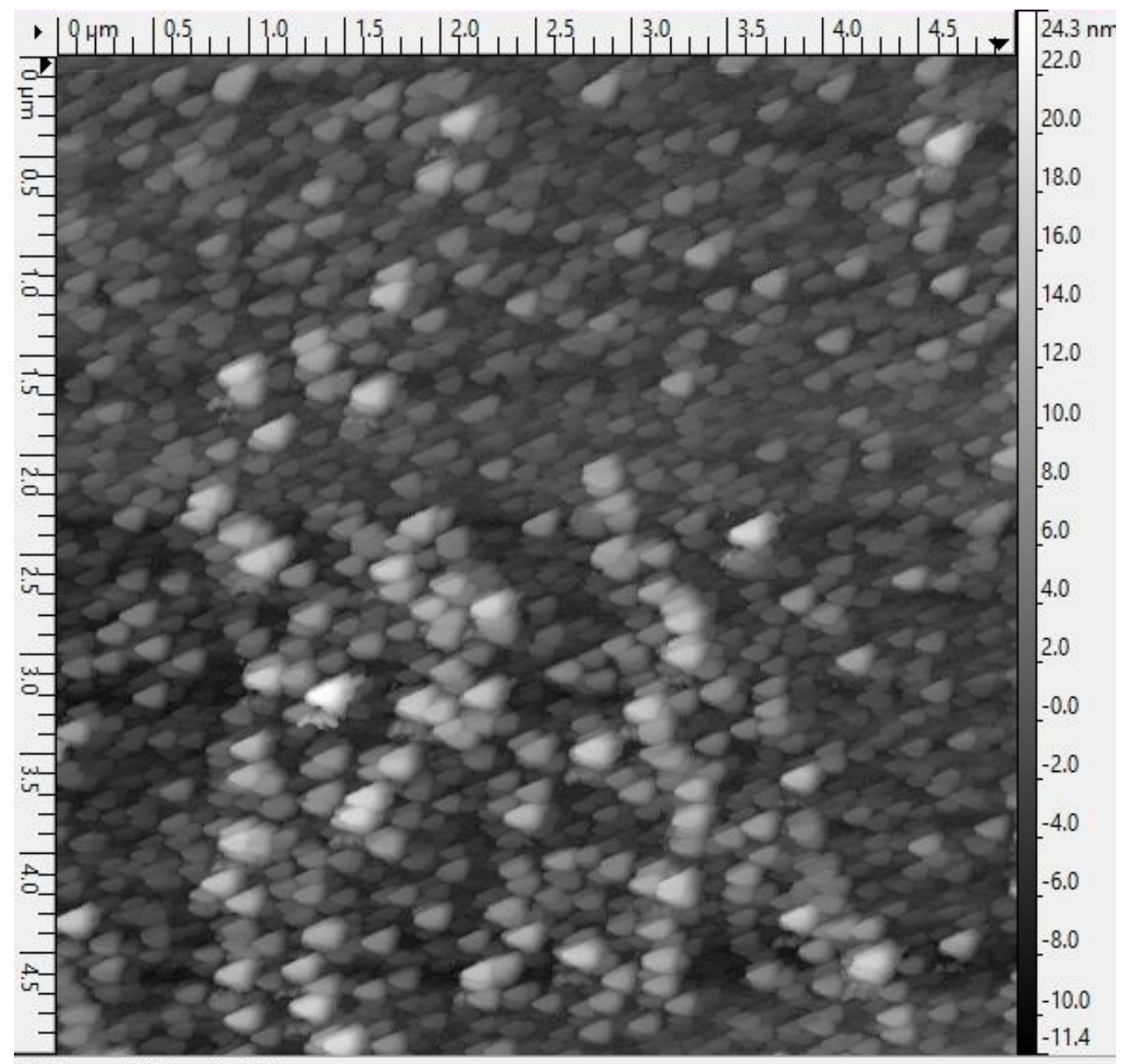

$(3.997 \mu \mathrm{m}, 0.034 \mu \mathrm{m}): 1.80 \mathrm{~nm}$

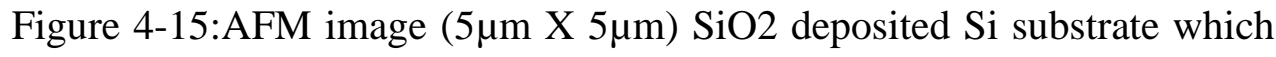
formed as islands 


\section{Chapter 5 \\ Characterization}

The electron beam lithography (EBL) fabricated nanostructures are characterized by scanning electron microscope (SEM) for the analysis of structure. The roughness of the deposition is characterized by using atomic force microscope (AFM). Optical microscope is used for the characterization of the structure and the fluorescence microscope is used to observe the fluorescence enhancement.

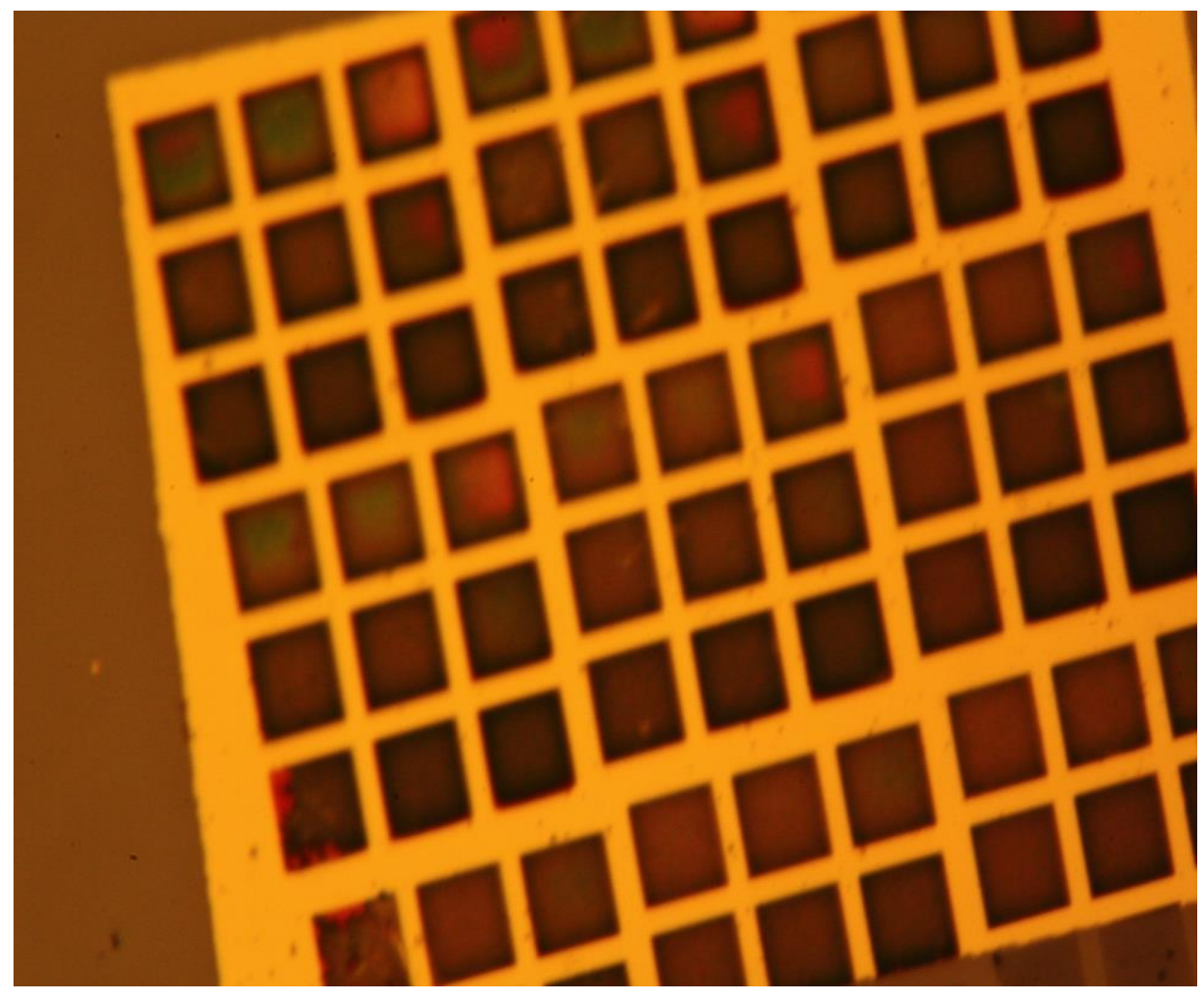

Figure 5-1: Optical image of the fabricated nanostructures with varying docaoes 


\subsection{Optical Characterization}

The visible region of the electromagnetic spectrum includes radiation at wavelengths between $400 \mathrm{~nm}$ to $700 \mathrm{~nm}$. The optical characterization is carried out using an olympus BH2 - UMA microscope located in class 100 cleanroom in WVU SRF, equipped with a white light source that is focused on the sample by a 50X microscope objective. Transmitted light is collected with 50X and NA=0.5 objective. The Figure 5.1 shows the optical image of the plasmonic nanopattern. 9 similar arrays of nanoarrays of 9 varying dosages are fabricated. As in the Figure 5.1, 9 larger squares represent 9 similar nanoarrays and the smaller squares within the larger squares represent the varying dosages.

Plasmonic nanostructures have designed to have less reflection. As shown in the Figure 5.1, the nanostructures are darker compared to the background.

\subsection{Fluorescence Spectroscopy}

Fluorescent spectroscopy is widely used in applications related to chemistry, photonics and material science, and molecular biology. Fluorescence is defined as the process of absorption of energy of light at one wavelength and nearly instantaneous re-emission at a longer wavelength. Surface enhanced fluorescence consists of modification of fluorescence intensities and lifetimes of the molecules on the metal surface [61]. The presence of metal nanostructures can reduce the lifetime of the molecule in the excited state and it results in quenching of fluorescence faster [62]. This is due to the metal particles resulting in strong electromagnetic (EM) response.

Fluorescence involves two processes, adsorption of photon, followed by emission. This process is similar to scattering. The enhanced local electric field leads to the change in absorption cross section, but the modification in the decay rates can be only modified by radiative enhancement.

Surface Enhanced Fluorescence is the enhancement of the fluorescence molecules in the vicinity of the nanoparticles. The presence of the nanoparticles reduces the lifetime of the molecule in the excited state. The theoretical explanation for fluorescence enhancement and the experimental results defines that the enhancement can occur only for fluorophore - metal enhancement can 
occur for distances greater than $10 \mathrm{~nm}$. The spacer layer leads to increase in relaxation rate and it helps to undergo more excitation and emission cycles before photo bleaching.

\subsubsection{Microscope Settings}

Ziess Violet Laser scanning confocal microscope is used to test the photoluminescence enhancement of the quantum dots.

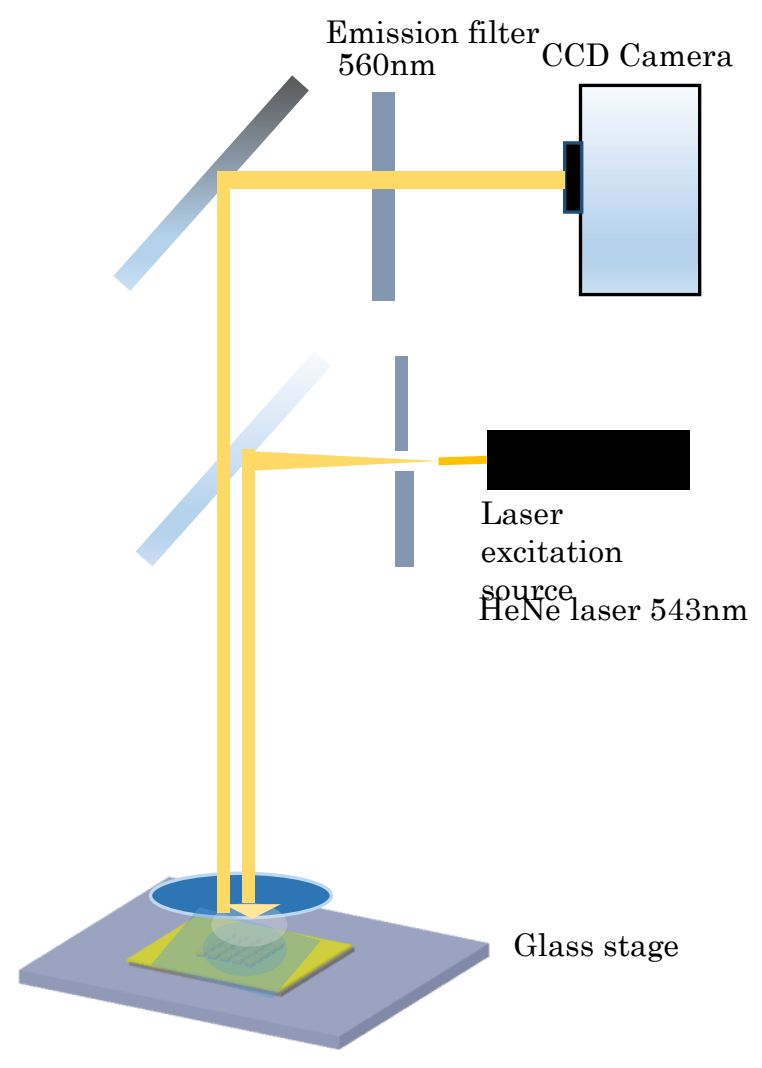

Figure 5-2: Block diagram of microscope 


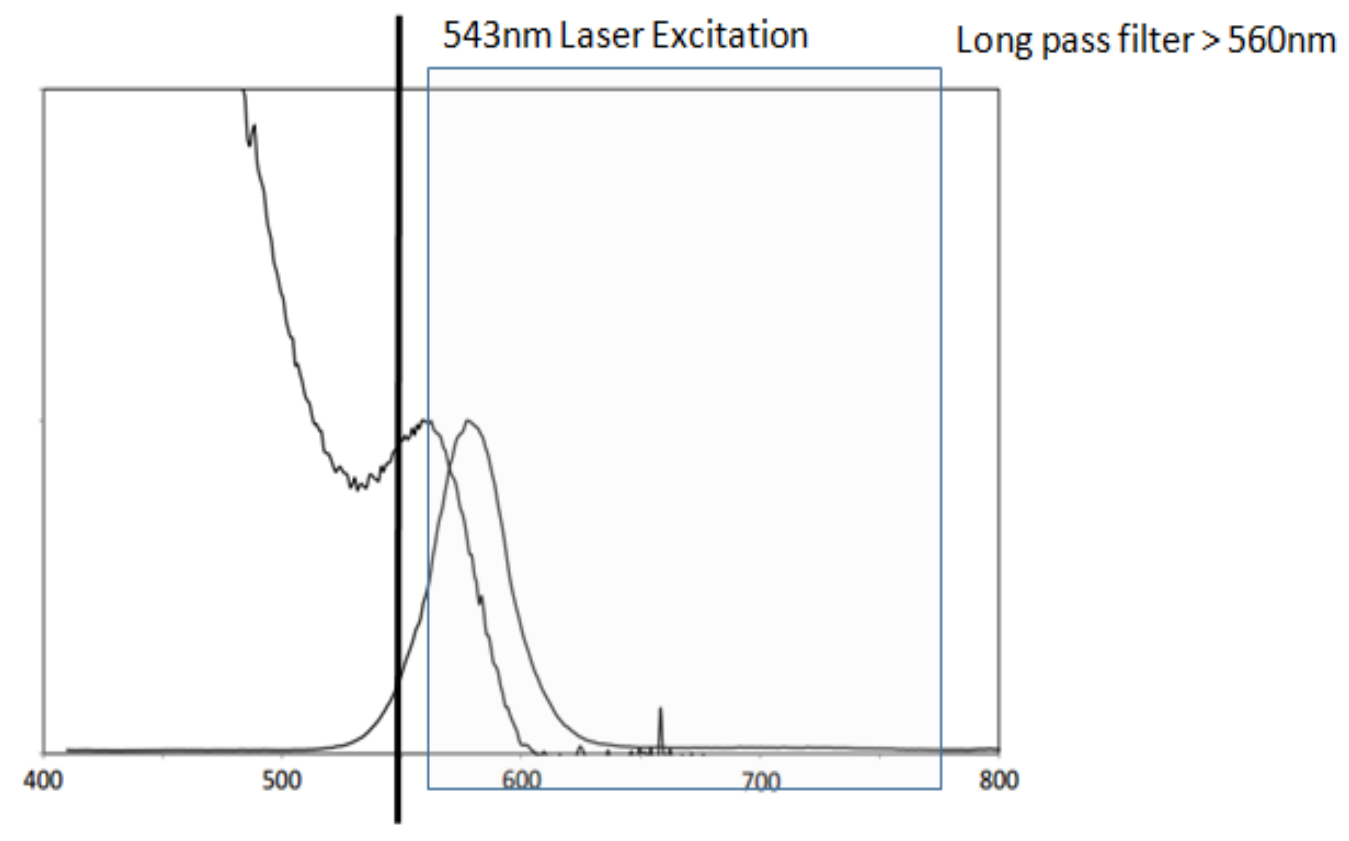

Figure 5-3: Spectrum of CdSe/ZnS quantum dots

$1.24 \times 10^{-6} \mathrm{M}$ Molar solution of $\mathrm{CdSe} / \mathrm{ZnS}$ quantum dots dispersed in toluene is drop coated on the plasmonic device and the fluorescence images are collected by Zeiss confocal microscope using $\mathrm{HeNe} 543 \mathrm{~nm}$ laser with power of 1.2 watts is used for the excitation. Only $4 \%$ power of the total power is used for excitation with excitation wavelength $543 \mathrm{~nm}$. The emission filter configuration is set to $560 \mathrm{~nm}$ with a longpass filter, which allows only the wavelengths above $560 \mathrm{~nm}$. The plain apochromat oil immersion objective (63X, 1.40 NA) is used. The scanned size of the image is 512 x 512 and the emission of the dye is collected using a long pass filter of wavelength $560 \mathrm{~nm}$. The emission from the dye is collected in the range of $560 \mathrm{~nm}-700 \mathrm{~nm}$. The images were obtained before and after adding the quantum dot solution on the top of sample.

\subsubsection{Optical filters}

Optical filters are chosen to be optimal and to pass selectively pass a portion of visible spectrum. The light source in combination with excitation filter allows only light of particular wavelengths and the emission filter allows the fluorescence from the samples to pass to the decoder and blocks the stray light form the interfering components in the sample and light source. The filters are used to select the desired spectral band. The images are processed using Zen Lite software.

Long pass filters pass a broad band of light above a particular wavelength. For example, a 560nm filter with long pass filter, will pass light from 560nm to 700nm. 


\subsubsection{Reflection Images}

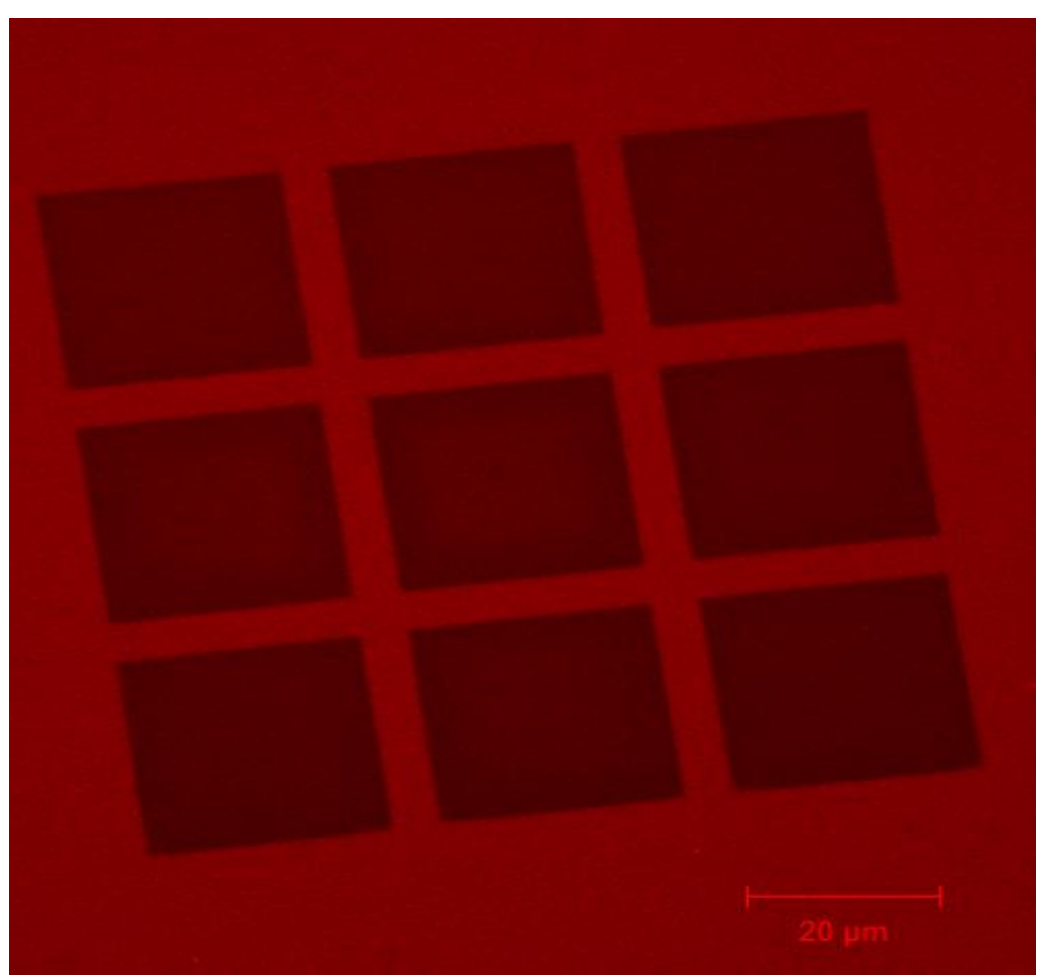

Figure 5-4: Reflection image of the sample focused with a laser

The Nanostructure is modeled to have high scattering. The total excitation energy can be converted as the sum of transmission, reflection, scattering and absorption. As the silicon substrate is a solid sample, the transmission into the sample is zero.

The incident light till a particular frequency can oscillate the free electrons on the top of the metal surface and above the particular frequency, the light is absorbed into the material as is converted into heat. The operating wavelength of the incident light is above the plasma frequency, hence the light is all scattered rather than being absorbed. Scattered light will cause increase in electric field around the nanostructure which increases the quantum yield if the fluorophore in its vicinity. 
Reflection + Scattering + Absorbtion $=$ Incident field

As seen from the picture, the nanostructure shows low reflection compared to the plain gold, so the amount of extinction (scattering + absorption) is higher on the nanopattern compared with the plain gold surface. The scattered light can be visualized by the normally incident dark field microscopy technique, but is not available in WVU SRF.

As scattered light cannot be captured by the optical microscope, the nanopillars are dark compared to the background as in the Figure 5.4.

\subsubsection{Fluorescent Microscope Images}

The scattered light increases the electric field around the nanostructure and the quantum yield of the fluorophores in the vicinity of the nanostructures increases due to enhancement in electric field. As shown in the Figure 5.5 the nanostructures are modeled to have high scattering field intensity compared to the background plain gold material. The enhancement in fluorescence is only observed within $200 \mathrm{~nm}$ near to the nanostructure as the near field enhancement can be

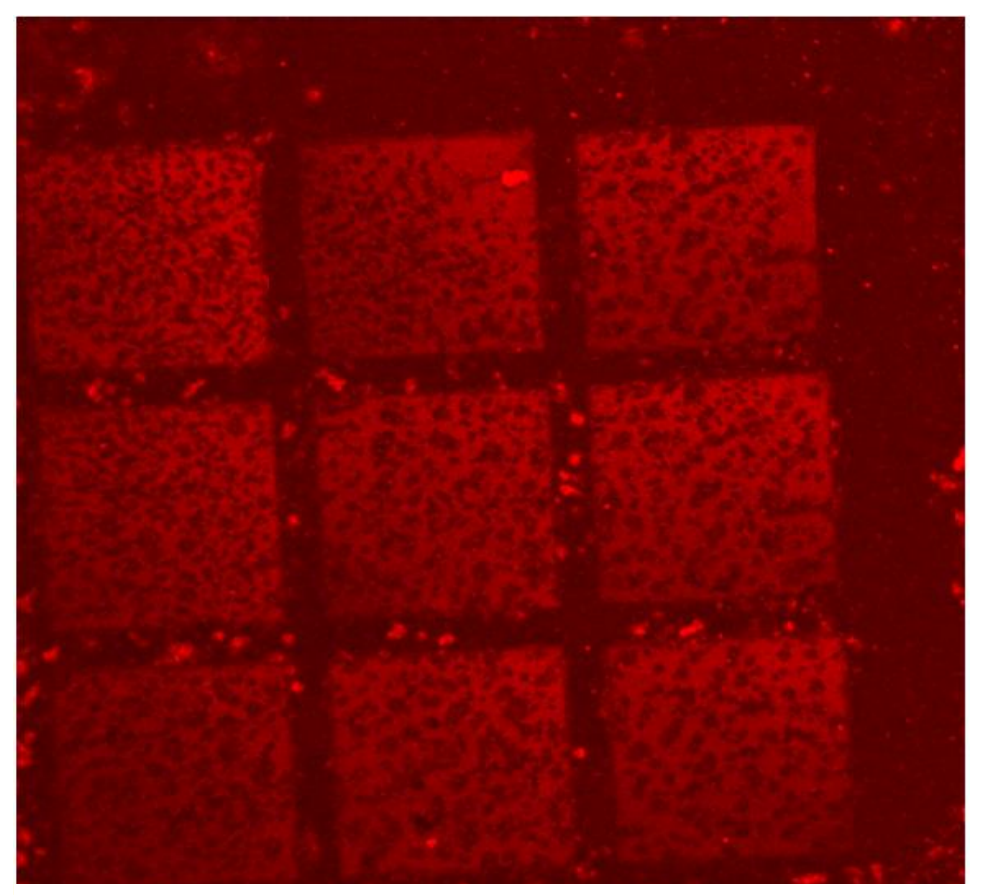

Figure 5-5: Fluorescent microscope excited with a laser source 
observed only in the vicinity of the nanostructures.

To test the nanostructures, $\mathrm{CdSe} / \mathrm{ZnS}$ quantum dots dispersed in toluene are used. Toluene has higher evaporation rate. As toluene evaporates settling down the quantum dots on the substrate helped to get quantum dots in the vicinity of the nanostructure

\subsection{Results analysis}

Data represented in the following tables is the pixel values of the $R, G$, and $B$ on the nanopattern and in the background.

Table 5-1:Comparing pixel values on (left) plasmonic nanostructure and (right) thin gold film

\begin{tabular}{|l|l|l|}
\hline \multicolumn{1}{|r|}{$\mathbf{R}$} & G & B \\
\hline 174 & 0 & 0 \\
\hline 167 & 0 & 0 \\
\hline 162 & 0 & 0 \\
\hline 503 & 0 & 0 \\
\hline 167.7 & 0 & 0 \\
\hline
\end{tabular}

\begin{tabular}{|l|l|l|l|}
\hline \multicolumn{1}{c|}{} & $\mathbf{R}$ & $\mathbf{G}$ & $\mathbf{B}$ \\
\hline 81 & 0 & 0 \\
\hline \multirow{5}{*}{ Total } & 250 & 0 & 0 \\
\cline { 2 - 4 } & 82 & 0 & 0 \\
\cline { 2 - 4 } Average & 83.3 & 0 & 0 \\
\cline { 2 - 4 } & & 0 & 0 \\
\hline
\end{tabular}

Enhancement: $\frac{173.4}{84}=2.01$ times compared to the background,

2.01 times the enhancement is achieved on the nanopattern compared to the plain gold surface.

MATLAB code is written to observe the enhancement of the fluorescence. It is observed that the enhancement of the fluorescence compared to the background is about 2.07 times enhancement. MATLAB program is given in the appendix. 
Metal enhanced fluorescence theory suggests that the enhancement is due to the increase in intensity of the excitation field which is localized onto the metallic nanostructures and due to the increase in radioactive decay rate of the fluorophore which can be interpreted by the lifetime measurements for the fluorophores to support this interpretation.

The efficiency of the metal enhanced fluorescence depends on the distance between the metallic nanostructure and the quantum dots and the fluorescence emission from $0-10 \mathrm{~nm}$ is mostly quenched by metal nanoparticles.

Electron beam lithography is used to fabricate the nanostructures through which the size and shape and distance between the nanostructures can be effectively and accurately designed. 


\section{Chapter 6 Conclusion and Future scope}

The sensitive DNA detection is based on the enhancement of the labelled dye attached to DNA with localized surface plasmon resonance (LSPR) sensor. The enhancement results in a significant decrease of the limit of detection labeled DNA. Applications of localized surface plasmon resonance (LSPR) such as surface enhanced fluorescence (SEF) devices are growing. The localized surface plasmon resonance in gold nanopillars is used in this thesis as the basis of a labelled biosensor detection scheme. This is accomplished by enhancement of the luminescence of the quantum dots due to high electric fields generated at plasmonic frequency by gold nanopillar arrays. Plasmonic biosensor arrays for labeled and high throughput detection of DNA were simulated, fabricated and tested.

Metallic nanostructures supporting localized surface plasmons (LSPs) which can amplify the signal in fluorescence biosensors. Finite difference time domain (FDTD) software, Lumerical is used for simulations, a novel LSP structure is designed that exhibit resonance overlapping with absorption and emission wavelengths of assumed fluorophore. The shape and size of the metal and distance between the nanostructures play a typical role in the enhancement of the electric field around the nanostructures.

Understanding and investigating of the parameters that affect the localized surface plasmon resonance spectrum is important for design and fabrication of LSPR devices. The extinction spectra of Au nanopillars on silicon substrate is simulated by finite difference time domain (FDTD) method, using FDTD software from Lumerical simulation. This thesis studies different parameters, including material effect, geometrical structures, such as the spacing between gold 
nanostructures which affect the LSPR spectrum properties such as plasmon wavelength which in turn affects the enhancement factor. The dependence of the LSPR wavelength is investigated and plasmonic peak sensitivity on the diameter and height of nanopillars and gold deposition thickness, with the aim to find an optimum configuration for effective LSPR based biological sensing. 3D finite difference time domain simulations confirm that high local electric fields in between the nanopillars which results in the enhancement of luminescence of quantum dots.

Dense $\mathrm{SiO} 2$ nanoparticle arrays on silicon substrate are fabricated by electron beam lithography (EBL) and the patterned structure is subsequently covered with thin layers of Au. The sensor array was composed of a $60 \mathrm{~nm}$ thick gold deposited on $115 \mathrm{~nm}$ diameter, 200nm gap $\mathrm{SiO}_{2}$ nanopillars array fabricated on silicon substrate. Each array size was $20 \mu \mathrm{m}$ X $20 \mu \mathrm{m}$.

A Normally focused light wave in these metallic nanostructures generated resonant surface plasmons at a wavelength of about 560nm in a toluene environment. Luminescence enhancement of quantum dots on plasmonic arrays was observed by testing the enhancement of cadmium selenide zinc sulfide $(\mathrm{CdSe} / \mathrm{ZnS}$ ) quantum dots (from Nanomaterials and Nanofabrication (N \& N) Laboratories) with excitation peak wavelength at $560 \mathrm{~nm}$ and emission peak wavelength at $585 \mathrm{~nm}$. Using the resonant wavelength in the nanopillar array, we achieved detection sensitivity up to 2.01 times enhancement.

This thesis is comprised of simulated and experimental results, combined with high resolution scanning electron microscopy images.

\subsection{Future scope}

For biosensor applications, it's desirable that the plasmonic nanostructures to be integrated into microfluidic channels. Plasmonic nanostructures can be integrated into a chip based biosensor array for label based detection to create a portable sensitive biosensor.

The fluorescence enhancement due is to the light scattered by the nanostructures. The scattering peak of nanostructures is taken from simulation design, rather than that we can use UV-Vis absorption spectra to find the plasmonic peak. Near field scanning optical microscope (NSOM) 
can be used to characterize the plasmonic nanostructures to find the electric field enhancement at the vicinity of the nanostrutcures.

Deposition of the spacer layer using atomic layer deposition can increase the efficiency of the deposition and further the fluorescence enhancement for varying spacer layer deposition thickness can be accurately calculated.

By preparing solutions with varying molar concentrations can be useful in calculating the best concentration suitable for quantum dots dispersed in toluene to generate maximum enhancement. Agglomeration of quantum dots due to the settling of the quantum dots on the sample. In order to avoid it, a flow cell configuration can be built to allow continuous flow of quantum dots above the sample, where the flow avoids the agglomeration of the quantum dots. 


\section{$\underline{\text { References }}$}

[1] A. H. F. a. H. M. Free, "Self testing, An emerging component in clinical chemistry," CLIN. CHEM., vol. 30, no. 6, pp. 829-838, 1984.

[2] M. F. Burritt, "Current Analytical Approaches to Measuring Blood Analytes," CLIN. CHEM, vol. 36, no. 8, pp. 1562-1566, 1990.

[3] P. B. Luppa, L. J. Sokoll and D. W. Chan, "Immunosensors-principles and applications to clinical chemistry," Clinica Chimica Acta, vol. 314, no. 1-2, p. 1-26, 2001.

[4] T. Tokuda, M. Takahashi, K. Uejima, K. Masuda, T. Kawamura, Y. Ohta, M. Motoyama, T. Noda, K. Sasagawa, T. Okitsu, S. Takeuchi and J. Ohta, "CMOS image sensor-based implantable glucose sensor using glucose-responsive fluorescent hydrogel," Biomedical optics express, vol. 5, no. 11, p. 3859-3870, 2014.

[5] "NANOSAFE," West Virginia Univeristy, [Online]. Available: http://nanosafe.wvu.edu/.

[6] M. S. A. K. J. D. a. L. H. B. Hamza, "Photonic Crystal Bioensors," in Biosensors Based on Nanomaterials and Nanodevices, Taylor \& Francis, August 2014. 
[7] A. K. C. K. Y. L. a. J. D. B. Hamza, "Fluorescence Enhancement in a Polymer-based Photonic Crystal Biosensor," SPIE, vol. 7888, p. 788804, 2011.

[8] K. G. I. M. J. M. E. L. J. a. G. C. Aslan, "Metalenhanced fluorescence: an emerging tool in biotechnology," Current Opinion in Biotechnology, vol. 16, pp. 55-62, 2005.

[9] K. A. Chris D. Geddes, Metal Enhanced Fluorescence, wiley.

[10] D. R. H. C. F. Bohren, Absorption and Scattering of Light by Small particles, New York: Wiley, 1983.

[11] F.-G. a. I. e. Banica, Chemical Sensors and Biosensors: Fundamentals and Applications., Chichester, West Sussex, United Kingdom: Wiley, 2012.

[12] O. W. R. Narayanaswamy, "Optical Sensors," Springer, Newyork, 2004.

[13] S.Zeng, D. Baillargeat, H.-P. Ho and K.-T. e. a. Yong, "Nanomaterials enhanced surface plasmon resonance for biological and chemical sensing applications," Chemical Society Reviews, vol. 43, no. 10, p. 3426-3452, 2014.

[14] G. A. Urban, "Micro- and nanobiosensors—-state of the art and trends," Meas. Sci. Technol, vol. 20, p. 012001, 2009.

[15] M. Marazuela and M. Moreno-Bondi, "Fiber-optic biosensors - an overview," Analytical and Bioanalytical Chemistry, vol. 372, no. 5-6, p. 664-682, 2002. 
[16] J. Pickup, Z. Zhi, F. Khan, T. Saxl and D. Birch, "Nanomedicine and its potential in diabetes research and practice," Diabetes Metab Res Rev, vol. 24 , no. 8, p. 604-10, 2008.

[17] F. S. L. a. C. R. Taitt, Optical Biosensors (Second Edition), Elsevier, 2008.

[18] M. Velasco-Garcia, "Optical biosensors for probing at the cellular level: A review of recent progress and future prospects," Seminars in Cell \& Developmental Biology, vol. 20, p. 2733, 2009.

[19] K. A. T. M. Hoa XD, "Towards integrated and sensitive surface plasmon resonance biosensors: a review of recent progress.," Biosens Bioelectron, vol. 23, no. 2, pp. 151-60., 2007.

[20] J. X. W. Z. a. H. C. X. Luo, "Glucose biosensor based on ENFET doped with $\mathrm{SiO} 2$ nanoparticles," Sensors and Actuators, vol. 97, no. no. 2-3, p. 249-255, 2004.

[21] J. M. W. S. C. M. a. N. J.-R. A. P. Soldatkin, "A novel urea sensitive biosensor with extended dynamic range based on recombinant urease and ISFETs," Biosensors and Bioelectronics, vol. 19, no. 2, p. 131-135, 2003.

[22] e. b. T. Vo-Dinh, Biomedical Photonics Handbook, Second Edition: Biomedical Diagnostics, CRC press, Taylor and Fransis Group.

[23] A. Touhami, "Biosensors and Nanobiosensors: Design and Applications," Nanomedicine, pp. 374-403. 
[24] R. M. Harsh Sharma, "Review of biosensors for foodborne pathogens and toxins," Sensors and Actuators, vol. 183, p. 535-549, 2013.

[25] S. A. H. T. S. a. K. T. V. G. T. Hien Nguyen, "Fluorescence-Based Optical Fiber Sensor for Cocaine Using a Molecularly Imprinted Polymer as the Recognition Element.," IEEE SENSORS JOURNAL, vol. 12, no. 1, pp. 255-260, 2012.

[26] H. C. S. a. P. F. Ouyang, "Quantitative analysis of the sensitivity of porous silicon optical biosensors," Applied Physics Letters, vol. 88, no. 16, 2006.

[27] D. e. a. Kim, " Simultaneous measurement of refractive index and temperature based on a reflection-mode long-period grating and an intrinsic Fabry-Perot interferometer sensor.," Opt. Lett.,, vol. 30, no. 22, 2005.

[28] G. B. M. V. D. d. C. ,. V. P. ,. M. S. ,. G. B. ,. M. Grande, "2D plasmonic gold nano-patches for linear and nonlinear applications," ELSEIVER, pp. 234-237, 17 April 2013.

[29] M. e. a. Levene, " Zero-Mode Waveguides for Single-Molecule Analysis at High Concentrations," Science, vol. 299, no. 5607, 2003.

[30] W. Moerner, "New directions in single-molecule imaging and analysis," Proceedings of the National Academy of Sciences of the United States of America, vol. 104, no. 31, 07/2007.

[31] V. L. S. W.G. Cox, "Fluorescent DNA hybridization probe preparation using amine modification and reactive dye coupling.," Biotechniques, vol. 36, p. 114, 2004. 
[32] S. M. R. G. L. T. P. C. D. S. Strianese M1, "Fluorescence-based biosensors.," Methods Mol Biol., pp. 193-216, 2012.

[33] B. (. B. V. Lotsch and G. (. G. A. Ozin, "Photonic Clays: A New Family of Functional 1D Photonic Crystals," ACS NANO, vol. 2, no. 10, pp. 2065-2074, OCT 2008.

[34] Z. (. Z. 1. ]. By:Wang, J. (. J. 1. ]. Zhang, J. (. J. 1. ]. Xie, Y. (. Y. 1. ]. Yin, Z. (. Z. 1. ]. Wang, H. (. H. 1. ]. Shen, Y. (. Y. 1. ]. Li, J. (. J. 1. ]. Li and S. (. Cui, "Patterning Organic/Inorganic Hybrid Bragg Stacks by Integrating One-Dimensional Photonic Crystals and Macrocavities through Photolithography: Toward Tunable Colorful Patterns as Highly Selective Sensors," ACS APPLIED MATERIALS \& INTERFACES, vol. 4, no. 3, pp. 13971403, MAR 2012.

[35] J. (. J.-T. 1. ]. Zhang, L. (. L. 1. ]. Wang, D. (. D. N. 1. ]. Lamont, S. (. S. S. 2. ]. Velankar and S. (. S. A. 1. ]. Asher, "Fabrication of Large-Area Two-Dimensional Colloidal Crystals," ANGEWANDTE CHEMIE-INTERNATIONAL EDITION, vol. 51, no. 5, pp. 61176120, 2012.

[36] P. Y. Y. Dr. Jianping Ge, "Responsive Photonic Crystals," Angew. Chem. Int. Ed. , vol. 50, p. $1492-1522,20$ January 2011.

[37] Y. (. Y. Xia, B. (. B. Gates, Y. (. Y. Yin and Y. (. Y. Lu, "Monodispersed colloidal spheres: Old materials with new applications," ADVANCED MATERIALS, vol. 12, no. 10, pp. 693713, MAY 172000.

[38] H. (. H. 1. ]. Wang and K. (. K.-Q. 1. ]. Zhang, "Photonic Crystal Structures with Tunable Structure Color as Colorimetric Sensors," SENSORS, vol. 13, no. 4, pp. 4192-4213, APR 
2013.

[39] S. M. S. a. B. T. Cunningham, "Label-free cell-based assays using photonic crystal optical biosensors," Analyst, vol. 136, pp. 1090-1102, 2011.

[40] M. S. A. K. J. D. a. L. H. B. Hamza, "Photonic Crystal Bioensors," in iosensors Based on Nanomaterials and Nanodevices, Taylor \& Francis, 2014, p. Chapter 8.

[41] P. A. Van Engelenburg SB1, "Quantification of real-time Salmonella effector type III secretion kinetics reveals differential secretion rates for SopE2 and SptP.," Chem Biol., vol. 15, no. 6, pp. 619-28, 2008.

[42] A. K. L. H. Y. L. R. C. a. J. D. B. Hamza, "Replica Molding of Nano-scale TwoDimensional Photonic Crystals in Poly(dimethylsiloxane) and Epoxy for Light Manipulation in the Visible Range," Microelectronic Engineering.

[43] A. K. L. H. Y. L. a. J. D. B. Hamza, "Direct Fabrication of Two-dimensional Photonic Crystal Structures in Silicon Using Positive and Negative Hydrogen Silsesquioxane (HSQ) Patterns," Microelectronic Engineering, vol. 91, p. 70, 2012.

[44] A. K. L. A. H. R. L. C. Y. L. a. J. D. B. M. Hamza, "The Modeling, Fabrication, and Optical Characterization of Silicon and Polymer-based Photonic Crystals for Biosensing Applications," ECS Trans. , vol. 41, no. 38, p. 1, 2012.

[45] A. K. C. K. Y. L. a. J. D. B. Hamza, "Fluorescence Enhancement in a Polymer-based Photonic Crystal Biosensor," Proc. SPIE, no. 7888, p. 788804, 2011. 
[46] K. I. edited by James W. M. Chon, Nanoplasmonics: Advanced Device Applications, CRC press, Taylor and Fransis group, 2014.

[47] L. Z. J. Z. a. H. W. Wanbo Li, "Well-designed metal nanostructured arrays for label-free plasmonic biosensing," J. Mater. Chem. C, vol. 3, pp. 6479-6492, 2015.

[48] I. G. C. D. G. J. R. L. J. Malicka, "Metal-enhanced emission from indocyanine green: a new approach to in vivo imaging," J. Biomed.Opt., , vol. 8, p. 472-478, Jul 2003.

[49] G. C. G. I. M. J. G. Z. A. K. L. J. M. E. Z. J. B. R. H. J. Lakowicz J.R., "Advances in surface-enhanced fluoresence," J Fluoresc, vol. 14, no. 4, pp. 425 -41, 2004.

[50] J. R. Lakowicz, J. Malicka, S. D'Auria and I. Gryczynski, "Release of the self-quenching of fluorescence near silver metallic surfaces," Analytical Biochemistry, vol. 320, no. 1, p. 1320, Sep 2002.

[51] B. R. B. E. U. S. H. R. W. S. K. A. Becker, "Macromolecular contrast agets for optical imaging of tumors: Comparision of indotricarbocyanine-labeled human serum albumin and trasferring," Photochem. Photobiol., vol. 72, pp. 234-241, Jun 2000.

[52] J. F. J. K. F. G. H. F. Schutt, "Indocyanine green angiography in the presence of subretinal or intraretinal haemorrhages: Clinical and experimental investigations," Clin. Exp. Ophthalmol., vol. 30, pp. 110-114, Feb 2002.

[53] A. G. Y. M. S. B. C. V. Ntziachristos, "Concurrent MRI and diffuse optical tomography of breast after indocyanine green enhancement," Proc. Natl. Acad. Sci., vol. 97, p. 2767-2772, Mar. 2000.. 
[54] I. G. J. R. L. J. Malicka, "DNA hybridization assays using metal-enhanced fluorescence," Biochem. Biophys. Res. Commun., vol. 306, p. 213-218, Apr. 2003.

[55] L. M. A. D. T. F. D. S. L. M. M. D. V. F. C. R. C. A. R. R. P. P. POMPA*, "Metalenhanced fluorescence of colloidal nanocrystals with nanoscale control," Nature, vol. 1, 2014.

[56] M. Kerker, "The optics of colloidal silver: Something old and something new," Journal of Colloid and Interface Science, vol. 105, no. 2, p. 297-314, 1985.

[57] M. Faraday, "The Bakerian Lecture: Experimental Relations of Gold (and Other Metals) to Light," Philosophical Transactions of the Royal Society of London, vol. 147, pp. 145-181, 1857.

[58] J. H. F. Eliza Hutter, "Exploitation of localized surface plasmon resonance," Adv. Mater., vol. 16, p. 1685-1706, Oct. 2004.

[59] S. Underwood and P. Mulvaney, "Effect of the Solution Refractive Index on the Color of Gold Colloids," Langmuir, vol. 10, no. 10, p. 3427-3430, 1994.

[60] P. Mulvaney, "Surface Plasmon Spectroscopy of Nanosized Metal Particles," Langmuir, vol. 12, no. 3, p. 788-800, 1996.

[61] D. A. M. C. Daniel, "Gold nanoparticles: Assembly, supramolecular chemistry, quantumsize-related properties, and applications toward biology, catalysis, and nanotechnology," Chem. Rev. , vol. 104, p. 293-346, Jan. 2004. 
[62] O. Stranik, H. M. McEvoy, C. McDonagh and B. D. MacCraith, "Plasmonic enhancement of fluorescence for sensor applications," Sensors and Actuators B: Chemical, vol. 107, no. 1, p. 148-153, 2005.

[63] J.-J. Greffet, Introduction to Surface Plasmon Theory, vol. 167, Springer Berlin Heidelberg, 2012, p. 105-148.

[64] G. Mie, "Beiträge zur Optik trüber Medien, speziell kolloidaler Metallösungen," Ann. Phys. (Annalen der Physik), vol. 330, no. 3, p. 377-445, 1908.

[65] S. Fayyaz, "Enhancing Raman and Fluorescence Spectroscopies with Nanosphere Lithography Platforms," University of Western Ontario - Electronic Thesis and Dissertation Repository.

[66] D. Brouard, M. L. Viger, A. G. Bracamonte and D. Boudreau, "Label-Free Biosensing Based on Multilayer Fluorescent Nanocomposites and a Cationic Polymeric Transducer," ACS Nano, vol. 5, no. 3, pp. 1888-1896, 2011.

[67] C. D. G. a. J. Lakowicz, Metal-enhanced fluorescence, J. Fluoresc., 2002, pp. 121-129.

[68] Y. Xu, G. Lei, A. C. Booker, K. A. Linares, D. L. Fleming, K. Meehan, G.-Q. Lu, N. G. Love and B. J. Love, "Maximizing dye fluorescence via incorporation of metallic nanoparticles in solution," Lab-on-a-Chip: Platforms, Devices, and Applications, Proceedings of SPIE, vol. 5591, pp. 174-184, 2004.

[69] A. N. J. Gersten, "Spectroscopic properties of molecules interacting with small dielectric particles," J. Chem. Phys., vol. 75, pp. 1139-1152, Aug 1981. 
[70] G. L. A. C. B. K. A. L. D. F. K. M. G. Q. L. N. G. L. B. J. L. Y. Xu, "Maximizing dye fluorescence via incorporation of metallic nanoparticles in solution," Lab-on-a-Chip: Platforms, Devices, and Applications, Proceedings of SPIE., vol. 5591, p. 174-184, 2004.

[71] J. R. Lakowicz, "Radiative decay engineering: Biophysical and biomedical applications," Anal. Biochem, vol. 298, pp. 1-24, Oct 2001.

[72] M. Muller, Introduction to confocal fluorescence microscopy, Bellingham, Wash.: SPIE, 2006.

[73] M. Moskovits, "Surface-enhanced Raman spectroscopy: a brief retrospective," Journal of Raman Spectroscopy, vol. 36, no. 6-7, p. 485-496, 2005.

[74] Ito and Okazaki, "Pushing the limits of lithography," Nature (Nature), vol. 406, no. 6799, p. 1027-1031, 2000.

[75] H. Duan, A. I. Fernández-Domínguez, M. Bosman, S. A. Maier and J. K. W. Yang, "Nanoplasmonics: classical down to the nanometer scale," Nano Lett. (Nano letters), vol. 12, no. 3, p. 1683-1689, 2012.

[76] B. Zhang, W. H. P. R. Lee, I. W. Y. Kholmanov, H. Li, H. Ji and R. S. Ruoff, ACS Nano, vol. 6, pp. 2471-2476, 2012.

[77] G. P.-B. M. S. P. C. B. V. L. P. A. F. Komurian-Pradel, "Quantitation of HCV RNA using real-time PCR and fluorimetry," J. Virol. Methods,, vol. 95, pp. 111-119, Mar 2001. 
[78] "Lumerical simulation," [Online]. Available: http://www.lumerical.com/.

[79] "http://www.onetouch.com/onetouch-ultra2," One touch. [Online]. 


\section{APPENDIX A: PREPROCESS PREPARATION}

\section{E - BEAM LITHOGRAPHY CAD FILE}

To fabricate the square nanopillars by e beam lithography, SEM equipped with nanopattern generating system is used. The nanopattern generating system utilizes a software, Design CAD express to create a design CAD file, i.e, creating the nanostructures in a CAD based environment. Run file actually helps in creating the e-beam dosages and then integrating the design $\mathrm{CAD}$ file to the e-beam writing.

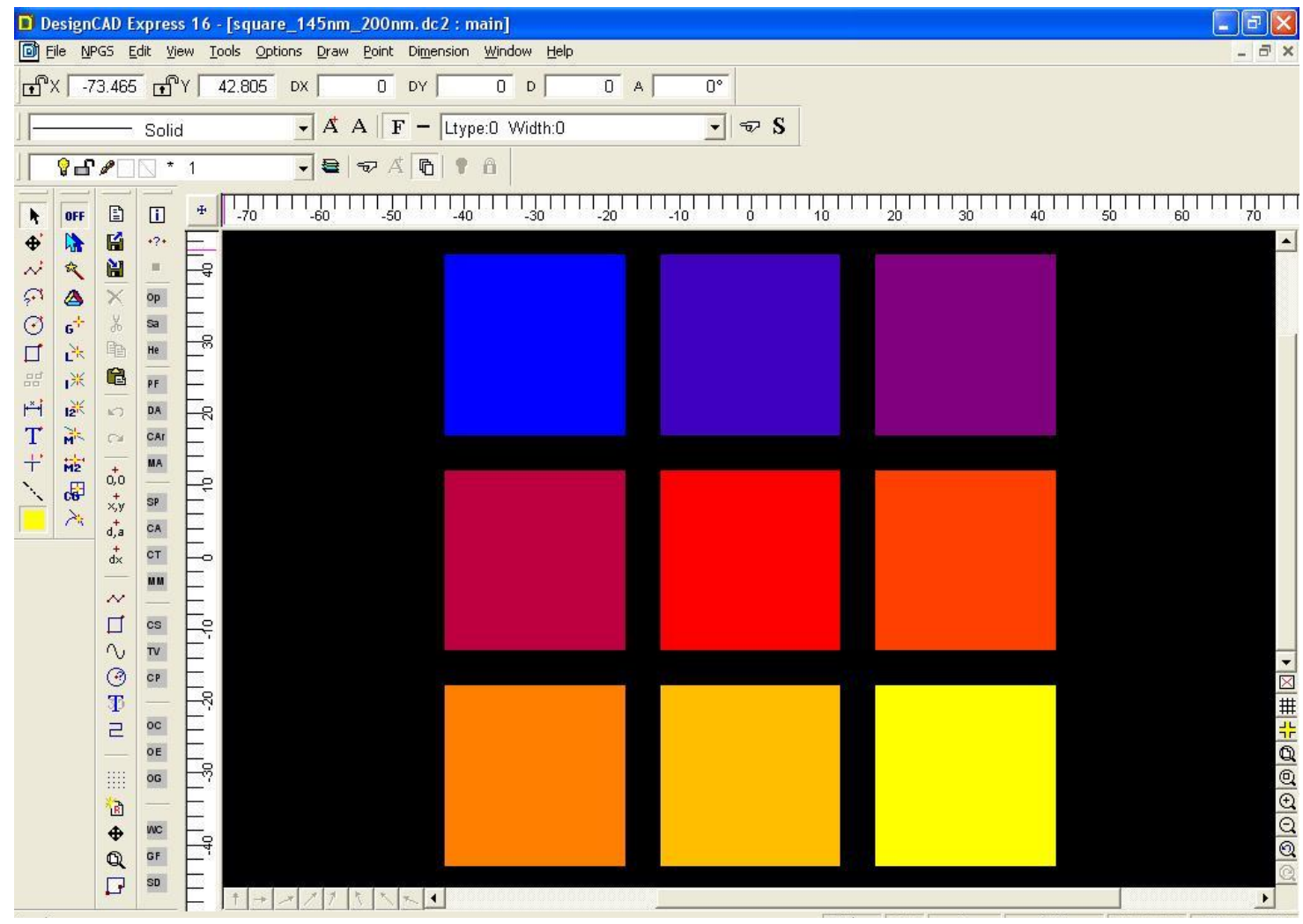

Design CAD file created varying dosages for each nanopattern of size $20 \times 20 \mu \mathrm{m}$ 


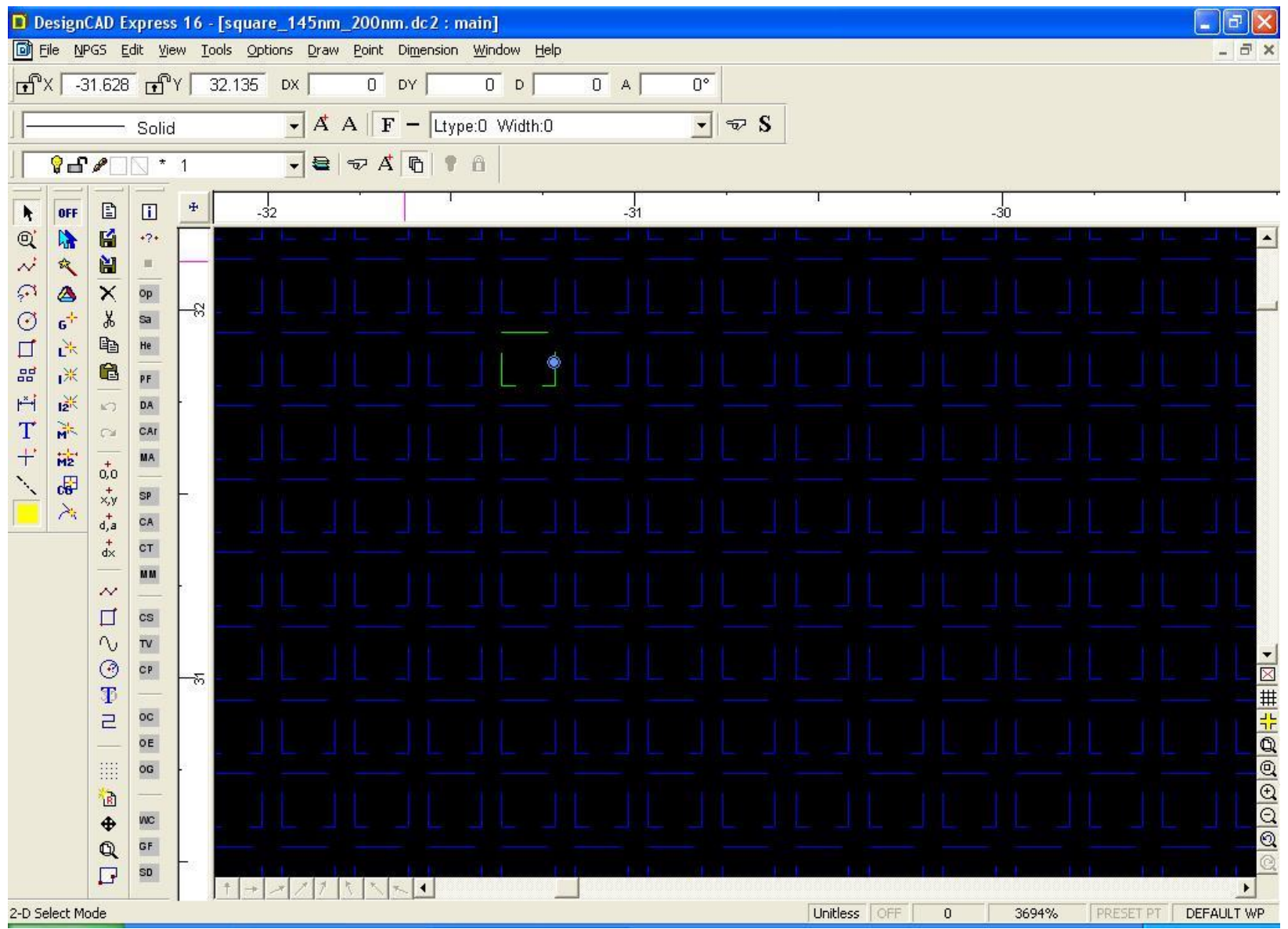

Square nanopillars created by Design CAD file

The Figure shows the square nanopillars in square lattice. 


\section{GOLD STANDARD}

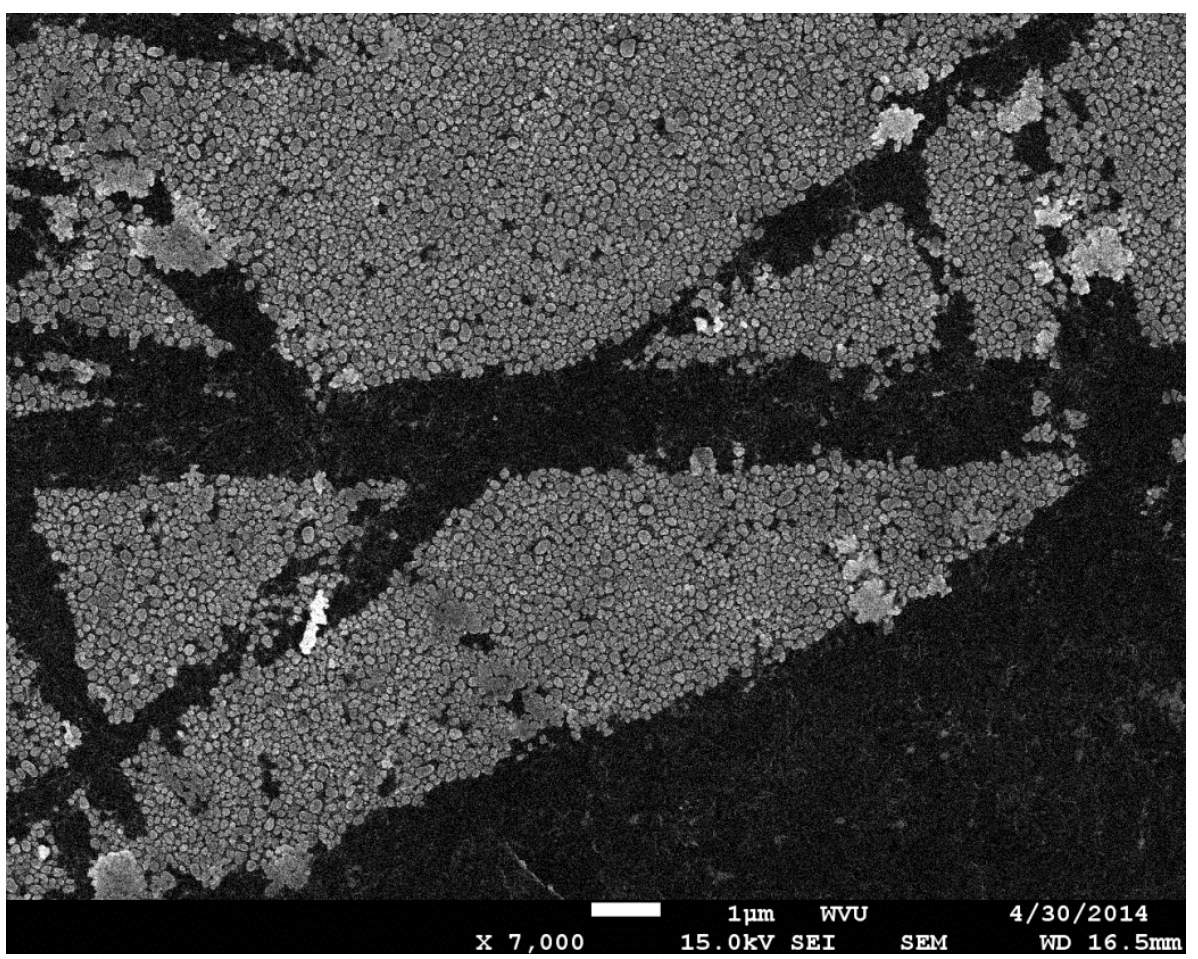

Gold standard showing nano gold particles, imaged at magnification of $7000 \mathrm{X}$

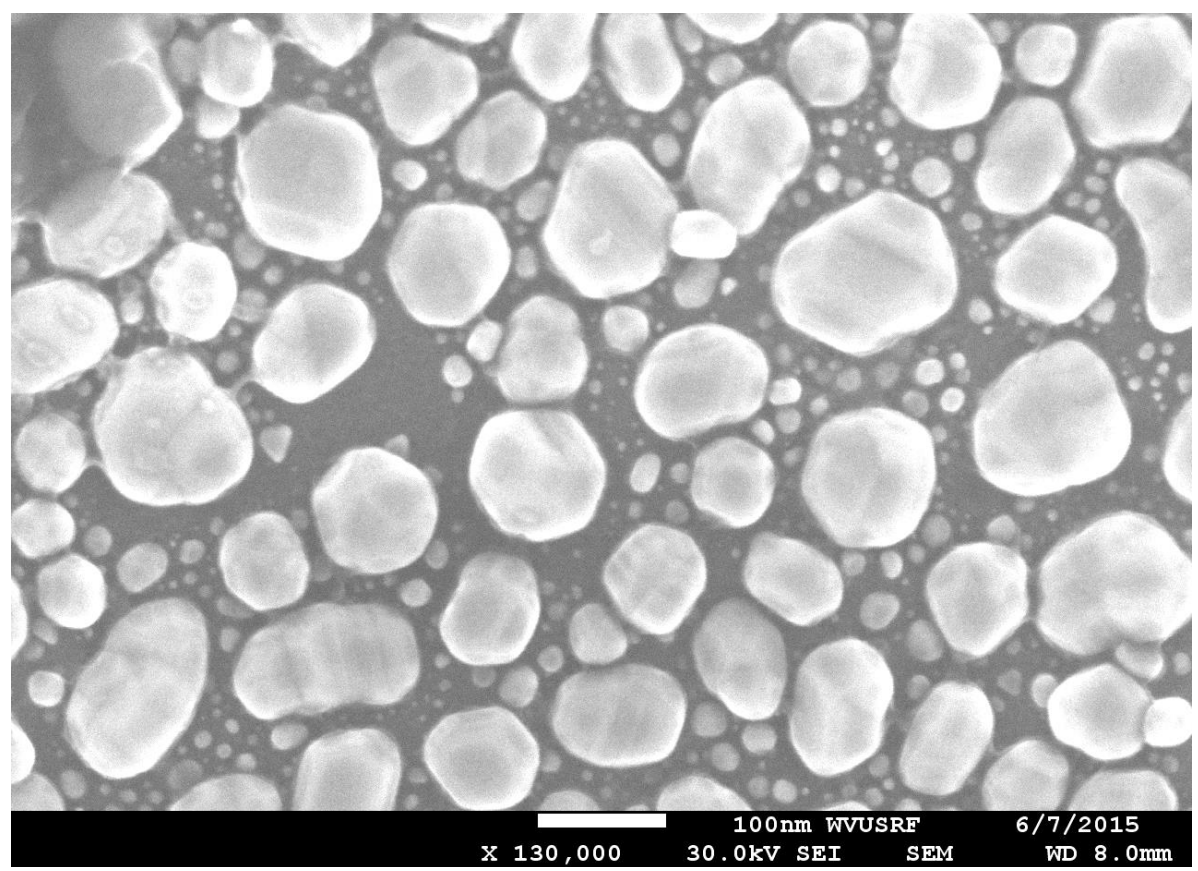

Gold nanoparticles imaged at maginification of 130X 


\section{APPENDIX B: LUMERICAL}

The goal of the simulation is to generate a nanopattern with highest electric field ehnacement arounf the nanostructures at 550nm. FDTD simulation allows to simulate wavelength scale photonic devices. It allows to design and optimize in a 3D TCAD environment. Lumerical is a finite difference time domain software. It is a 3D Maxwell solver. FDTD can solve equations in time domain and can analyze the interaction of UV, visible, and IR radiation with complicated nanostructures structures much less than the operating wavelength of the excitation source. The local electric field enhancement can be calculated by creating an electric field analyzation window.

Lumerical consists of an inbuilt fully vectorial 3D/ 2D computational engine to solve the Maxwell equations.

\section{Simulation setup:}

\section{Structure building}




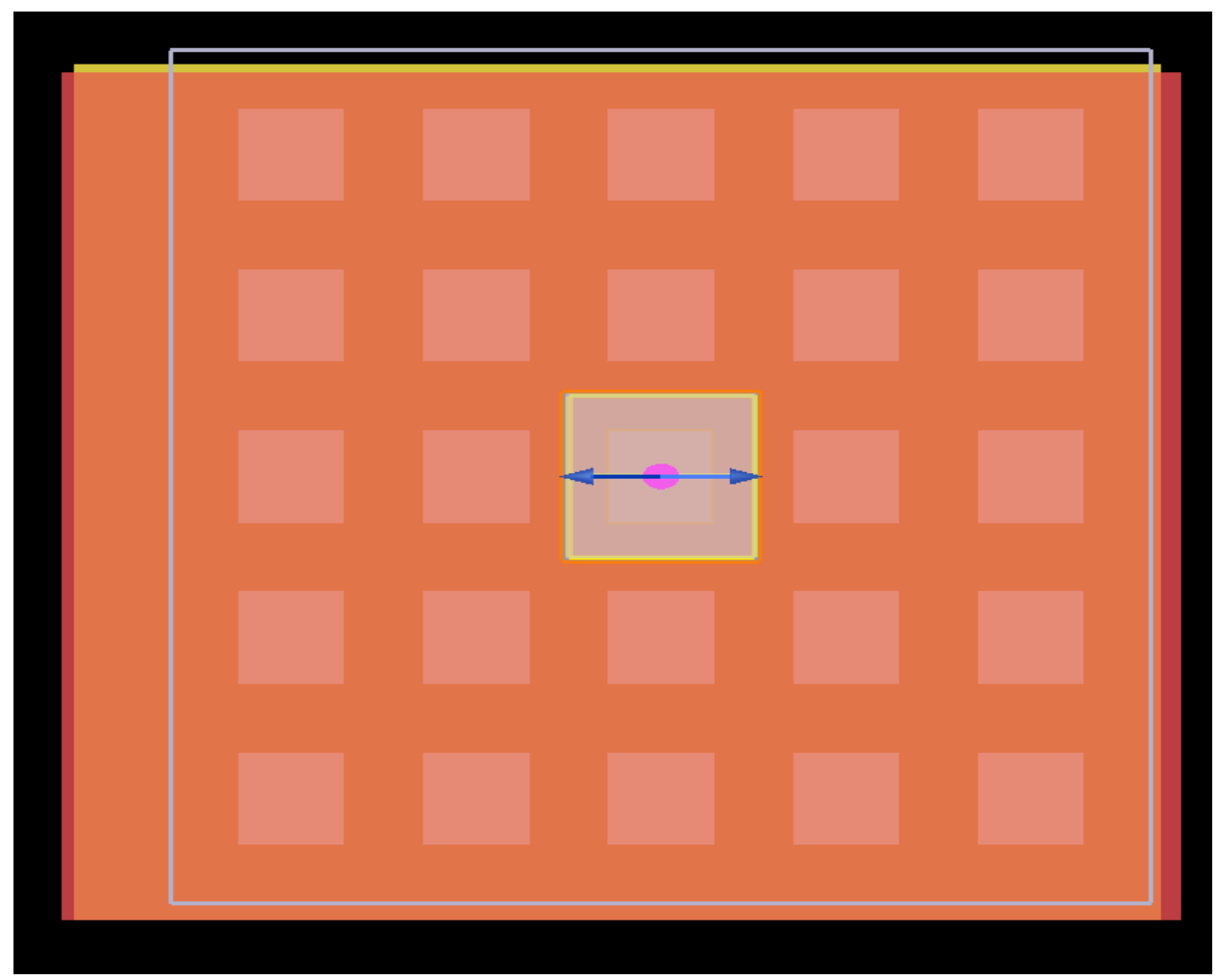

Simulated plasmonic structure using lumerical

The plasmonic nanostructure is constructed using gold nanostructures. Using Si Substrate, over which $\mathrm{SiO} 2$ formed a square lattice structure. with width of $115 \mathrm{~nm}$ and the lattice constant of $200 \mathrm{~nm}$. Then assuming the real time gold deposition, gold nanopillars were formed on the top of $\mathrm{SiO} 2$ pillars and also in between the $\mathrm{SiO} 2$ pillars. When we simulate the struucture, we added a slab out of the rectangles to create Si substrate and then square base rectangles were added on the top of substrate to create the square nanopillars with height of $100 \mathrm{~nm}$. The refractive index for the materials were chosen from the material index database. Johnson and Christy model is chosen as material refractive index for gold. A plane wave source with wavelength range of $400 \mathrm{~nm}$ to $700 \mathrm{~nm}$ is used for excitation and the Lumerical is a Nanophotonic FDTD solver. In built material modellin is available. Extinction crossection were measured. 3D structure is formed by having a silicon substrate, then siO2 pillars on top of it, arranged in square lattice and 
gold square nanopillars on top of it. Then the simulation, sources and monitors are added. Movie monitor provides the time domain response of the fields The electric field intensity at the surface of the gold nanopillars are detected using field monitor

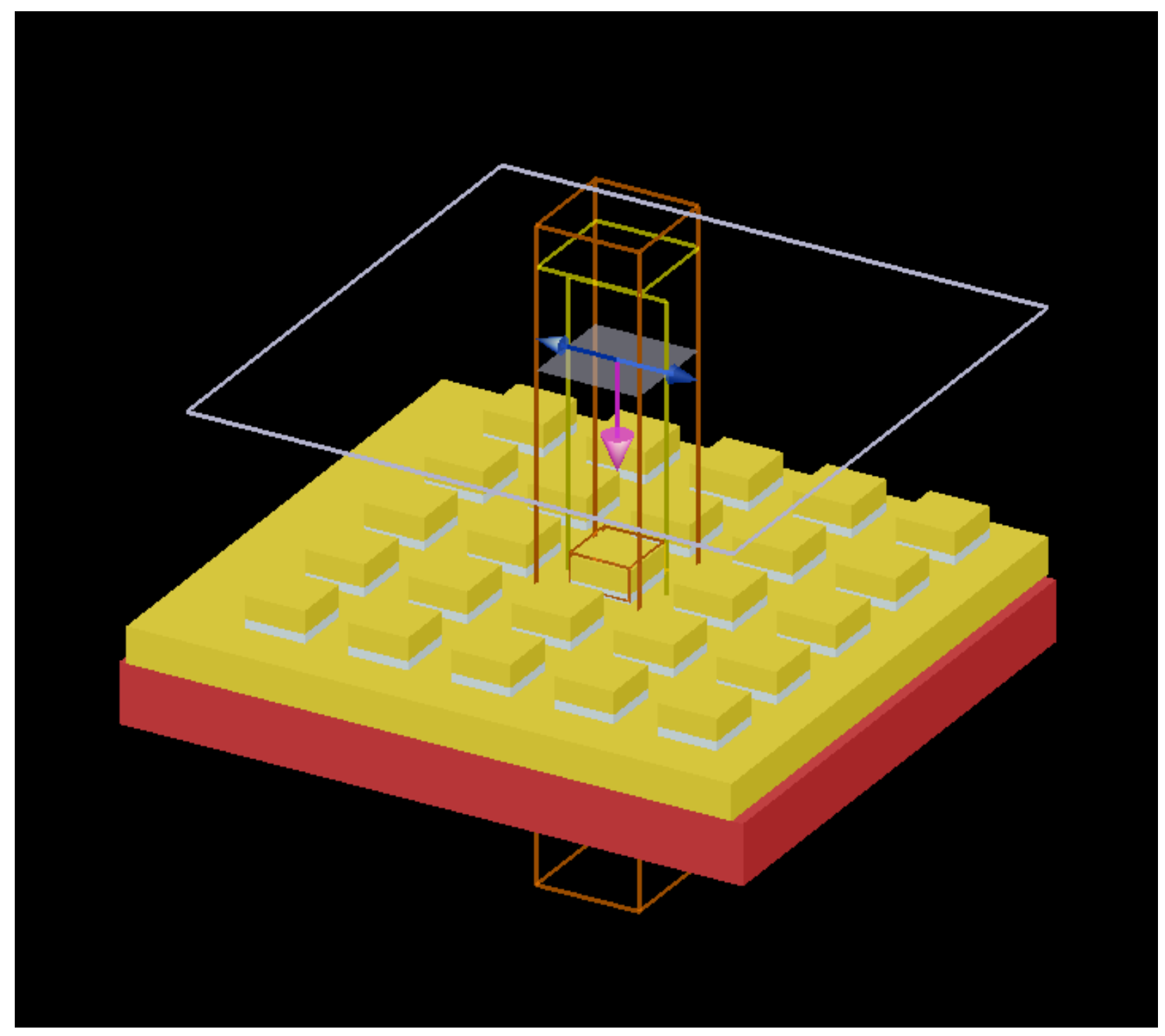

Simulated structure showing structure and analysis windows and sources

The plain wavelength of light is used. Parameter sweeps were ran to calculate the response varying gold deposition thickness, distance between the pillars, and the refractive index. The sweeps were ran and optimized to get the optimized values. 


\section{Material Modeling}

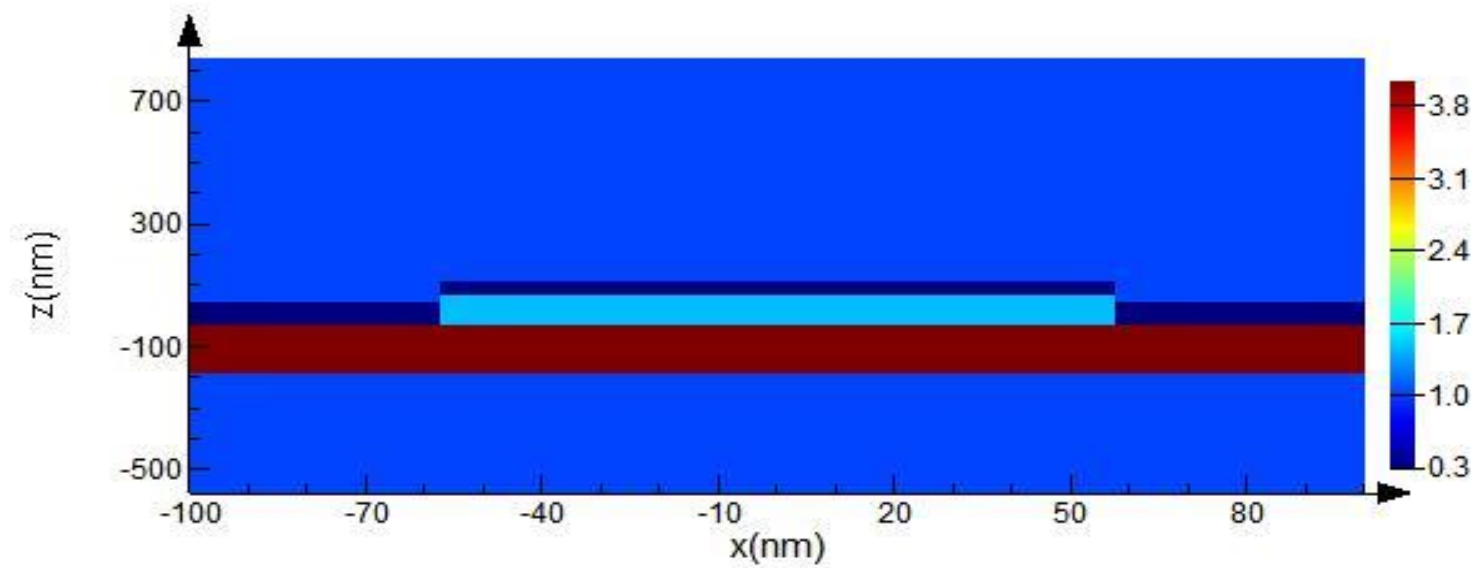

Refractive indices of the sample fabricated

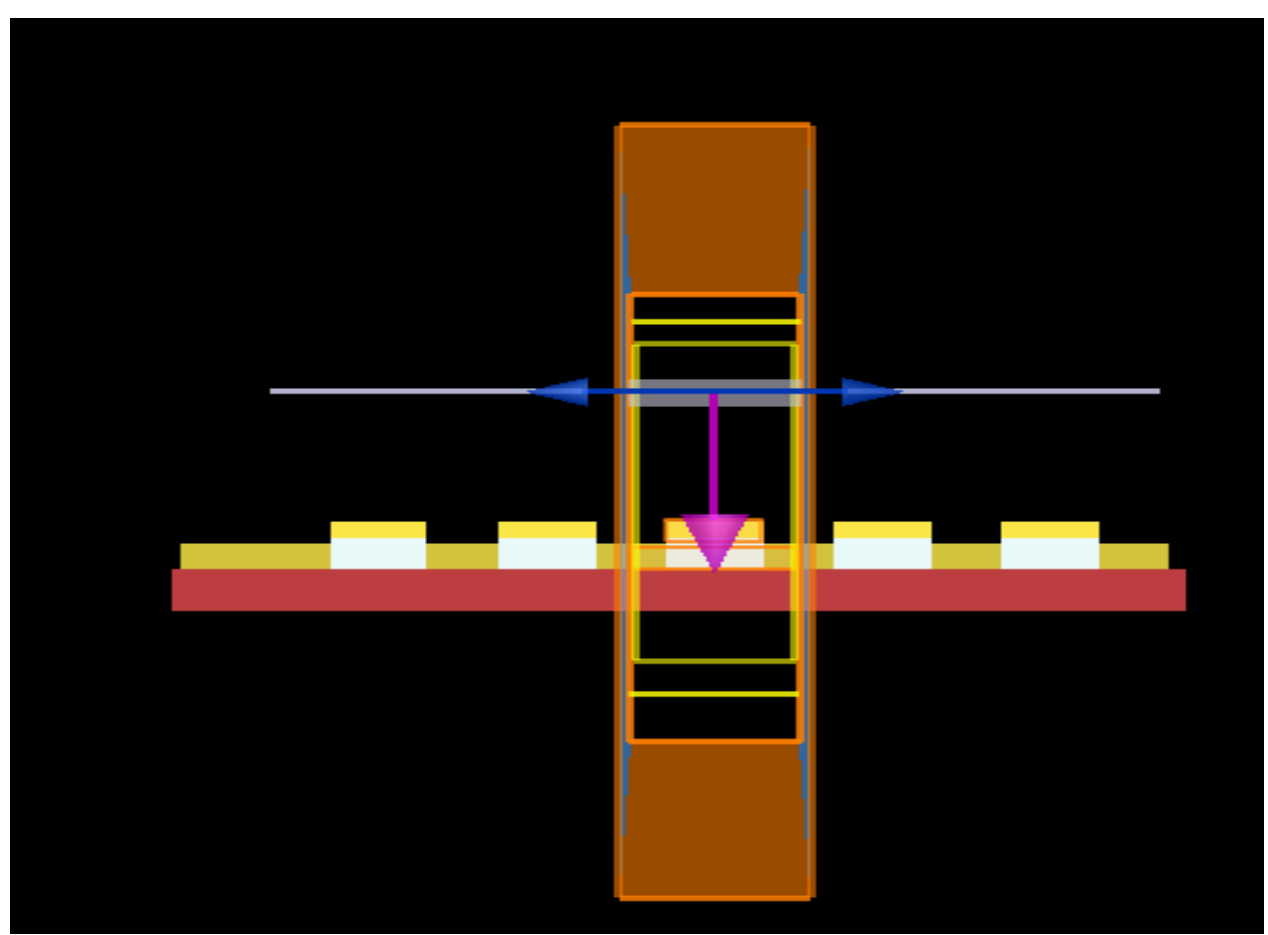

Side view of the simulated structure 
Lumerical consist of inbuilt material refractive index data files for the wide range of wavelengths.

\title{
Boundary conditions
}

The orange boundaries which can be seen in the screenshot above are Perfectly Matched Layer (PML) boundary conditions. PML boundaries absorb incident radiation, and are intended to absorb all radiation propagating away from the cavity. It is important to leave some distance between the cavity and the PML boundaries. If the boundaries are too close to the cavity, they will start to absorb the non-propagating local evanescent fields that exist within the cavity. A simple rule is to leave at least half a wavelength of distance above and below the structure.

Next, notice that the lower half of the simulation $(z<0)$ is shaded blue. This is because we used a symmetric boundary condition on the $\mathrm{z}$ min boundary in order to reduce the computation time by a factor of two. The drawback of using the symmetric boundary condition is that it will forbid certain modes from appearing in the results (modes that do not exhibit the same symmetry relation as the boundary condition). For this PC cavity, there is a plane of symmetry through the center of the slab ( $\mathrm{z}=0$ plane). Using a symmetric boundary condition on this plane will only allow TE-like modes and eliminate TM-like modes from the results.

\section{Sources}

Lumerical supports a large number of souces example: plane wave, dipole, Total field scattered field, wave guide mode.

\section{Scripting}

\author{
Absorption power scripting: \\ f=getdata("Rx","f"); \\ lambda=c/f; \\ t_bottom $=$ transmission $\left(' T x^{\prime}\right)$; \\ t_top = -transmission('Rx'); \\ t_bottom_no = 1-t_bottom - t_top ;
}


plot (lambda,t_bottom_no,'lambda','Absorbed Power','Absorbed Power');

matlabsave("plain",lambda,t_bottom_no);

\section{Electric field calculation script}

$\mathrm{f}=\operatorname{getdata}(" \mathrm{R} "$, "f");

lambda $=\mathrm{c} / \mathrm{f}$;

$\mathrm{T}=$-transmission("T");

$\mathrm{R}=$ transmission $(" \mathrm{R} ")$;

plot(lambda*1e9,T,"wavelength (nm)","NORMALIZED EXTINCTION");

legend("Normalized extinction");

$\mathrm{r}=100 \mathrm{e}-9$;

period $=400 \mathrm{e}-9$;

look at the field profiles recorded at $550 \mathrm{~nm}$, reflected surface

E2_r=getelectric("profile_at_675nm_R");

E2_t $=$ getelectric("profile_at_675nm_T");

E2_xz = getelectric("profile_at_675nm_xz_plane");

$\mathrm{x}=$ getdata("profile_at_675nm_R","x");

$\mathrm{y}=$ getdata("profile_at_675nm_R","y");

$\mathrm{z}=\operatorname{getdata}($ "profile_at_675nm_xz_plane","z");

image(x*1e6,y*1e6,E2_r,"x (microns)","y (microns)","|E|^2 at reflected surface");

image(x*1e6,y*1e6,E2_t,"x (microns)","y (microns)","|E|^2 at transmitted surface");

image(x*1e6,z*1e6,E2_xz,"x (microns)","z (microns)","|E|^2 at y=0");

setplot("colorbar min",0);

setplot("colorbar max",10);

\section{Parameter sweep script}

\#runsweep; 
r=(getsweepdata("Au Thickness","Au_pattern"));

k=(getsweepdata("Au Thickness","Au"));

f=getsweepdata("Au Thickness","f");

tx=getsweepdata("Au Thickness","T");

rx=-getsweepdata("Au Thickness","R");

$\mathrm{f}=\operatorname{getdata}(" \mathrm{Tx} "$, "f");

lambda=c/f;

t_bottom_norm = 1 - tx - rx;

\# plot normalized transmission

plot(lambda,t_bottom_norm,'lambda','normalized power','Absorbed Power');

legend("45","50","55","60","65","70", "75","80","85"); 


\section{APPENDIX C: MATLAB program for image analysis}

Select the folder where the image is present

Run the following code,

$\mathrm{X}=$ Imread('Test1.png');

imshow(X);

These commands will open the image and using the data cursor tool in the tool box, find the RGB pixel values 